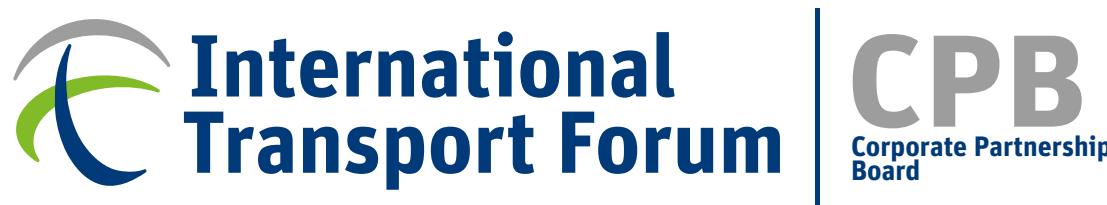

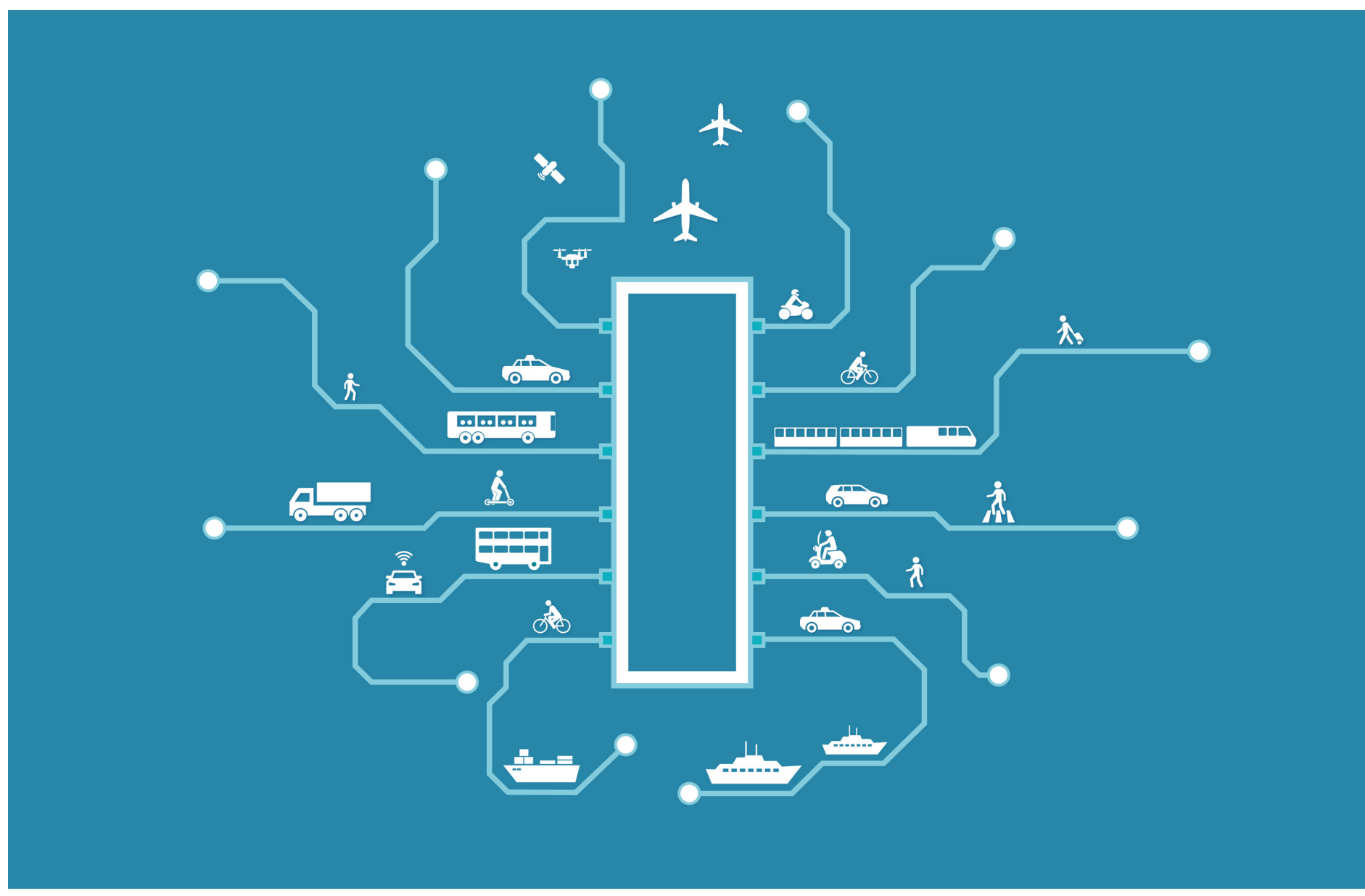

\section{New Directions}

for Data-Driven

Transport Safety

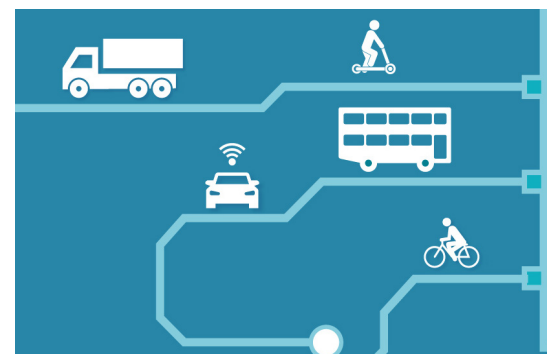

Corporate Partnership Board Report 


\section{New Directions \\ for Data-Driven Transport Safety}

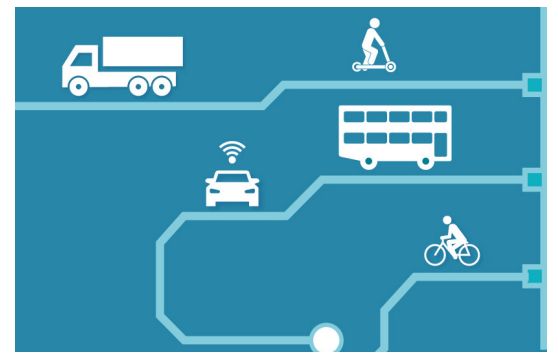

Corporate Partnership Board Report 


\section{About the International Transport Forum}

The International Transport Forum at the OECD is an intergovernmental organisation with 59 member countries. It acts as a think tank for transport policy and organises the Annual Summit of transport ministers. ITF is the only global body that covers all transport modes. It is administratively integrated with the OECD, yet politically autonomous.

ITF works for transport policies that improve peoples' lives. Our mission is to foster a deeper understanding of the role of transport in economic growth, environmental sustainability and social inclusion and to raise the public profile of transport policy.

ITF organises global dialogue for better transport. We act as a platform for discussion and pre-negotiation of policy issues across all transport modes. We analyse trends, share knowledge and promote exchange among transport decision makers and civil society. ITF's Annual Summit is the world's largest gathering of transport ministers and the leading global platform for dialogue on transport policy.

Our member countries are: Albania, Argentina, Armenia, Australia, Austria, Azerbaijan, Belarus, Belgium, Bosnia and Herzegovina, Bulgaria, Canada, Chile, China (People's Republic of), Croatia, Czech Republic, Denmark, Estonia, Finland, France, Georgia, Germany, Greece, Hungary, Iceland, India, Ireland, Israel, Italy, Japan, Kazakhstan, Korea, Latvia, Liechtenstein, Lithuania, Luxembourg, Malta, Mexico, Republic of Moldova, Montenegro, Morocco, Netherlands, New Zealand, North Macedonia, Norway, Poland, Portugal, Romania, Russian Federation, Serbia, Slovak Republic, Slovenia, Spain, Sweden, Switzerland, Turkey, Ukraine, the United Arab Emirates, the United Kingdom and the United States.

\section{About the Corporate Partnership Board}

The Corporate Partnership Board (CPB) is the International Transport Forum's platform for engaging with the private sector and enriching global transport policy discussion with a business perspective. The members of the ITF Corporate Partnership Board are: Abertis, AB InBev, Alstom, Aramco, Bird, Bosch, Brisa, ExxonMobil, Incheon International Airport, Kakao Mobility, Kapsch TrafficCom, Latvian Railways, Michelin, North Adriatic Sea Port Authority, NXP, PTV Group, RATP Group, The Renault-Nissan-Mitsubishi Alliance, SAS, Siemens, SNCF, Total, Toyota, Uber, Valeo, Volvo Cars, Volvo Group and Waymo.

\section{Disclaimer}

Funding for this work has been provided by the ITF Corporate Partnership Board. This report is published under the responsibility of the Secretary-General of the ITF. It has not been subject to the scrutiny of ITF or OECD member countries, and does not necessarily reflect their official views or those of the members of the Corporate Partnership Board. 


\section{Acknowledgements}

The work for this report was carried out in the context of a project initiated and funded by the International Transport Forum's Corporate Partnership Board (CPB). CPB projects are designed to enrich policy discussion with a business perspective. They are launched in areas where CPB member companies identify an emerging issue in transport policy or an innovation challenge to the transport system. Led by the ITF, work is carried out in a collaborative fashion in working groups consisting of CPB member companies, external experts and ITF staff.

Many thanks to the members of the Corporate Partnership Board companies involved in this work: Abertis, ABInBev, Alstom, Bosch, Brisa, ExxonMobil, Incheon International Airport Corporation, Kapsch TrafficCom, Latvian Railways, Michelin, NXP, PTV Group, RATP Group, Renault Nissan Mitsubishi Alliance, SAS Institute, Siemens, SNCF, Total, Toyota, TransDev, Uber, Valeo, Waymo.

The report draws conclusions from the ITF CPB Workshop "New directions for data-driven transport safety" held 3-4 December 2018 at the OECD in Boulogne, France. Participants of the workshop included:

Jean Marc Beteille, RATP Group

Joao Bernardino, Transportes Inovação e

Sistemas

Jonathan Bibas, Ellis-Car

Jean-Baptiste Burtscher, Valeo

Maguelonne Chandesris, SNCF

Philippe Crist, International Transport Forum (ITF)

Véronique Feypell, ITF

Rahul Goel, University of Cambridge

Karsten Kremer, PTV

Takayuki Kusajima, Toyota

Vincent Ledoux, Cerema

Manuel Lianos, NXP

Darren Lindsey, Michelin

Sharon Masterson, ITF
Mollie McArdle, Open Transport Partnership and SharedStreets

Laura Meynier, ITF

Luis Miranda-Moreno, Brisk Synergies, McGill University

Rachele Poggi, ITF

Marc Ribo, Abertis

David Roiné, Valeo

Manuelle Salathé, Observatoire National Interministériel de sécurité routière

Sofia Salek de Braun, PTV

Alexandre Santacreu, ITF

Neil Thorpe, Newcastle University

Philippe Girard, Total

Philippe Ventejol, RATP Group

George Yannis, National Technical University of Athens

Special thanks go to Annie Chang (SAE), Ieva Kustova (Latvian Railways), Jasja Tijink (Kapsch TrafficCom), Sam Chapman (The Floow), Jane Lupson (Transport for London) and Brendan Halleman (IRF Global) for contributions and insightful comments. At the International Transport Forum, credits go to Sokob Challener and Hilary Gaboriau for support and editing, to Veronique Feypell and Rachele Poggi for their contributions to the workshop, to Katja Schechtner and Gyuhyung Lee for support on drones and aviation.

The principal authors of this report are Alexandre Santacreu, Laura Meynier and Philippe Crist. The project was managed by Philippe Crist and Alexandre Santacreu of the International Transport Forum. Coordination of CPB activities was carried out by Sharon Masterson. 


\section{Table of contents}

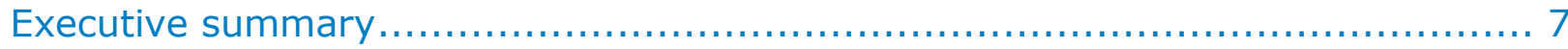

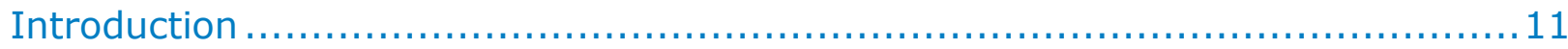

Safe System and Vision Zero: The role of data $\ldots \ldots \ldots \ldots \ldots \ldots \ldots \ldots \ldots \ldots \ldots \ldots \ldots \ldots \ldots$

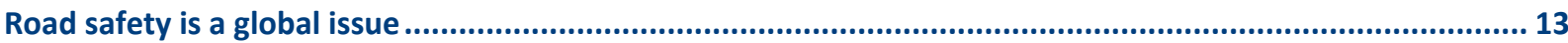

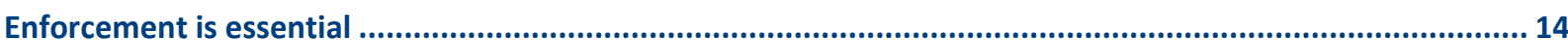

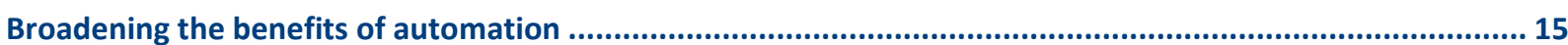

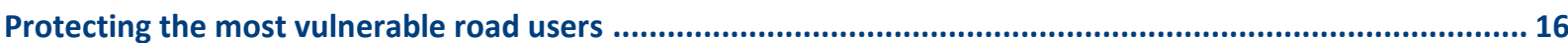

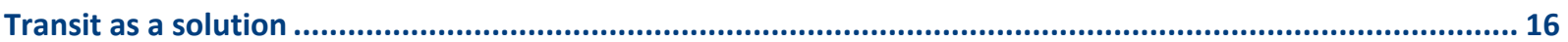

Gathering intelligence on crashes and injuries ..................................... 18

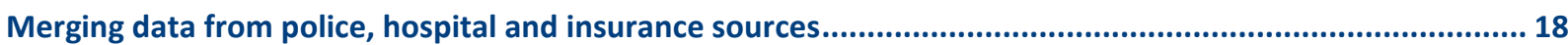

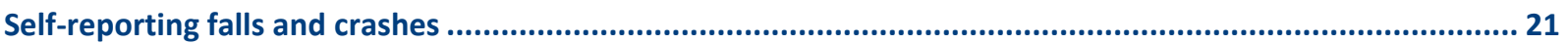

In-depth crash investigation using Event Data Recorders .................................................................................. 24

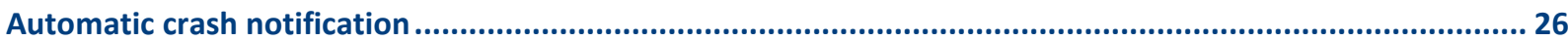

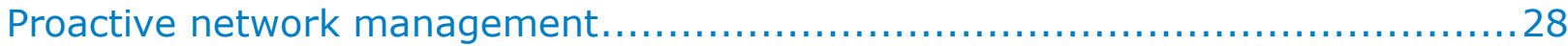

Surrogate safety metrics to power crash prediction models .............................................................................. 28

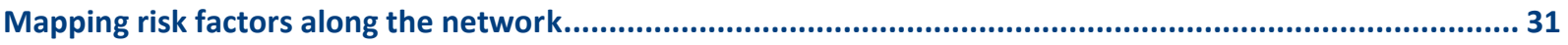

How to map and monitor speeding................................................................................................................... 33

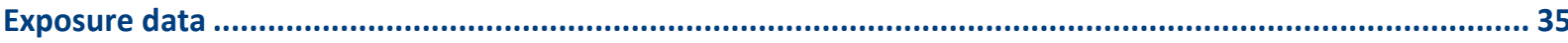

Infrastructure inspection and predictive maintenance through mobile sensors and imagery ......................... 36

A growing role for drones and satellites .......................................................................................................... 39

User feedback on infrastructure faults and incidents ...................................................................................... 41

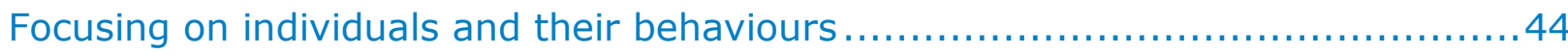

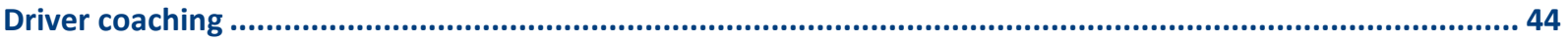

Sensing driver distraction and drowsiness ..................................................................................................... 45

Monitoring the behaviour of professional drivers ............................................................................................... 47

Insurance telematics and their impact on behaviour ................................................................................... 51

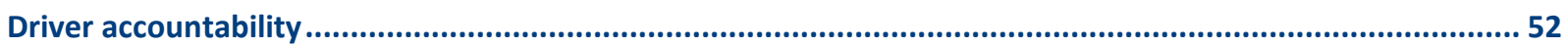


Expanding Cooperative Intelligent Transport Systems ............................... 55

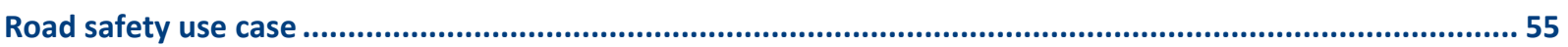

Competing solutions for short range communication...................................................................................... 57

Protecting vulnerable road users with cooperative systems ................................................................................ 58

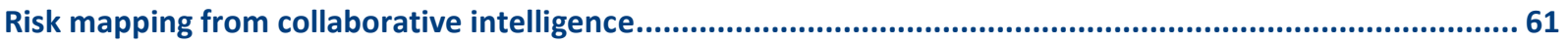

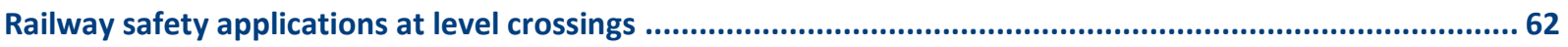

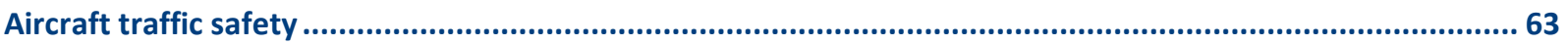

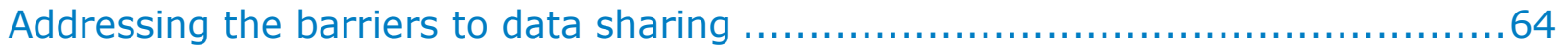

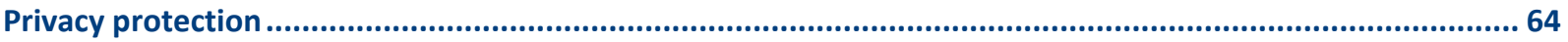

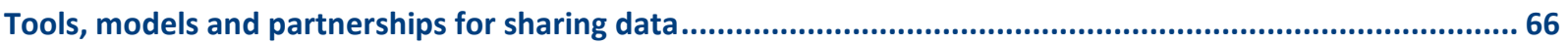

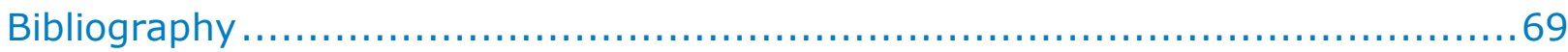

\section{Tables}

Table 1. Comparing the traditional road safety approach with a Safe System ............................ 13

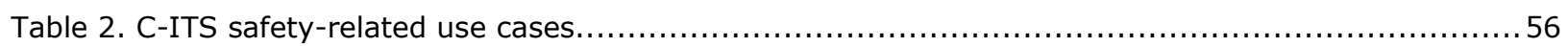

\section{Figures}

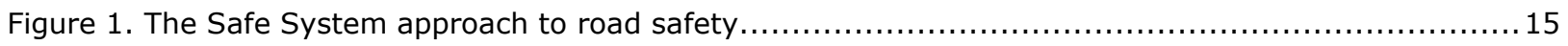

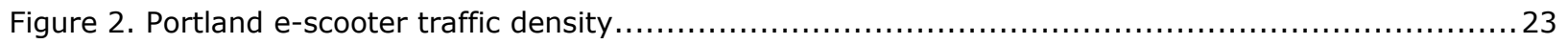

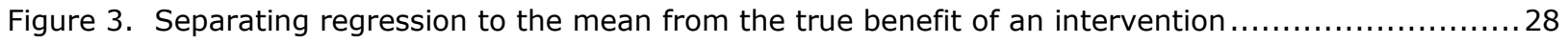

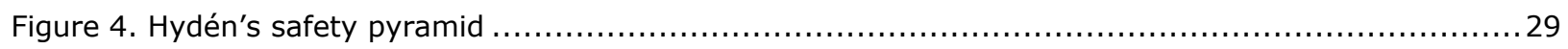

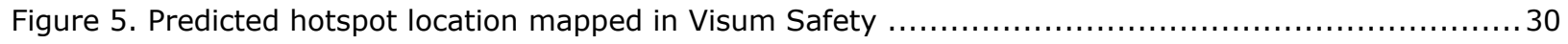

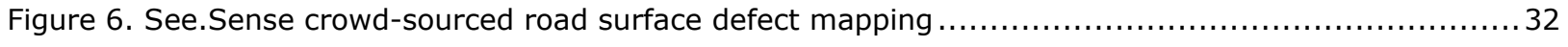

Figure 7. Passenger pick-up and drop-off locations provided by ride-sourcing companies...................... 33

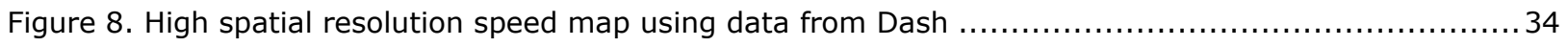

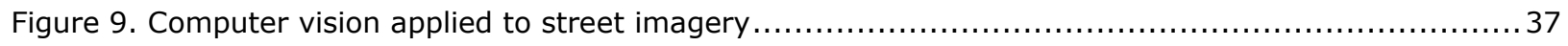

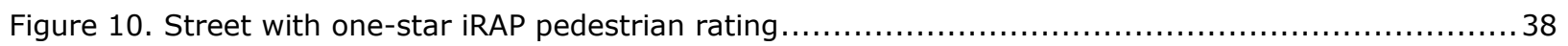

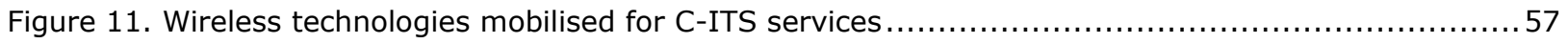

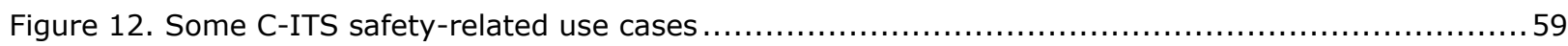

Figure 13. Dashboard of a surface movement control system at Incheon International Airport................63

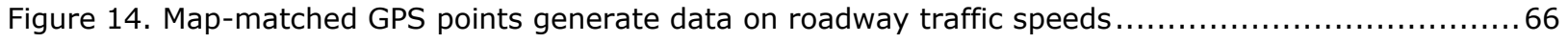

\section{Boxes}

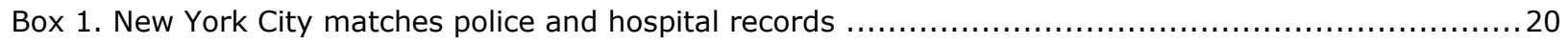

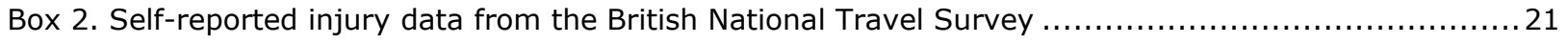




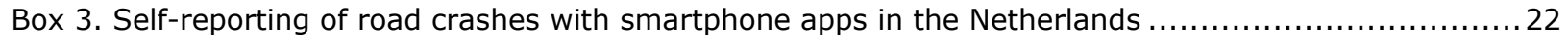

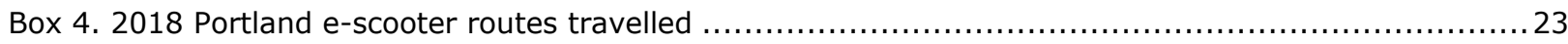

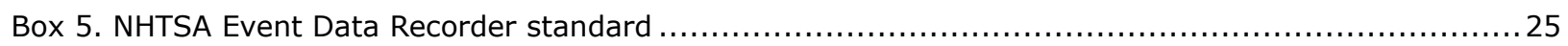

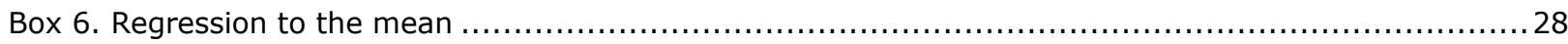

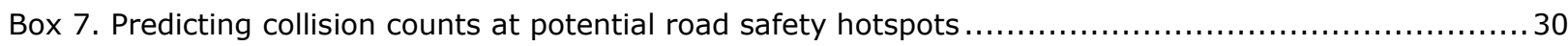

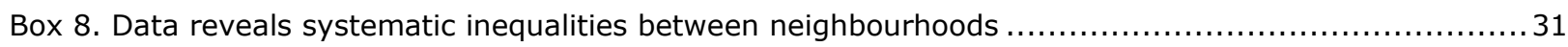

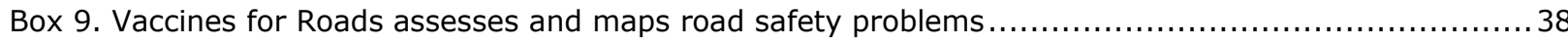

Box 10. Safety for rail transport: Siemens Mobility and Strukton Rail's Video Track Inspector..................38

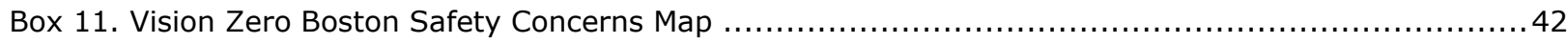

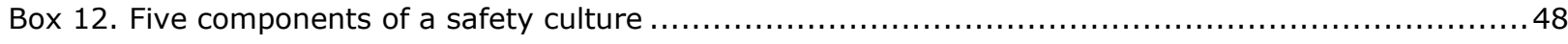

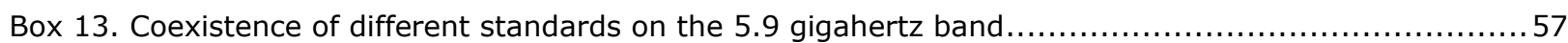

Box 14. European projects PROSPECT and XCYCLE develop innovative solutions to protect pedestrians

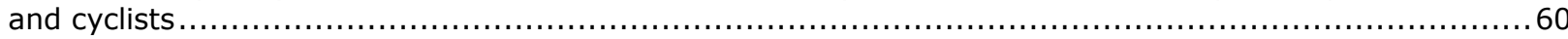

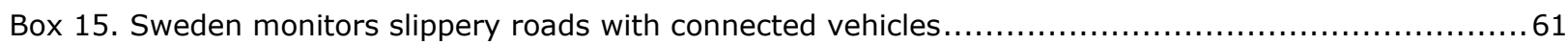

Box 16. Latvian Railway and Datakom develop smart safety measures for level crossings................62

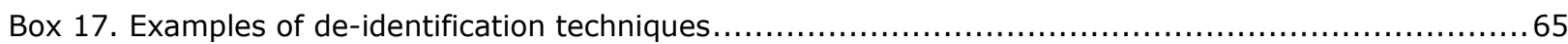

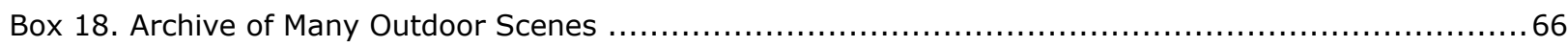

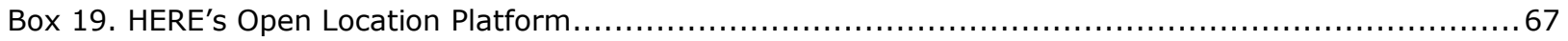




\section{Executive summary}

\section{What we did}

This report explores how seamless data collection, analysis and sharing can unlock innovations in transport safety. The rise of smartphones, sensors and connected objects is relentlessly producing more data. Progress in computing power, data science and artificial intelligence make it possible to interpret them. This presents an opportunity for the transport sector in general and for transport safety in particular. This report thus focuses on road safety, drawing on experiences from all transport modes. It examines emerging solutions to better assess crash numbers and to understand factors that contribute to them. It also identifies ways to address infrastructure safety problems in a proactive manner and to tackle road user errors. Finally, the report explores how road safety can benefit from driver assistance systems and cooperative connected solutions. The focus here is on the role of data sharing, including such important aspects as privacy protection, data standards and the role of partnerships. The work builds on the findings of a workshop held in December 2018 and on input from numerous experts.

\section{What we found}

The majority of countries lack reliable, complete and comparable data on road crashes and associated injuries. Often only the number of fatalities is known. Information technology can help address this gap, including through solutions that involve hospital data or self-reporting. New data sources also offer many opportunities for understanding crash risks.

In the rail sector, a predictive maintenance model is already helping to prevent safety-critical failures on tracks and trains. Along the road network, automatic data collection is also possible thanks to instrumented vehicles and smartphones reporting information on factors including hard braking, poor road surfaces and excess speed. Surrogate safety metrics could be developed using data on the engagement of active safety systems. These include anti-lock braking systems (ABS), electronic stability control (ESP) and autonomous emergency braking (AEB). The activation of such systems provides some of the simplest yet most relevant surrogate safety data a vehicle can produce.

The concept of surrogate safety metrics is increasingly used to diagnose traffic safety problems and take action before a serious crash happens. Surrogate safety metrics are based on identifying the occurrence and severity of traffic conflicts involving evasive actions such as braking or swerving, but not necessarily collisions. Using artificial intelligence, large amounts of video footage and data points from road-side cameras and sensors can be analysed to identify close calls in which a crash was narrowly avoided.

Street imagery can be used to support the assessment of the safety characteristics of roads. This already happens for the attribution of star ratings under road assessment programmes. The next generation of star-rating programmes will most likely benefit from more frequent and broader collection of image data as well as automated image analysis from computer vision solutions. Drones and satellites can capture additional data and will play an increasing role for road safety. These innovations will facilitate the planning of road safety investments as well as the monitoring of results.

Cooperative Intelligent Transport Systems (C-ITS) will enable connected vehicles to openly broadcast relevant warnings. In addition to communicating with each other and with the road infrastructure, connected vehicles will be able to report on system performance in real time and throughout the entire road network. This should benefit all road users, connected or not. Network managers could use data from short range messages as a rich source of information, where road-side receivers are installed. 
Sharing vehicle-related data in a common format could enhance road safety. Technologies like automatic crash notification and event data recorders already propose data-driven systems for emergency response and crash investigations.

A host of telematics solutions exist for fleet management, usage-based insurance, eco-driving and safe driving coaching. Smartphones are becoming increasingly capable of running such applications and the flexibility they offer could make such solutions increasingly popular. Their market penetration, processing power and communication capability make them an obvious choice for transport safety-related data collection. To complement automated data collection, transport users and workers can use smartphones to voluntarily report close calls, infrastructure defects or unsafe behaviour, often in real-time.

New technologies offer solutions but also pose problems. Big data is prone to many of the same errors and biases that plague smaller data sets. Thus, more data is not helpful in itself; it only provides better answers if analysed with rigor. Big data also creates privacy threats, especially with the growing risk of reidentification of individuals in anonymised data sets.

Information technology can also have negative impacts on road safety. For instance, drivers might evade speed enforcement using dedicated alert systems. They could also be distracted from driving by new services, from navigation and infotainment to Cooperative Intelligent Transport System (C-ITS) alerts and coaching. And, conscious of the protection offered by active safety systems, drivers may take their eyes away from the road over sustained periods of time. Generally, driver distraction is likely to increase as smartphones, smart glasses and vehicle infotainment systems compete for the driver's attention. There will be little commercial incentive to shut down such systems during driving, as users will want to be entertained during an increasingly automated, hence uneventful, drive.

\section{What we recommend}

\section{Develop standards and platforms for the collection and sharing of safety-critical vehicle data}

Requirements for the collection and sharing of data should be imposed on shared vehicles, whether cars, bicycles or scooters, whether standard or electric. An international data standard would help operators to launch services in new places. It would help public authorities to monitor whether services deliver on policy targets. The data could include geo-located information about falls, crashes, and the engagement of safety systems. Data on start and end points, turns, spot speed, pavement riding and wrong-way riding should also be registered, as should traffic volumes. Standard data reporting formats should be jointly established by the industry and regulators to facilitate processing and analysis. Excessive regulatory pressure on emerging mobility services in comparison to traditional services should, however, be avoided.

\section{Ensure privacy in the use of safety-critical data}

The use of data to improve safety outcomes should not erode privacy or the protection of personal data. Data collection and analysis should be fully in line with existing privacy frameworks and should evolve as those frameworks themselves evolve. A wide range of privacy solutions exist to increase use of data for traffic safety and respect the privacy of individual users. Approaches such as de-identification, data swapping, variable sampling or encryption can help ensure the balance between optimising safety and addressing privacy concerns.

\section{Refine the applications of surrogate traffic safety metrics}

Further research should aim to identify which surrogate safety metrics can predict road traffic crash numbers most successfully and which can specifically predict the number of people killed and seriously injured. Research should also explore how surrogate safety metrics could include factors such as the fragility of a crash victim, speed, mass and crash angle. The transport community should work to 
harmonise terms, definitions and techniques for surrogate measures of safety to provide a sound basis for this research.

Harness Big Data for road safety but beware of biases

A lot of excitement surrounds the potential of Big Data. Road safety professionals need to acknowledge a number of pitfalls, however. Every dataset should be considered to be biased towards some user group, trip purpose or other dimension unless proven otherwise. The expertise of qualified data scientists and statisticians will be needed to counter the risk that the opportunistic analysis of low-cost data misleads decision makers.

Review training needs for road safety professionals

The adoption of surrogate safety metrics provides an occasion to review statistical core competencies among road safety professionals and identify training needs. A good understanding of key statistical concepts is essential to prevent the misinterpretation and misuse of data. Strengthening links between transport safety experts and specialised academic researchers could be beneficial.

Empower transport users and workers through mechanisms to report safety concerns

Feedback systems that maximise participation from workers and from the travelling public can provide valuable data on transport safety. Transport workers should have access to a trusted channel for reporting safety issues without fear of recrimination. Passengers should be able to help identify potential dangers, for instance risky behaviour by drivers in the bus and taxi industries. Road users should have a mechanism to submit video evidence of unsafe driving, or photos of road defects. Highway authorities should join forces and provide such platforms, harmonise user experience, share costs and consider open-source solutions.

Make safety-critical vehicle data available for telematics applications

Vehicle telematics could have significant effects on driver behaviour. Solutions increasingly rely on smartphones to detect vehicle dynamics. Establishing a new standard for the wireless dissemination of vehicle information would encourage the use of valuable data. The standard data protocol should allow the monitoring of driving conditions for insurance purposes, coaching or other uses. Hacking risk could be eliminated by designing the standard as a one-way broadcast.

Find ways to integrate smartphones into Cooperative-ITS to benefit all users

Private cars should contribute to data collection as shared vehicle fleets do already. Cooperative ITS technology enables every vehicle to openly and regularly broadcast its position and send warning messages when relevant. For these messages to benefit the wider community, including non-connected vehicles, smartphones should be integrated into the C-ITS system. Policy makers should allocate frequency bands for C-ITS safety application and promote the introduction of interoperable C-ITS systems worldwide.

Improve and link police and hospital data on road crash injuries

Most countries have a poor understanding of the true number of serious injuries and of crash circumstances leading to these injuries. Improving the quality of police and health records is important. Equally important is to link data from transport and health spheres, be it for occasional or permanent linkages. Self-reported traffic injury surveys also have a role to play in complementing other datasets.

Prevent, detect and signal driver distractions

New vehicles should include distraction and drowsiness alerts as a standard feature. These should preferably be based on eye tracking technologies. Crash investigators should have access to eye tracking data through event data recorders ("black boxes"). Smartphone apps developed by the car insurance 
industry could prevent the phone from functioning when activated by a driver, but potentially also monitor eye movements and thus alert on other distractions.

Revise trigger mechanisms for automatic crash notification and event data recording

Vehicle crashes with pedestrians and other vulnerable road users often do not trigger airbags, which in turn would typically trigger an automatic crash notification (such as eCall) and an event data recorder.

Vulnerable road users that become victims of a crash with a vehicle thus rarely benefit from such systems. The trigger mechanism should thus be reconsidered and revised to ensure that all road users benefit from these potentially life-saving alert and investigation systems. The solution could involve sensors or forwardfacing cameras that are already fitted to vehicles as part of autonomous emergency braking systems.

Share data to enforce limits on driving hours in the gig economy

In the gig economy, independent workers are hired as self-employed contactors to carry out short-term assignments. Typical assignments in the gig economy include driving vehicles for ride-sourcing operators and delivering goods and food for platform-based companies. The auto-entrepreneurs typically face high pressure to work long hours to make their activity gainful. To prevent breaches in the driving hours restrictions applicable to other commercial drivers, ride-sourcing and delivery platforms could be required to share data on driving and riding time via the licence numbers of their contractors.

Favour more accurate and relevant geo-spatial accuracy for safety applications

Information about safety-relevant events such as hard-braking can help better target safety-improving interventions. Their geo-spatial accuracy needs to be consistent and detailed, however. Hybrid geo-spatial sensing approaches combining multiple sources should be preferred over single-sensor approaches (like using only smartphone apps) for road safety applications.

Update legal frameworks to account for ubiquitous sensing data and their use in improving safety

Data streams from sensors, like those provided by increasingly ubiquitous video data, can contribute to better safety outcomes if used for analysis. Legal frameworks should be reviewed and adapted to accommodate the collection and use of such sensor data. In line with current data protection policies, only post-incident data should be preserved for legal purposes, and only sufficiently de-identified and aggregated data should be used for measuring safety outcomes and targeting interventions. 


\section{Introduction}

Quantities of data are generated daily in transport from transactions, location services, and sensors (tracking physical activity, for instance). Transport provides an immense opportunity for data to be collected and turned into valuable insights. This report focuses on harnessing newly-available and large, often unstructured data with the aim of improving transport safety.

Safety in road transport lags far behind the rail and aviation sectors, where safety takes precedence over efficiency or convenience, and where risks are addressed at system-level rather than at user-level. Train drivers and aircraft pilots, for example, are rarely given the responsibility of determining speeds and headways they wish to adopt.

While rail and aviation systems are based on a zero tolerance policy and have relatively low per kilometre death rates, road traffic results in an intolerable 1.35 million deaths per year - a toll that continues to rise. Road crashes have become the leading cause of death in children aged between five and 14, but also in young people aged between 15 and 29 (WHO, 2018).

In response to this, a number of countries have embraced the Safe System approach to transport safety. This approach treats crash-related deaths and serious injuries as largely preventable and not as an inevitable price to pay for the mobility of people and goods. This paradigm shift is gaining traction at a time when technology is creating opportunities for data-driven solutions to support the new approach.

Current trends are shaping a future with ubiquitous connectivity. Smartphone penetration is near $80 \%$ in almost every developed country (Deloitte, 2017), and more than three billion people use mobile internet globally (GSMA, 2018). The number of Internet of Things (IoT) connections will increase more than threefold between 2017 and 2025, reaching 25 billion, driven by a proliferation of use cases for smart homes and smart cities.

Location services built on location-sensing technologies and data from other location-relevant signals, such as Wi-Fi positioning, are widely adopted by members of the public, not least for navigation purposes, but also to identify nearby transport options and services. Beyond smartphones, location technologies are also embedded in vehicles and wearable technologies such as health monitors. Location services are becoming more accurate as the world becomes more connected, as every fixed connection serves as a location beacon. Their accuracy is complemented by a growing number of novel location-sensing technologies and new global satellite positioning systems.

Sensors are increasingly embedded into mobile devices or vehicles. Facial scanners, embarked LIDAR (light detection and ranging) sensors, radars and cameras, combined with adequate processing power and adapted algorithms, are able to recognise users and make sense of their environment. Sophisticated massproduced sensors are now on wearable devices and transport modes like shared bicycles and e-scooters.

Sensors on vehicles provide new opportunities for enhanced safety (ITF, 2015). They provide vehiclerelated data that, if shared in a common format, could enhance road safety. Work is underway to harmonise standards regarding data sharing protocols in order to accelerate safety improvements and lower implementation costs for conventional and increasingly automated vehicles.

As the connected network of objects expands, it generates an amount of data which is growing at an exponential rate, creating opportunities and raising new challenges. Considerable uncertainty remains as to if and how data will contribute to a different approach to transport safety. Can we learn precious insights from the collected data, and use it to improve safety outcomes? If so, do we have the capacity to access, process (either on-board or remotely) and make sense of so much information? What might be the effects on other sectors? For example, will the insurance sector be transformed by the use of behaviour-tracking 
technologies in smartphones and other devices to provide nudges for people to adopt safer behaviours? Will data collection infringe on essential privacy protection principles and provoke public backlash which would harm a range of safety use cases? Are adequate policies in place to guide the public and private exploitation of this data along the whole data continuum - from sensing, to the extraction of relevant data, to its transcription, retention, analysis and, finally, to its destruction? These are essential questions.

This report begins with a review of transport safety challenges and helps position data-driven innovative solutions in the context of a wide range of interventions. The next section explores innovative solutions to address deficiencies in both the reporting and in-depth investigation of crashes. The report then examines data-led or data-informed proactive infrastructure management strategies. The following section reviews innovative solutions focusing on people and their behaviours. The final section of the report looks at issues related to retaining personal privacy, protecting commercially-sensitive information in the deployment of data-led safety strategies, and addressing organisational barriers and technical interoperability challenges. 


\section{Safe System and Vision Zero: The role of data}

Road crashes continue to kill over 1.3 million people each year (WHO, 2018), whereas not a single death occurred in a commercial passenger jet in 2017. In response to this public health challenge, the Vision Zero strategy seeks to eliminate traffic fatalities and serious injuries. It requires "a shift in how communities approach decisions, actions, and attitudes around safe mobility" (Vision Zero Network, 2018a).

Vision Zero forms part of the Safe System approach pioneered in Sweden and the Netherlands. Sweden's Vision Zero strategy was adopted by Parliament in 1997. In the Netherlands, a similar policy, entitled Sustainable Safety, was developed in the 1990s by the Dutch Institute for Road Safety Research (SWOV) in an effort to promote "inherently safe road traffic" (ITF, 2016a). The Safe System approached was endorsed by the European Commission in 2018.

According to the Safe System approach, death and serious injury in road collisions are not an inevitable price to pay for mobility. The Safe System approach accepts that people make mistakes and acknowledges that collisions will continue to occur, but it aims to ensure that such mistakes do not result in fatalities or serious injuries (Table 1). Better vehicle construction, improved road infrastructure and lower speeds can all contribute to reducing the impact of crashes, and better data can help us to understand and avoid future crashes. The responsibility for the Safe System is shared in a coordinated manner across public and private sectors. Its application is closely monitored to assess results and, if necessary, review measures, taking into account experience, new data and new technologies.

Table 1. Comparing the traditional road safety approach with a Safe System

\begin{tabular}{l|ll}
\hline Question & Traditional road safety approach & Safe System approach \\
\hline What is the problem? & All traffic crashes & $\begin{array}{l}\text { Crashes resulting in fatal and serious } \\
\text { injuries }\end{array}$ \\
\hline What is the goal? & $\begin{array}{l}\text { Reducing fatal and serious injury } \\
\text { numbers }\end{array}$ & Eliminating fatal and serious injuries \\
\hline Which planning approaches? & $\begin{array}{l}\text { Reactive to incidents } \\
\text { Incremental approach to reduce the } \\
\text { problem }\end{array}$ & $\begin{array}{l}\text { Proactively target and treat risk } \\
\text { Systematic approach to build a safe } \\
\text { road system }\end{array}$ \\
\hline What causes the problem? & Non-compliant road users & $\begin{array}{l}\text { People inevitably make mistakes } \\
\text { People are fragile }\end{array}$ \\
\hline Who is ultimately responsible? & Individual road users & $\begin{array}{l}\text { Responsibility is shared by individuals } \\
\text { and system designers }\end{array}$ \\
\hline How does the system work? & Isolated interventions & $\begin{array}{l}\text { Different elements are combined so } \\
\text { that if one fails, others provide } \\
\text { protection }\end{array}$ \\
\hline
\end{tabular}

Source: adapted from ITF (2016a).

\section{Road safety is a global issue}

The World Health Organisation (WHO, 2018) found the risk of a road traffic death remains three times higher in low-income countries than in high-income countries. Although only half of the world's motor vehicle fleet is located in low- and middle-income countries, $90 \%$ of road traffic injuries and deaths occur there (WB, 2017). For this reason, where high-income countries develop data-driven transport safety solutions, their transferability to low- and middle-income countries should be a priority. 
With their rapid penetration across the globe, their mass production and their range of capabilities, smartphones may become the world's number one transport safety equipment, helping low- and middleincome countries leap-frog richer nations. But cellular data remains an expensive and somewhat scarce commodity in many parts of the world. Many connections occur over fixed Wi-Fi networks as a result. This erodes benefits that depend on ubiquitous connectivity and real-time applications. Where possible, smartphone-related road safety innovation should be designed to deliver benefits in degraded conditions, with only occasional connections to a Wi-Fi network. Some cities choose to roll out blanket Wi-Fi coverage along their streets and public spaces. In a sense, such a policy can also facilitate the use of real-time safety-relevant apps and enable their adoption by people who can't afford a mobile data plan.

\section{Enforcement is essential}

Enforcement of traffic rules is necessary to promote safer behaviours. Not sufficient in itself, certainly better when associated with awareness campaigns, enforcement is nonetheless required.

Technology facilitates enforcement of road traffic rules with tools like automatic number plate recognition (Carnis, 2017). On the other hand, technology has provided road users with ways to evade enforcement. For instance, drivers can report the position of speed control equipment, whether fixed or mobile, effectively creating a live map that allows other drivers to reduce their speed at control sites. One should bear in mind such challenges when designing enforcement strategies which are both acceptable and effective. A better use of data, especially on speed and crash risk, seems important in this context.

Managing speeds

A range of solutions exist to bring speeds down to a level which prevents fatal and serious injuries. They include engineering, enforcement, education and nudges. The deployment of such tools would benefit from the identification of speeding hotspots in time and space and from an immediate evaluation of the results on speeds. Leveraging on new data sources, speed analytics can develop and guide the elaboration of speed and speeding management solutions.

Inappropriate speed is responsible for $20 \%$ to $30 \%$ of all fatal road crashes according to the ITF International Road Traffic Safety Analysis and Data Group (IRTAD), whose analysis confirms the very strong relationship between speed and crash risk. When average speed increases by $1 \%$, the number of fatal crashes goes up 4\%. (ITF, 2018a)

Speed is, therefore, one of the key action areas in a Safe System approach, as represented on Figure 1. Speed limits should be set and enforced to achieve safe conditions for the specific roadway context, and to protect those road users most at risk in the event of a crash. To better manage speeds, one needs to "measure and analyse the scope of problem related to inappropriate speeds, share this data to raise awareness, and develop measurable improvement strategies with timelines for action" (Vision Zero Network, 2018a). The Proactive network management section of this report makes the case for developing robust methods in the collection of spot speed distribution data. Such efforts are extremely rare at present.

Intelligent Speed Assistance (ISA) solutions are being developed to help drivers adhere to posted speed limits. Such solutions would typically involve both sign-recognition video camera and a GPS-linked speed limit database (ETSC). Implemented on a voluntary basis, the system would impose a resistance in the gas pedal when the driver attempts to exceed the speed limit. The system can be overridden by pushing harder on the gas pedal. ISA is seen as one of the most effective driver support systems to reduce the number of road deaths (Vaa, Assum and Elvik, 2014). Its feasibility and positive benefit-to-cost ratio were confirmed by Hynd et al. (2016). In 2018, the European Commission proposed that ISA is fitted on all vehicles sold in the European Union. With the uptake of ISA, mechanisms for road rule compliance begin a shift from an ex- 
post criminal enforcement approach to an ex-ante product safety approach. Such a shift was anticipated by $\operatorname{ITF}(2018 d)$ in the context of autonomous vehicle regulation.

Figure 1. The Safe System approach to road safety

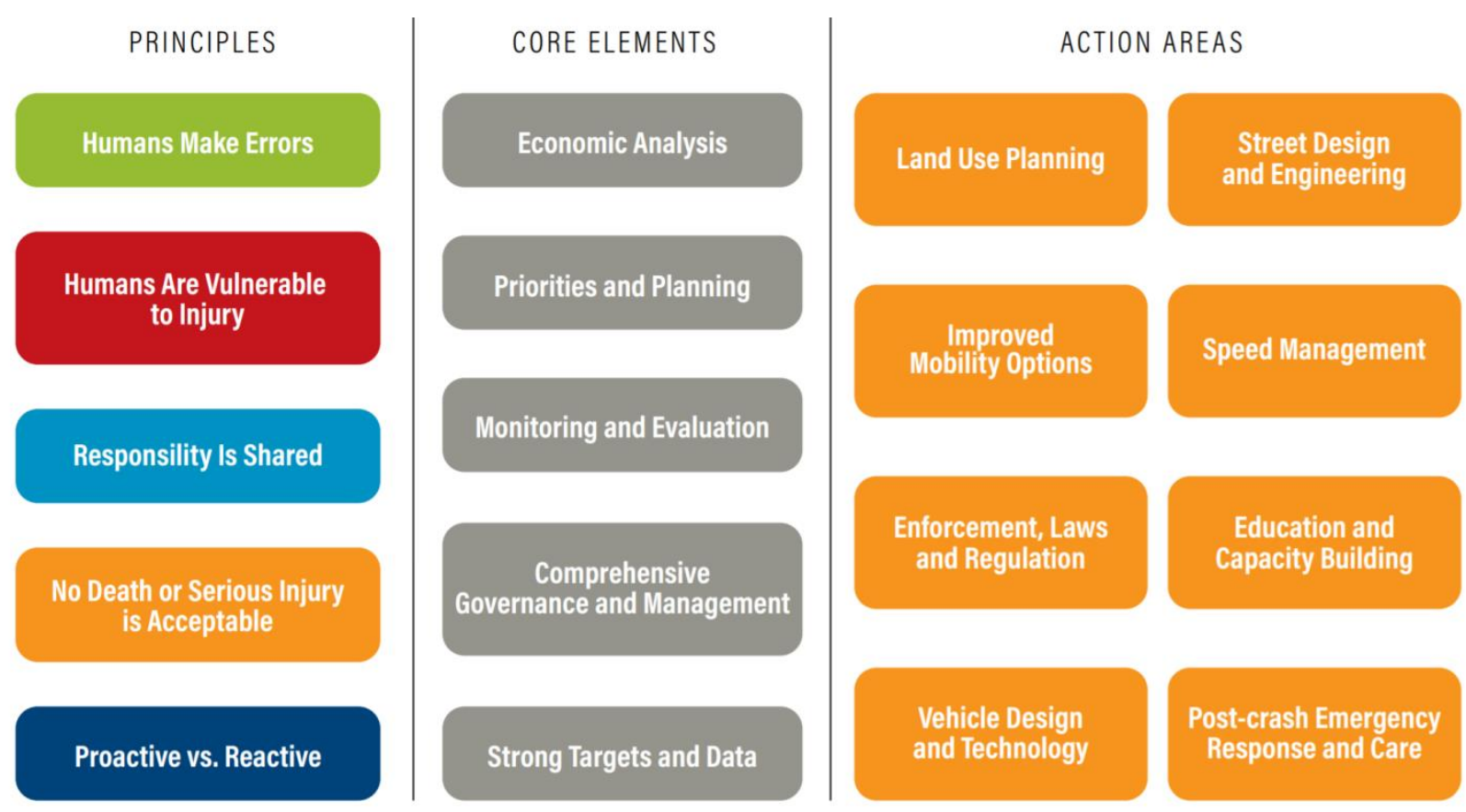

Source: Welle et al. (2018).

Following the exact same principle as ISA, geofenced speed regulations are now imposed on e-scooters in some sensitive pedestrian areas in the USA. In Santa Monica, California, e-scooter operator Lime automatically reduces scooter speed when a rider enters a geofenced area designated by the City, and returning it to normal speed as soon as the rider exits the zone (Sun, 2018). Also in California, the City of San Diego is proposing that e-scooter operators apply lower speed caps in sensitive pedestrian areas defined by a geofence (Grass, 2018).

\section{Broadening the benefits of automation}

Train automation is seen as a solution to increase network capacity, and offers transport safety benefits at the same time. Many driverless metros already exist and in 2018 the European Train Control System (ETCS) successfully illustrated the capacity for Automatic Train Operations on the mainline railway during passenger service (GRR, 2018).

Automation of road passenger and freight transport is likely over the medium term. Road safety gains could be substantial if the deployment of the technology prevents human error and follows the Safe System principles (ITF, 2018c). Yet, even in the most optimistic deployment scenarios, it will be decades before autonomous vehicles have a substantial impact on road fatalities. This is due in part to the road safety crisis being worse in countries where autonomous vehicles will likely come last.

This report therefore raises the question of what can autonomous vehicles do, even where they represent say $1 \%$ of the fleet, to deliver safety benefits. The answer could be found in the utilisation of sensors fitted on such vehicles towards a collective intelligence effort. 


\section{Protecting the most vulnerable road users}

Pedestrians and cyclists are particularly fragile in the event of a crash. In the United Kingdom, one of the few countries where data is available, their risk of fatality per unit distance travelled is more than ten times higher than if they travelled in a passenger car (DfT, 2018). This over-representation becomes more acute when considering what little threat pedestrians and cyclists present to other road users. They have benefited very little from recent car safety improvements (airbags in cars, for example, offer them no protection) and suffer the consequences of uptake of sport-utility vehicles (SUVs) in some markets.

Data-driven solutions for the protection of vulnerable road users are needed not only to protect them, but also to promote walking and cycling. Indeed, the real and perceived risks of traffic injury deter people from cycling or from letting their children walk to school, despite efforts from public authorities to encourage walking and cycling as healthier, cleaner and more efficient mobility options.

Some have advocated the uptake of technologies that "wire-up" cyclists and pedestrians so that they may be connected to the "smart" vehicular network in order to improve their virtual and real visibility to cars and trucks. This raises equity concerns given that the most vulnerable and least dangerous road users are asked to be responsible for their own safety while the most dangerous and least vulnerable road users are under no such obligation. In terms of fairness and proportionality of responsibility, it seems that cyclists and pedestrians should be "detected, not connected".

Data-driven solutions for improving the safety of vulnerable road users should not require them to carry specific equipment. Camera assisted vision, detection and warning systems seem more promising avenues for rapid and innovative safety-improving pathways. Such systems can be mounted on vehicles or on the roadside. Their value should always be benchmarked against direct vision, achieved through better vehicle design, for instance.

\section{Transit as a solution}

Innovative techniques for the collection and analysis of big data already play a key role in transport planning, as discussed by ITF (2015). As such, when data analytics support the adoption of public transport and reduce the risk of road traffic crashes, they contribute significantly to reaching road safety targets.

In urban planning, transit-oriented development (TOD) is an approach which seeks to maximise the amount of residential, business and leisure space within walking distance of public transport. This approach can include building density regulations, parking policies, urban design, and first- and last-mile facilities that support ridership and reduce auto dependency.

Where TOD principles create a transit-oriented community, residents are five-times less likely to be injured or killed in road crashes than in automobile-dependent communities. A reason for this is the relatively low risk of injury associated with public transport. In the United States, public transit commuters' per mile crash rates are ten-times less than those of automobile users (Litman, 2016). Data collected across the ITF Safer City Streets network indicates that bus travel is safer than private vehicles, not only for passengers, but also in terms of wider road safety impacts on third parties involved in collisions (Santacreu, 2018a).

Transit-oriented communities are also safer because they provide alternatives to private vehicle use for individuals who consume alcohol, take medication or suffer from poor eyesight or any other condition that may impede driving capacity. Alternative mobility options provide teenagers with substitutions for cars and mopeds, and allow older people to stop driving when they wish. Transit-oriented developments include facilities for walking and cycling, reduce traffic and further enhance the safety performance.

Overall, diminished dependence on private motor vehicles reduces traffic volumes and contributes to transport safety (Ahangari, Atkinson-Palombo and Garrick, 2017; Litman, 2019). Traffic volume reduction 
has traditionally been overlooked as a road safety policy. Travel demand management, multi-modal planning and more efficient pricing should be accepted as Vision Zero policies. Car-dependent communities often result from poor land-use planning. Planners should seek to create denser neighbourhoods, and create permeability by means of a direct pedestrian and cycle network.

Public transit is also a relevant solution to improve road safety in low- and middle-income countries. For instance, the introduction of the Macrobus, a mass transit system, in Guadalajara, Mexico, has led to a $46 \%$ decrease in road traffic injuries (WB, 2017). 


\section{Gathering intelligence on crashes and injuries}

Traffic crash information is fundamental in diagnosing safety problems, assessing crash costs, monitoring progress towards targets and guiding decision-making. Inaccurate crash records may bias the results of traffic safety analyses, leading to misguided crash-prevention strategies and resulting in wasted efforts. Including non-severe injury data, which are notably missing or inaccurate in many crash statistics, helps in understanding how safety, or the lack of it, is experienced and perceived by people and how this impacts their travel decisions.

Most countries rely on police data to count road traffic crashes, which results in systematic under-reporting of injury data, especially slight injuries (ITF/OECD, 2011). The level of under-reporting varies according to the profile of people injured, e.g. by mode or demographic group, which is skewing analytical results. Lowincome communities, where people often suffer from a lack of trust in the police force and insufficient health insurance, are particularly likely to under-report crashes. Some crashes may simply be out of scope for police data collection. For instance, national crash databases are typically designed to exclude pedestrian falls.

In a report focusing on low- and middle-income countries, the World Bank (WB, 2017) identifies the lack of reliable data as a challenge to understand the aggregate impact of road traffic injuries on economic growth. It recommends improving surveillance systems such as data collection instruments to better assess the causes, extents, and risks associated with road traffic injuries.

\section{Merging data from police, hospital and insurance sources}

Several sources of data exist to assess the number and characteristics of crashes as well as their health outcomes: police records, coroner's reports, and data from the insurance and health care sectors.

In low-income countries, $80 \%$ of road fatalities are not included in official statistics (Krambeck, Job and Sultan, 2018). As a result, there is no trusted baseline against which authorities may monitor progress. In response to the situation, the World Bank developed a tool for crash data management which:

- links multiple agencies involved in recording road crash data-local government units, the police, and the health system

- $\quad$ standardises terms and definitions for reporting, and

- provides analytical tools to support evidence-based investments and policies and to monitor the impact of interventions (Krambeck, Job and Sultan, 2018).

The tool is called DRIVER (Data for Road Incident Visualization Evaluation and Reporting). It was developed and deployed in the Philippines, and is now available on an open-source platform for other governments to adopt. To further facilitate adoption, it feeds on free OpenStreetMap data. Tools such OpenStreetMap, a collaborative project to create a free editable map of the world, are an essential building block towards the implementation of road safety polices.

\section{Hospital data}

In many countries, hospital data are mainly used for medical purposes and seldomly for official statistics on road safety (ITF/OECD, 2011). This is a missed opportunity. Yet things are slowly changing as more cities and countries are now using hospital data to better assess the number of people injured in road crashes. In the United States, for example, Portland officials collected data from emergency rooms across the county during a four-month e-scooter trial to assess the number of associated injuries (PBOT, 2019). In the city of Barcelona and in the French Rhone area, permanent data collection on crash circumstances is conducted in 
hospitals. This dynamic benefits from the support of the European Commission, which seeks to accurately monitor the number of serious injuries across member states. The Commission promotes a harmonised injury severity scale across countries, the Abbreviated Injury Scale, which requires a medical assessment.

Several challenges remain associated with hospital data collection. The main pitfall is when the source of injury is not rightly coded. Another challenge is the need for a consistent injury severity scale, essential for both monitoring and benchmarking purposes.

\section{Ambulance data}

Not all crashes require police intervention. However, many crashes not attended by the police do attract emergency medical services and open opportunities for gathering data. In such situations, ambulance services could encourage the victim to post a self-report to the police at a later stage. Emergency medical services could also share data electronically and systematically, to the police and highway authorities, on injuries resulting from falls and road traffic crashes.

Initiatives involving electronic recording of ambulance patients already exist. They have been made possible by beforehand standardisation of ambulance data. In the United States, the National Emergency Medical Service Information System (NEMSIS) defined a standard for aggregated ambulance data, data that it stores and shares. In 2016, the NEMSIS database included over 30 million data points submitted by ten thousand ambulance agencies active in 49 US states and territories (NEMSIS, n.d.). In the United Kingdom, the National Health Service (NHS) sponsored a similar project. Completed in March 2014, it resulted in a new national standard for the structure and content of the clinical records of ambulance patients (College of Paramedics, 2014). Based on this standard, the implementation of electronic patient clinical records (ePCR) was discussed (Porter et al., 2018).

While the aim of these efforts towards digitalisation and standardisation is to improve the health system's efficiency through better cooperation between health providers, the resulting high-quality data could complement police and hospital sources to provide a more comprehensive understanding of road safety.

\section{Smartphones}

Smartphone apps are often used for journey planning and navigation. Citymapper, Google Maps and Moovit provide a number of options for a single destination and include multiple modes of transport. Strava, GeoVelo and Bike Citizens are dedicated to finding cycling routes. Specific apps could also be developed allowing users to report crashes they witness or are involved in, but it seems unlikely that large numbers of the public would install an app which is used only on extremely rare occasions.

Instead, crash reporting solutions could be integrated in existing apps where citizens can report broader issues (such as FixMyStreet, described further down in this report). Bridging municipal boundaries, such apps should best be developed at the national level to gain and retain a large amount of contributors. Smartphone apps could ask their users to rate their journey in terms of safety. Citymapper, for example, already seeks to assess the security of late journeys.

Partnership between app developers and governments could generate valuable insights. If and when wearable devices become widely adopted, such apps could seek to detect close call incidents through heart beat monitoring and crashes from accelerometer readings. They would later prompt the user to confirm if an incident happened and seek a self-report in this case. Less serious than crashes, yet more frequent, close call incidents significantly affect people's quality of life and mobility choices. Collecting data on such incidents should be on the radar of policy makers (Aldred and Crosweller, 2015). 


\section{Data linkages}

In most countries, two separate sources of information are often used by road safety practitioners when assessing the number and characteristics of crashes and their health outcomes. One is the police crash database, particularly appropriate to record crash time, location and vehicles involved. However, a police database fails to capture the total number of serious injuries due to under-reporting. Another source is health records, which - ideally - attribute a cause to each death and log hospital treatments attributed to each disease code (and therefore to road crashes). Health records could reflect close to $100 \%$ of serious injuries but rarely contain any reliable information on crash circumstances.

Police data describes crash location and vehicles involved, while hospital data captures accurate injury details. The strength of one data source is the weakness of the other. For this reason, linking police and hospital datasets proves valuable (Pérez et al., 2016; Pérez et al., 2018). Several linkage methods are described by the IRTAD Group (ITF/OECD, 2011). Several cities, members of the ITF Safer City Streets network, have reported running linkages on a regular or ad-hoc basis. The operation is probabilistic by nature due to inaccuracies in both police and hospital datasets (Box 1).

\section{Box 1. New York City matches police and hospital records}

The New York City Departments of Transportation and Health worked together to the linkage of traffic crash and hospitalisation records. A probabilistic matching exercise was conducted using LinkSolv based on 2009-2013 data. A total of 145000 liked pairs were found, that is $52 \%$ of total hospital traffic injury records, or $41 \%$ of those crash records expected to be hospitalised.

The analysis was used to validate the quality of injury severity assessments conducted by the police on-site, but also to identify potential improvements to its accuracy. Local police data records injuries on the KABCO scale ( $K=$ Killed, $A=$ Serious injury, $B=$ Moderate injury, $C=$ Complaint of injury, $O=$ No injury). On the other hand, hospitals record injuries with ICD-9-CM E-Codes (E810-E819 for motor vehicle related crashes) and injury severity score (ISS ranging 1-41).

Nine variables were used in a probabilistic matching protocol: age, sex, crash county (hospital county), crash date (admission date), crash hour (admission hour), crash role, collision type, injury body location and severity.

Source: NYC DOT (2017), Conderino et al. (2017).

Insurance data could also be of value, where insurance companies agree on sharing this information (Box 3). Damage-only crashes are particularly likely to be under-reported in police records, if not simply out of scope. Yet, damage-only crashes could prove relatively frequent and particularly well-suited to data mining. Such aggregate analysis could reveal insightful patterns such as hotspots and crash risk factors.

According to the European Transport Safety Council:

States that use a single source of data, usually police records, should consider cross-checking with other sources such as hospital data and coroners' records. Research in countries that do use more than one source, reveals that single sources tend to be incomplete. Even deaths go unrecorded by police in countries that checked this with other sources, for example, in cases where police were not called to the scene of a collision. Death and injury in collisions that do not involve motor vehicles can also be missed in national statistical data in some countries due, for example, to the fact that hospital and police records are not cross-checked routinely. (Adminaite et al., 2018) 


\section{Self-reporting falls and crashes}

Self-reporting is a common way to complement official records and address the under-reporting of crashes, some of which may never be reported to the police, hospitals or insurance companies (Kamaluddin et al., 2018). Advantages of self-reporting are presented by Polders and Brijs (2018):

- First-hand information is collected from the involved road user

- Under-reporting is mitigated in comparison with official statistics

- Information regarding near-crashes can be collected

- Data collection can be tailored for a specific research question or road user group

- Aspects that are not covered in official statistics can be included

- Trends in accident statistics can be revealed at an earlier stage

- Evaluation of traffic safety measures can be conducted earlier.

Self-reported injuries can be five-times more numerous than those recorded in police collision data. Selfreport surveys would reveal the true extent of injuries and reveal how the scale of under-reporting varies across user groups (Box 2).

\section{Box 2. Self-reported injury data from the British National Travel Survey}

Until recently, police and hospitals were the sole source of the United Kingdom's road injury data, with the exception of coroners' reports. However, since 2007 the National Travel Survey has asked about "road accidents" on the public highway, including single-bicycle incidents and pedestrian falls. The data presents a different angle on injuries based on the perspective of those involved rather than police or hospital staff. There will still be some under-reporting, related to ability to recall or admit to involvement, which may differ by incident type.

The findings demonstrate that self-report injuries are around five-times more numerous than those recorded in police collision data. For cyclists, the level of apparent under-reporting is higher, with almost seven-times as many incidents reported in the NTS data as are recorded in police files. However, these incidents contained a relatively high proportion of injuries where medical attention was not sought.

Source: Aldred (2018).

Surveys could help estimate and monitor the actual number of people injured in traffic (Santacreu, 2018a). Self-reported data could complement hospital and police data, despite known survey limitations such as non-response, social desirability bias or recall bias (Kamaluddin et al., 2018). Social desirability bias is the tendency of survey respondents to over-report "good behaviour" or under-report undesirable behaviour. Recall bias is a systematic error caused by inaccurate or incomplete responses by study participants regarding events or experiences from the past.

Smartphones appear to be a promising channel for collecting self-reported data. Smartphones and wearable smart devices can also offer the opportunity to record data on falls and crashes. There are ongoing efforts to develop these types of sensor-based apps (Madsen et al., 2017).

Developing countries should increase their efforts when it comes to using self-reporting to better assess the actual traffic safety situation and elaborate appropriate safety measures (Kamaluddin et al., 2018).

Developped countries should also acknowledge the weaknesses of their reporting systems. All countries should therefore consider undertaking self-report surveys such as that included in the National Travel Survey in England. 


\section{Box 3. Self-reporting of road crashes with smartphone apps in the Netherlands}

Police records in the Netherlands desperately lack in crash data. Recognising that the absence of such information weakens data-driven road safety diagnostics, monitoring and evaluation, three organisations have joined together in an effort towards the complete registration of all traffic crashes: the national police, the Association of Insurers and software firm VIA. Under the name Smart Traffic Accident Reporting (STAR) the group collaborates to combine police information and insurance data and fill the data gaps.

Eleven national stakeholders signed an agreement to facilitate crash reporting by road users. The STAR initiative launched a smartphone app called MobileDamageReporting in March 2016. It enables individuals to report road crashes, and allows for support and (partial) verification by police or insurance companies. The app uses GPS to determine location and allows for the reporting of crashes involving no motor vehicle, such as bicycle-only crashes. The quality and comprehensiveness of the resulting data set is yet to be assessed, but hopes are that serious bicycle crashes that do not involve motor vehicles will be reported with this app.

In the event of a crash, those involved are encouraged to report the collision with the simple, user-friendly app on an online platform. When crashes involve motor vehicles, the app uses the vehicle license plate to collect vehicle and insurance data automatically, as entering the postcode and house number suffices to ascertain the address details of the people involved.

Insurers, who have a huge interest in limiting injury and damage, welcome MobileDamageReporting as a customer-friendly, efficient replacement for the European damage claim form. Road managers may also benefit from the app, as they will gain improved insight into infrastructure safety and relevant information on crash causes. This makes preventative measures much more efficient to apply and substantiate.

Source: ITF (2016b).

Pedestrian and two-wheeler falls

Pedestrian falls in the public space are often excluded from the scope of police data collection. Similarly, falls from bicycles and powered two-wheelers are seldom reported to the police. However, they represent a significant social cost in terms of health care and absence from work. They also present an opportunity for people managing the transport system to learn from minor crashes proactively, instead of reacting to fatal or serious injuries after they occur.

Falls could be detected by roadside computer vision surveillance systems integrated into infrastructure. Video camera feeds linked to computer vision systems with scene extraction and scene replay capabilities can capture relevant events, such as crashes, falls and near misses with very accurate geographical information. As the number of cameras filming public street- and curb-space increases, the level of algorithmic skill improves, and the price and size of sensing-processing computer chips falls, it may be worth requiring that such systems be mandatory safety equipment for surveillance cameras. Just as vehicles are mandated to have certain types of safety equipment, public authorities could include stipulations in the licensing of street and sidewalk surveillance cameras, requiring on-board edge-computing sensing and incident detection chips and algorithms. These could automatically extract relevant scenes for statistical and forensic purposes. After a buffer period (necessary for forensic post-crash analysis), these systems would only produce aggregate statistics rather than individual video files.

\section{Crash reporting in the bike-sharing and scooter-sharing sectors}

Governments could seek to impose the provision of relevant crash data on bike sharing or e-scooter sharing operators, either through procurement or partnership. Such data could include hard braking events, falls, and user feedback following falls. Portland successfully instituted data-sharing requirements as a tool to 
assess the impact of e-scooters (Box 4). Such requirements should, however, not become a barrier to innovation, hindering the potential road safety and public health benefits that may be derived by replacing a number of car journeys with shared micro-mobility solutions.

\section{Box 4. 2018 Portland e-scooter routes travelled}

Against a national backdrop of unforeseen and unpermitted e-scooter launches, the Portland Bureau of Transportation (PBOT) took a proactive approach to managing shared e-scooter services. They established a permitting framework to run a 120-day e-scooter pilot which required operators to agree to a set of comprehensive data-sharing requirements. Data specifications were modelled after the Los Angeles Mobility Data specification to include availability, trips, collisions and complaints.

Data revealed where and when e-scooters were used. Real-time data facilitated the monitoring of compliance with deployment requirements. Data collection was supplemented by a rider survey, citywide poll, focus groups, an online complaint form, and community and stakeholder input.

Informed by company-provided route data, the map on Figure 2 shows routes travelled by e-scooter riders most often. Darker blue dots signify more trips taken on that street segment.

Figure 2. Portland e-scooter traffic density

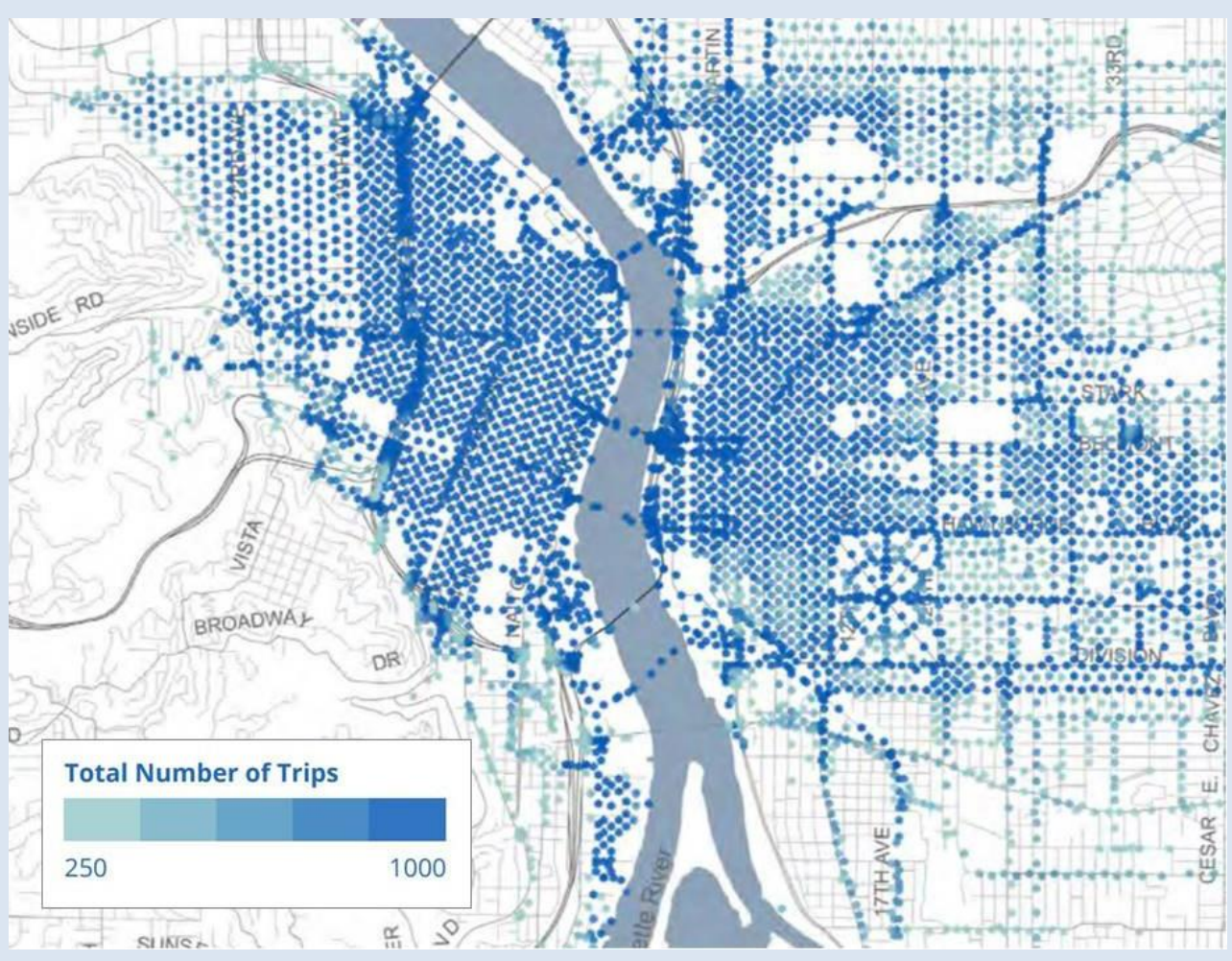

Source: PBOT (2019).

By requiring bike and e-scooter sharing operators to report falls and crashes, governments would take advantage of sensors already built into the devices. This approach consists in capturing data which would otherwise be wasted. Following the same principle, governments should also require the automotive industry to report engagements of ABS / ESP / AEB systems, to complement the automatic crash notification system (eCall in Europe). 
Shared micro-mobility devices are not used evenly across cities. Their crash data, once mapped, will most likely reveal where most people are riding rather than particularly risky locations. This is why particular attention should be placed on collecting appropriate exposure data, as was done in Portland.

In addition to the collection of falls and exposure data, one could see value in other data from shared micro-mobility operators. People going the wrong way down one-way streets could be mapped to reveal areas where two-way cycling facilities would be popular. Riders turning across multiple traffic lanes and across oncoming traffic could also be mapped, so that planners could work on street design solutions to protect them. More generally, automatic video scene analysis could help reveal pedestrian and cyclist desire lines and help inform safe infrastructure layout that supports these. People crossing a busy road unprotected could be identified by video analytics, thus making the case for additional crossing points or speed management measures.

Bike and e-scooter sharing operators have not yet established a consistent reporting methodology for capturing falls and crashes. There would be value in developing a harmonised industry-wide reporting framework, in partnership with local government and operators. GPS and motion sensors create an obvious opportunity to automatically detect and gather intelligence on falls and crashes: where and when they occur, the speed of the rider, etc. Since all users are identified customers, operators could send a push notification through the app asking the rider whether such an incident has taken place. When a rider reports a crash to an operator, some of the key attributes that should be included in what should be a universal crash data collection tool are, according to Fishman and Schepers (2018): name; date of birth; gender; crash date, time and location (using online mapping tool to provide geographic coordinates); crash type; whether or not police were called to the site; injury severity; injury details; customer consent for safety researchers to contact the victim; and contact details.

\section{In-depth crash investigation using Event Data Recorders}

A major difference between road transport and other modes is the general lack of a formal, independent crash investigation body. Police may investigate individual road crashes but lack the resources and techniques of a dedicated crash investigation body (ITF, 2018b). As a result, only a small number of countries conduct in-depth road crash investigations in a systematic way.

\section{Event data recorders}

Road crash investigation often seeks to derive vehicle speeds from tyre skid marks or impact severity. Tyre marks have been eliminated by the ABS technology found on recent vehicles, leaving investigators with one less piece of evidence. However, some road vehicles are equipped with an Event Data Recorder (EDR), which is "a system for recording vehicle data during unintended events with harmful outcomes (i.e. damage or injury), with no continuous monitoring of driver behaviour or performance" (Hynd and McCarthy, 2014).

EDRs typically take the form of a device installed on a motor vehicle to record data immediately before, during and immediately after a crash for a brief period of time which is less than a minute in total. The recorded information may include pre-crash vehicle dynamics and system status, driver inputs, restraint usage and deployment status, and post-crash data, such as the activation of an automatic collision notification system (NHTSA, 2005). EDRs usually do not save and store audio and video information, although Italy has installed video-enabled EDRs in thousands of ts public transportation vehicles (EP, 2014). 


\section{Box 5. NHTSA Event Data Recorder standard}

In the United States, EDRs are imposed and are found on all new cars. The United States National Highway Traffic Safety Administration (NHTSA) requires that EDRs record, in a readily usable manner, data valuable for effective crash investigations and for analysis of safety equipment performance, through regulation Part 563 . These data will help provide a better understanding of the circumstances in which crashes and injuries occur and will lead to safer vehicle designs. The data must include:

- Forward and lateral crash force

- Crash event duration

- Indicated vehicle speed

- Accelerator position

- Engine revolutions per minute (rpm)

- Brake application and antilock brake activation

- Steering wheel angle

- Stability control engagement

- Vehicle roll angle, in case of a rollover
- Number of times the vehicle has been started

- Driver and front-passenger safety belt engagement, and pretensioner or force limiter engagement

- Air bag deployment, speed, and faults for all air bags

- Front seat positions

- Occupant size

- Number of crashes (one or more impacts during the final crash event)

To promote a consistent interpretation of Part 563 regulation across the industry, the Society of Automotive Engineers (SAE) Event Data Recorder Committee develops technical reports that produce common data output definitions and formats. Such standards seek to facilitate retrieval and interpretation of data from EDRs.

Source: SAE (2018).

Crash data retrieval from EDR units involves a standard communication port on the vehicle called on-board diagnostic or OBD. Crash investigation experts in some countries have, however, expressed great difficulties in accessing EDR data and making an independent interpretation of its contents. This shows that imposing a device is not enough to guarantee access, and common data formats are of critical importance. Several experts raised this as an issue in Europe.

Crash investigators use EDR data to reach a better understanding of the sequence of events leading to a crash. The goal is to enable the police, crash investigators, manufacturers and researchers to obtain more objective knowledge about the causes or factors contributing to a collision. Through crash investigation, EDR data can help the automotive industry refine the design of vehicle safety systems, ultimately reducing the number and severity of crashes. This is particularly important at a time when new driver assistance systems are multiplying. The risk of failure or misuse in such new systems shall certainly be assessed and monitored through in-depth crash investigation.

EDR data could also be used as evidence in court to help determine liability. In some countries like the United States, courts can already request EDR data for this reason (EC, 2015a). EDR data has also been used as evidence in court cases involving severe injury or damage in the United Kingdom and in Australia (Technavio, 2017). However, since regulation regarding EDR data privacy and ownership is not standardised, court use varies from country to country and from state to state. The use of EDR data in court may contribute to improving driver accountability and, therefore, behaviour. 
In short, EDRs provides "more objective, reliable and cost-effective evidence of crash severity, pre-collision driver behaviour, vehicle system performance etc. than is currently available via e.g. crash reconstruction, witness statements or CCTV records" (Hynd and McCarthy, 2014).

Looking ahead

Experts reported that the People's Republic of China and Japan are both working to develop pieces of legislation on EDR which could be relatively close to the US legislation, but broader. The European Commission proposes to mandate EDR on all new cars and considers its introduction as "an important step in the right direction to obtain an EU-wide in-depth accident data, which does not exist today on an enough extensive scale, but is indispensable for a comprehensive monitoring of the road safety performance of vehicles" (EC, 2018a).

For commercial vehicles, Hynd and McCarthy (2014) report that: "Analogue and digital tachographs record very little information about a collision compared with the EDRs often fitted to cars. In the United States, NHTSA started working on EDRs for heavy goods vehicles and buses at around the same time that it started work on EDRs for cars, via the NHTSA Truck and Bus EDR Working Group."

EDR recording is typically triggered when airbags are deployed. As a result, collisions involving vulnerable road users are most likely not recorded. In the United States, the part 563 specifications are not adequate for triggering on such impacts (Hynd and McCarthy, 2014). Therefore, future regulations on technical requirements should seek to define triggering conditions which include pedestrian and cyclist collisions.

Legal issues on data ownership and privacy are serious obstacles to using EDRs to their full potential. It is therefore essential to protect user privacy by defining who has access to the data and under which circumstances. In the United States, "the Black Box Privacy Protection Act" has been proposed with the objective of giving vehicle owners more control over the information collected in a light vehicle EDR. It would make it illegal for anyone other than the vehicle owner to retrieve information without owner consent or a court order (Hynd and McCarthy, 2014).

Looking further ahead, regulators may impose specific incident self-reporting requirement to manufacturers of autonomous vehicles. The scope of such requirements could include close calls, in which case the next generation EDR would simply store sensor data until a suitable mobile data connection or Wi-Fi connection became available and data was transferred to the manufacturer's servers. Setting standards and common definitions for EDR data in close call incidents was investigated in the context of autonomous vehicle testing, but remains a challenging task (Nowakowski et al., 2015).

\section{Automatic crash notification}

Since April 2018, all new cars sold in the European Union have been equipped with the automatic crash notification system eCall. eCall communicates a vehicle's exact location to emergency services, the time of incident and the direction of travel, even if the driver is unconscious or unable to make a phone call (EC, 2015a). It can also be activated manually or vocally by witnesses or passengers.

The regulation for the automotive industry to include eCall on all new cars was based on the unacceptably high number of road deaths in the European Union (EC, 2017; EP, 2018) and the difficulty of emergency services to obtain precise crash locations, leading to a delay in emergency responses (EP, 2015). The common European emergency number 112 naturally facilitates the implementation.

eCall is a dormant one-way communication system believed to be immune to cyber-attacks. As such, privacy protection is another of the system's strengths, achieved through the minimisation of data collection: 
- location is communicated only in the case of a crash (Guilbot, Vaslin and Arregle, 2018)

- the dataset communicated following a crash is limited to: vehicle type, fuel used, time of the crash, exact location and number of passengers (EP, 2018).

It is unclear if and how eCall could be triggered in crashes involving vulnerable road users, which rarely result in airbag deployment. Pedestrians, cyclists and riders of powered two-wheelers would nonetheless benefit from the manual activation of eCall by crash witnesses.

There are technical challenges associated with the detection of crashes with vulnerable road users, but they are surmountable. Progress achieved in autonomous emergency braking (AEB) could be adapted to eCall. In other words, where a system exists to monitor the risk of pedestrian and cyclist collisions ahead, it triggers eCall in the event of a crash which AEB failed to avoid. In cases like hit and runs with no witnesses, such a system could save lives. 


\section{Proactive network management}

In a traditional approach to transport safety, infrastructure managers rely on crash data to identify the need for interventions. This is a reactive measure, where people must be seriously injured before problems are addressed. A Safe System proactively heads off injury. By conducting a thorough analysis of risk factors and incidents, a Safe System sets out to predict risk levels and intervene before serious crashes happen. Relevant data might include network geometry, infrastructure defects, risky behaviours and close call incidents. This section of the report examines the avenues for developing proactive infrastructure management using technological enablers, such as video analytics, which didn't exist or weren't scalable as recently as five years ago.

\section{Surrogate safety metrics to power crash prediction models}

Crash numbers are not an effective metric for assessing the safety of a specific site due to the relatively small number of crashes at any given location. Many years of analysis must be compiled to adequately assess the safety situation of a specific site. Another reason is that many crashes are never reported to the police. The share reported varies from site to site and between different road user groups. A third reason is that countermeasures are often introduced to sites due to a high number of reported crashes: any future drop in the number of crashes is then often attributed to the successful implementation of the countermeasure, whereas the drop would most likely have happened without intervention, through a statictical phenomenon called "regression to the mean", explained in Box 6.

\section{Box 6. Regression to the mean}

In most before-and-after studies reported in road safety literature, remedial measures have been deployed after a period of "unacceptably high" collision counts. However, a period of high collision counts at a specific location is often due to random fluctuation in relatively small collision numbers. Due to these random fluctuations, it is likely that collision counts will return to a lower baseline level in subsequent time periods, regardless of the value of the intervention. This natural statistical phenomenon is known as regression to the mean.

Figure 3. Separating regression to the mean from the true benefit of an intervention

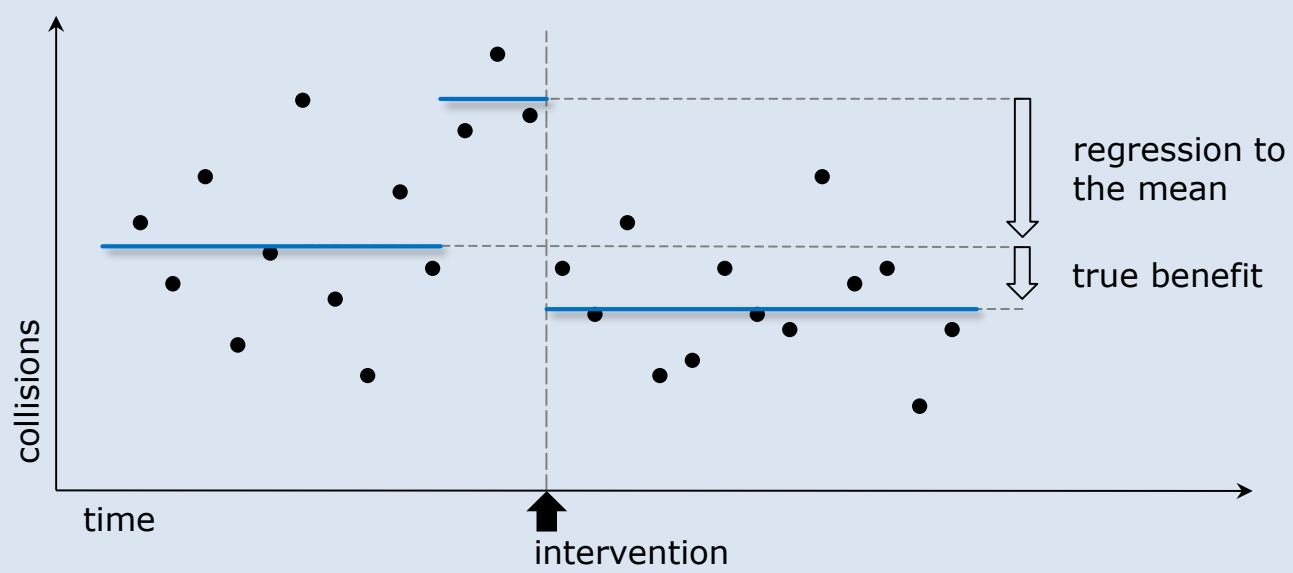

Studies assessing the effectiveness of road safety schemes are notoriously bedevilled by the problem of regression to the mean. In Figure 3, most of the change observed immediately after a road safety intervention would have been observed anyway, even without intervention, simply by regression to the mean.

Source: adapted from Fawcett et al. (2017). 
For the reasons above, a number of researchers argue that road safety analyses would strongly benefit from reliable methods that utilise observable non-crash events as a surrogate or a complement to crashes. Relevant non-crash events, also called conflicts or close-calls, often involve evasive action such as braking or swerving. The assumption is that correlations exist between crashes and conflicts. Predictive power would therefore be found in the analysis of conflicts. Hydén (1987) uses a "safety pyramid" to describe this relationship. The base of the pyramid consists of normal traffic encounters that are quite safe and frequent. The tip of the pyramid contains the most severe events, such as crashes resulting in injuries or fatalities, which are highly infrequent.

Figure 4. Hydén's safety pyramid

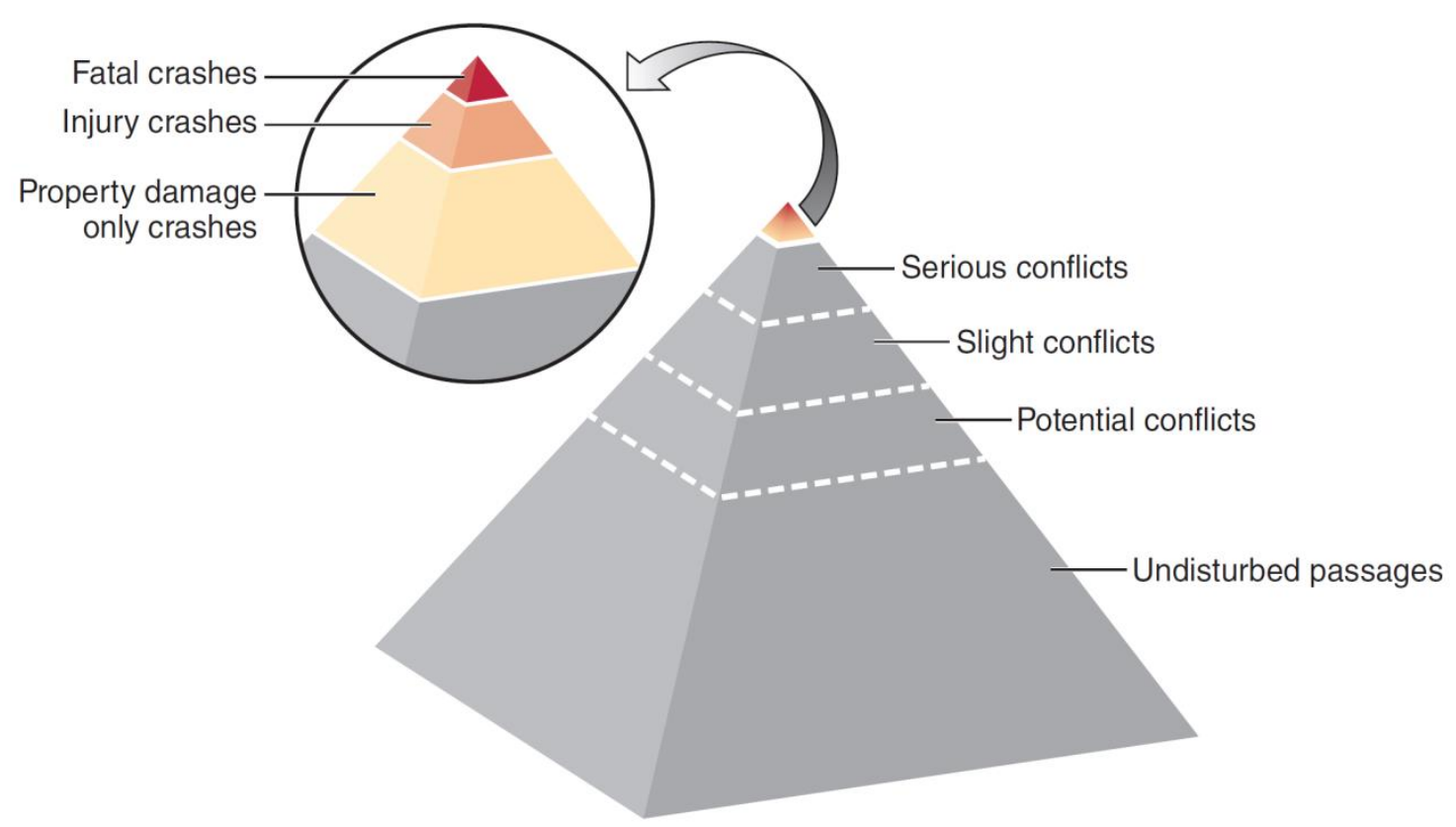

Source: Chang, Saunier and Laureshyn (2017), adapted from Hydén (1987).

How to distinguish conflicts from undisturbed passages? How to identify the most serious conflicts? Several metrics exist to quantify how close a situation is to a crash, a concept called proximity. Two primary metrics were identified by industry experts:

- $\quad$ Time to collision (TTC) is the time required for two road users to collide if they continue at their present speed and on the same path.

- $\quad$ Post-encroachment time (PET) is the lapsed time between the moment that the first road user leaves the conflict area, and the moment that the second road user reaches the same area.

The primary goal of Vision Zero is to avoid severe crashes, rather than all crashes. For this reason, traffic conflict indicators should take into account the severity of the injuries resulting from a collision had it occurred, but very few currently do (Laureshyn et al., 2017). Conflict severity indicators should include crash participants' fragility, speeds, masses and crash angles.

Surrogate road safety analysis is set to feed from vast numbers of CCTV cameras. Where several cameras share the same field of vision, techniques will evolve to track movements with greater accuracy. The stereoscopic camera illustrates the added value of data fusion across multiple sensors. To remove ambiguous image interpretation and to compute more accurate trajectories, the analysis would also benefit from LIDAR sensors coupled with camera images. 
A diversity of behaviours can be analysed with computer vision. Compliance in situations where vehicles must yield to pedestrians can be scored from video analytics (Fu, Miranda-Moreno and Saunier, 2018). Passing distances can be monitored on a specific site to assess cycling safety improvements following changes in the street layout (Götschi et al., 2018)

\section{Box 7. Predicting collision counts at potential road safety hotspots}

Road safety practitioners can now use sophisticated statistical modelling techniques to predict accident counts at potential road safety hotspots. Researchers from Newcastle University in the United Kingdom have developed such a tool, involving a Bayesian hierarchical model (Fawcett et al., 2017). The method has been implemented in a tool called Reactive Analytic Prediction Toolkit for Road Safety (RAPTOR) and is now also available within the commercial software PTV Visum Safety (Figure 5).

The method allows for more proactive decision-making on where to implement road safety schemes, like speed cameras, for example. This is different to the reactive approach currently taken by most road safety organisations, where such countermeasures are often deployed only after a threshold of fatalities or serious injuries has been exceeded over an observation period.

Not only is this method more proactive, it may also prevent unfit road safety investments based on the misinterpretation of naturally volatile crash figures.

The municipality of Lisbon, in Portugal is already working in close collaboration with Newcastle University to develop prediction models that will be used in the context of a wider "Sustainable Mobility Plan" for the city.

Figure 5. Predicted hotspot location mapped in Visum Safety

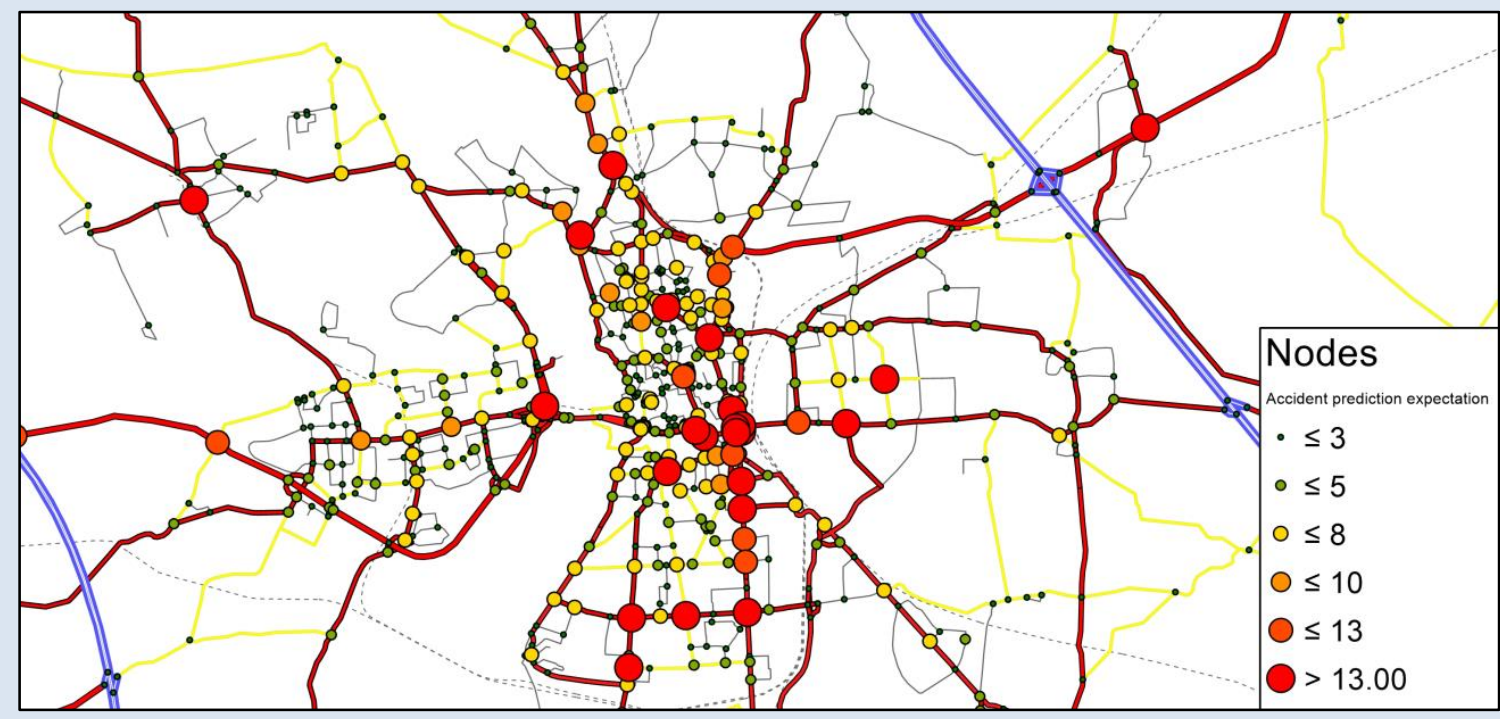

Source: Newcastle University.

A galaxy of surrogate road safety metrics could be defined, used, and potentially misused. Shared standards should be established so that research results may be comparable and transferable. The need for harmonisation and standardisation of terms, definitions, and techniques for surrogate safety has been recognised for decades (Chang and Wilson, 2018).

A precondition to scaled-up surrogate safety is the development of skillful and transparent surrogate safety algorithms. Artificial intelligence (AI), and more specifically, machine learning algorithms are uniquely suited to this task but they must be trained on representative data. The use of these raises general questions about which training data to feed into surrogate safety AI algorithms. It also raises the question 
of whether the explainability of the algorithmic output is retained where machine-learning systems re-write their code on the fly (for further discussion of governing algorithmic systems see ITF, 2019b).

Naturalistic studies collect continuous data (position, speed, acceleration, swerving, manoeuvres and video of surroundings) unobtrusively from road users' own means of transport during daily travel. This information is used to study road user behaviour. Naturalistic studies - often applied to the behaviour of a sample of road users - can produce a number of surrogate safety metrics which include speeding, braking, and specific manoeuvres (Polders and Brijs, 2018). Such studies are facilitated by the availability of compact, lightweight equipment capable of recording GPS location, video and accelerometer signal continuously, and are easily fitted to bicycles or cars.

\section{Mapping risk factors along the network}

The concept of surrogate safety isn't limited to fixed sites, such as junctions, where most camera equipment is available. Floating vehicle data can also provide information. For example, hard braking events revealed by GPS trajectory data can be used as a proxy for incidents and can help predict crash hotspots (Strauss et al., 2017; Stipancic et al., 2018).

In New York City, driver behaviour was mapped using real driving information collected through a smartphone app called Dash (Daud et al., 2018). The analysis revealed that $92 \%$ of drivers operating on 50-MPH streets late at night were driving above the posted speed limit. It also resulted in a map of hard braking events. It is unclear, however, if and how the number of events is normalised for the underlying probe traffic volume. Failure to normalise the data may produce a map which reflects predominantly the probe vehicle traffic patterns rather than hard braking intensity. This is particularly problematic where probe vehicles are concentrated in specific areas or on specific street types.

\section{Box 8. Data reveals systematic inequalities between neighbourhoods}

Across the United States, $90 \%$ of high-income neighbourhoods' streets have sidewalks, compared to only $50 \%$ of those in low-income neighbourhoods (Vision Zero Network, 2018b). Interesingtly, minority ethnic groups and low-income communities carry a disproportionate burden of traffic related injuries and fatalities (Vision Zero Network, 2018a).

In Los Angeles, the Vision Zero task force identified a High Injury Network (HIN) where $64 \%$ of the killed and severely injured occurred on what represented $6 \%$ of the road network. Their analysis went further to demonstrate that nearly half of those streets are found in communities already burdened with the poorest health and economic conditions (Vision Zero Network, 2018b).

In the United Kingdom, using a method based on self-report, Aldred (2018) shows that living in a household earning below GBP 25000 is associated with more than double the odds of injury. The self-reporting method is crucial in reaching this finding because low-income groups tend to have less access to hospitals and be more wary of authorities, making them less likely to report accidents they are involved in.

A wide range of factors may explain the disproportionate burden of traffic-related injuries in low-income communities. Age, mode choice and behaviour may be among these factors, but more systematic factors should not be overlooked. In the United States, for instance, African-American communities have been historically left out of transportation planning, have suffered continuous under-investment and are thereby lacking basic road safety amenities. Moreover, in Europe and more recently in the United States, gentrification has contributed to push lowincome groups out of city centres where streets are often safer due to shorter blocks and narrower streets (Austin City Council, 2016). 
In London, Ford has collected data from 160 vans to correlate vehicle dynamics with known crash hotspots, and predict future crash locations (Ford Smart Mobility, 2018). Floating vehicle data included speed and decelerations. The outcome is available in a report for the city to consult. It is unclear, however, how much machine-readable disaggregated data will be made available to the transport authority, especially on vehicle speeds and speeding.

Active safety systems in vehicles include anti-lock braking (ABS), electronic stability control (ESP) and autonomous emergency braking (AEB). The engagement of such systems is some of the simplest yet most relevant surrogate safety data a vehicle can produce. Transmission of aggregated statistics to the cloud is not time-critical and occupies a negligible bandwidth. If all connected vehicles reported such events in a machine-readable, open manner, precious data would suddenly become widely available.

Vehicles' OBD ports certainly could signal ABS/ESP/AEB activations to third-party apps monitoring driving conditions, be it for insurance, coaching or other purposes. Wireless connections to users' smartphones should be considered as a possible evolution in OBD. With the observed integration of smartphones in the car entertainment system, requiring the broadcast of essential vehicle data to a smartphone would not be technologically challenging. If such a broadcast is designed as a one-way protocol, the risk of hacking would be eliminated.

Figure 6. See.Sense crowd-sourced road surface defect mapping

\section{CLUSTER MAPS OF ROUGH ROADS}

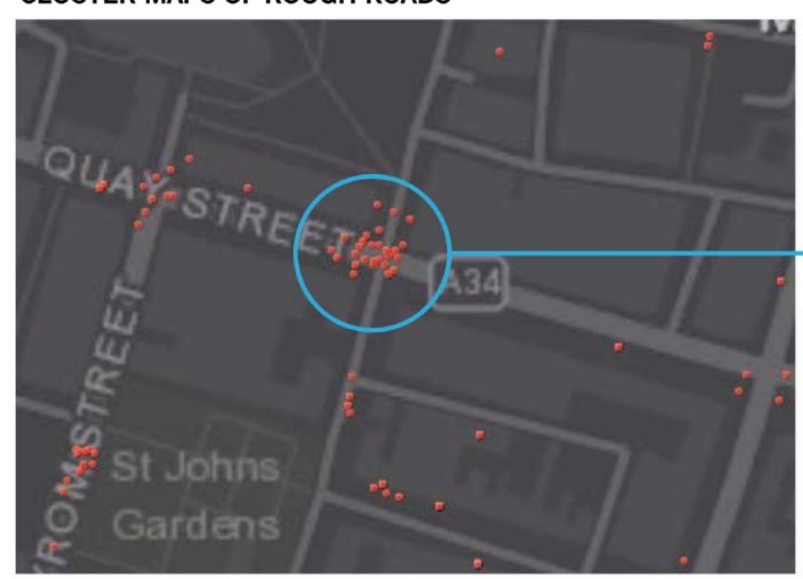

CORRELATION WITH POTHOLES

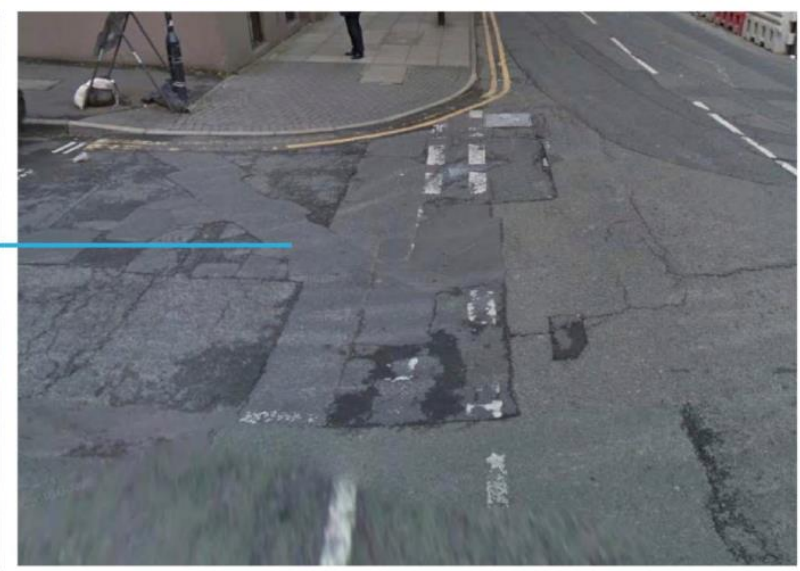

Source: Li and McAleese (2018).

Potholes are seen by some as safety-neutral in the sense that they slow down traffic and thus prevent a number of deaths and serious injuries. However, this idea should be challenged in areas where potholes might cause pedestrian injuries and where two-wheelers risk swerving or falling in the path of nearby vehicles. The uptake of cycling and micromobility places a greater emphasis on road surface quality, and requires more formal traffic calming solutions. To collect information on potholes and other cycling data, the City of Manchester worked in partnership with a smart bike light manufacturer. Sensors on the device enabled 180 cyclists to seamlessly report the exact location of the rough surfaces they encountered over a five-month period (Figure 6).

Curb activity is growing in intensity with the rise of ride-sourcing companies and food and parcel delivery. Curb activity is likely to contribute to degraded safety conditions, resulting from a lack of available bays and the subsequent occupation of bicycle lanes or pedestrian crossings. Specifically, increased curb usage produces a higher likelihood of "dooring incidents", potentially fatal collisions whereby a car door opens suddenly into the path of a person cycling (ITF, 2018e). For this reason, data on passenger pick-up and 
drop-off is extremely valuable to transport planners seeking to implement a Safe System approach (Figure 7).

Figure 7. Passenger pick-up and drop-off locations provided by ride-sourcing companies

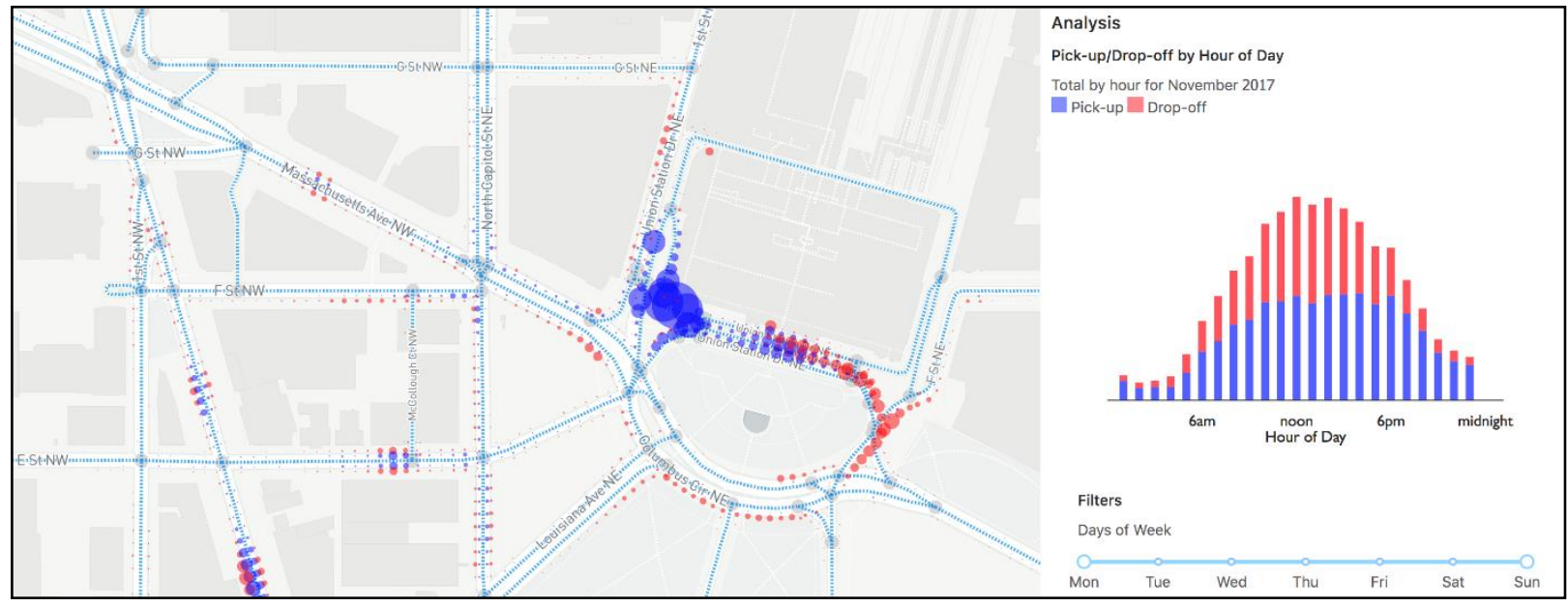

Source: adapted from SharedStreets (n.d.).

\section{How to map and monitor speeding}

Speed certainly is the most critical factor in crashes and also determines the severity of outcomes. Policy makers are encouraged to review their speed limits and to dedicate resources to enforcement. Yet, without an independent and non-intrusive speed survey solution, authorities lack:

- $\quad$ the intelligence to allocate their traffic calming efforts (be it enforcement, education or engineering) where they are most needed

- $\quad$ the ways to measure the effectiveness of their interventions.

An independent and non-intrusive speed data collection protocol should neither be visible to the road user on the spot nor result in penalties which would disclose sensor location.

For the analysis of the speeds and of speeding behaviour on the entire road network, it seems natural to envisage the use of floating car data (FCD). Transport planners are accustomed to procuring FCD in order to monitor congestion and refine traffic models. The technique most often consists in collecting journey time information by tracing vehicles through navigation apps or from re-identification. Traffic safety analysis is often based, opportunistically, on such datasets designed for transport planning applications. However, for a sound traffic safety analysis, FCD procurement should include additional requirements:

- $\quad$ it should avoid averaging speed across long sections of road, as doing so would combine queuing and free flowing traffic, therefore losing the information on the top speeds which may be achieved mid-block

- $\quad$ it should also avoid averaging speeds across multiple vehicles, as doing so would combine slow and fast vehicles, therefore losing information on the true speed of the fastest vehicles.

For these reasons, traditional transport planning floating car data - averaged over entire links and averaged over multiple vehicles - is of limited value to road safety practitioners.

The requirement of road safety professionals is to collect data from vehicles at a detailed spatial level ideally 10 - to 50-metre granularity in urban environments - so to measure the quasi-spot speed of 
vehicles. A ten-metre granularity is adopted by the SharedStreets platform collecting passenger pick-up and drop-off.

The question of spatial granularity is technical in nature. Granularity may have been limited in the past by lower data processing capabilities, and the effect of buildings in urban areas on GPS signal quality. Those issues are less relevant today with hybrid location-sensing techniques leveraging Wi-Fi or cellular tower locations in addition to GPS, but also with new Global Navigation Satellite Systems (GNSS) solutions coming to market (GSA, 2016). It may alternatively be solved by using vehicle odometers or smartphone accelerometers as part of a dead-reckoning approach, whereby position is estimated from earlier known positions and best estimates of speed and course (GSA, 2018).

The requirement of road safety professionals to collect the speed of each and every vehicle inevitably raises privacy questions which seem to delay the development of such data exchanges. Privacy protection could be ensured with mathematical solutions, using speed percentiles to replace micro-level data points, or by using robust de-identification methods (ITF, 2015).

Figure 8. High spatial resolution speed map using data from Dash

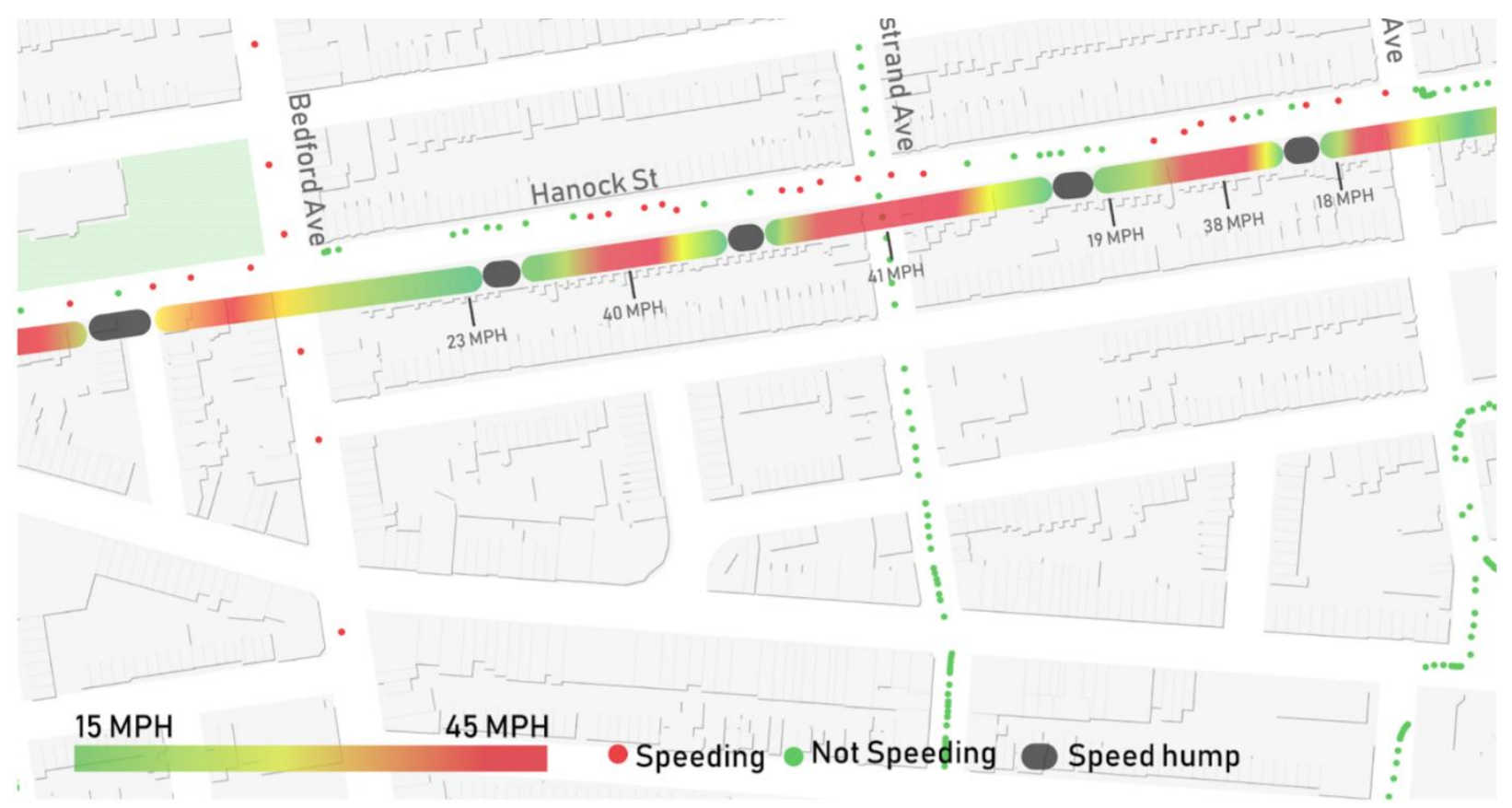

Source: Daud et al. (2018).

Floating car data will naturally rely on a subset of the national vehicle fleet. This subset must be considered a biased sample unless representativeness is proven. Data providers and customers should seek to build speed analysis on the widest possible and most diverse sample of road users. Nonetheless, for mapping and monitoring purposes, a sampling bias is often found to be acceptable.

Fleet size will be criteria for success. Some relevant applications become possible where data is collected from $2 \%$ of the general traffic, according to some estimates. This would enable the analysis of speed by month and by hour of the day on all roads with 1000 or more vehicles per day, with about 25 observations per time period on low-traffic roads. Authorities procuring such data should request that sample size be disclosed for each and every speed analysis segment. 


\section{Exposure data}

Floating vehicle data reveals occurrences of close calls or potholes, among other things, under the assumption that the instrumented fleet is representative of the entire fleet. If a smartphone app was distributed to identify potholes but entire districts were left unchartered due to the low uptake of the app by some population groups, the whole road surfacing strategy could be misled in obviously inequitable ways.

A possible solution might involve normalising the occurrence of events by the traffic volume of the instrumented fleet from which events are reported. To do so without adding new privacy protection concerns, several de-identification techniques could be envisaged. Since the removal of direct identifiers is not always sufficient to appropriately de-identify the information (OIC, 2019), privacy protection often requires the alteration of original data, leading to losses in accuracy, precision and general utility for analysis purposes (Yin et al., 2015). The trade-off between privacy and utility can be mitigated where analysts can point to areas where de-identification techniques can be applied which preserve the value of aggregate results.

De-identified data, once aggregated over time, provides adequate exposure data without disclosing precise vehicle activity. For instance, one could envisage a combination of data perturbation and data sampling, techniques which are part of a wider de-identification tool box (Box 17). Vehicles could communicate their exact location at random time intervals, say 10 minutes apart on average, and could also provide time stamps which are randomly altered by a few minutes. As long as the position sampling interval is much smaller than the incident re-occurrence interval, aggregate exposure data will be good enough to support the analysis. As long as the typical time stamp degradation offset is below 10 minutes, risk analysis could be disaggregated by time of day. If this is proven to resist re-identification attacks, an industry-wide standard in this area would be relevant.

General traffic information has long been essential to road safety professionals in the planning of interventions but also to the monitoring and understanding of risk levels. Traffic information includes pedestrian activity and that of all other road users, regardless of whether they contribute to any data collection exercise. Such information, called exposure data, contributes to understanding exposure to risk.

Pedestrians and cyclists have long been neglected in both street design and data collection. As a result, whereas motor traffic flows are modelled and monitored at least in some areas, analogous data for pedestrian and cycle flows are generally unknown in many cities.

A range of apps now collect cycling activity: Strava, GeoVelo, Bike Citizens, Tattoo and Trace, to name a few. Other mapping solutions, such as Google Maps, are also providing cycling navigation and can collect trace details. In addition, a number of other apps and mobile phone operating systems collect location data from which the mode of travel may be inferred, even when the apps or operating systems are not actively used.

Exposure data from all these platforms should, however, be treated with caution:

- $\quad$ Biases in user samples would lead to biases in the resulting exposure map. Biases could be based on gender, age, social and cultural groups, etc. Neighbourhoods with a low app use would appear as low-cycling neighbourhoods. Routes which are preferred by app users will appear more popular than they really are in comparison with other routes.

- $\quad$ The user sample varies over time in a way which may correlate with recent bicycle/scooter purchases or spring-time fitness resolutions rather than with true on-street activity.

- $\quad$ Some fitness apps would have a bias towards commuting journeys, while some navigation apps would have a bias against routine commuter journeys. This could result in negligible errors in 
mixed-use neighbourhoods, but cause problems where the zoning system separates jobs from housing.

In earlier research by the ITF (2015), the biases resulting from volunteered location data were illustrated. Strava, for instance, is an app that started as a way for cyclists and runners to compete against each other's times on defined segments. It has amassed a very large data set of geolocalised tracks around the world based on self-reported bike rides and runs by app users. It markets this data (under the Strava Metro product) to planners and city authorities to help shape urban transport policy. Strava claims it is capable of separating commuting tracks from leisure tracks. This doesn't, however, resolve a range of potential biases due to unclear representativeness in the user base. Given the app's stated purpose, a bias may emerge due to the type of bicycle commuter most likely to use the Strava app (e.g. more competitive, longer-distance commuters). This may result in a distorted vision of cycling route preferences.

A solution to address those biases is being explored by Bike Citizens. Their "calibration feature" correlates the GPS cycling data tracked by the app with the data derived from municipal bicycle counting stations. Calibrating recorded tracks with stationary counts allows GPS cycling data to be extrapolated and compared with overall road use. The solution seems particularly relevant but requires a large number of counting stations to address all the biases listed above.

In some instances cities have developed their own apps for the recording of bike traces (Mon RésoVélo in Montreal, ORCycle in Portland). In such cases, the cities must not only invest in developing an app, but also in promoting it to residents. Economies of scale could be envisaged if cities could rely on existing opensource standard apps or market leading solutions for cycling trace data collection, ensuring the best possible platform is used and the sampling bias is minimised.

The use of street imagery, drones and satellites will be discussed later in this report. Those solutions have the benefit of solving the sampling bias issue associated with voluntary data collection platforms. Street imagery is particularly beneficial in this area: not only does it collect pedestrian and cycling information, which satellites would struggle to do, but it can also report on gender.

\section{Infrastructure inspection and predictive maintenance through mobile sensors and imagery}

Infrastructure network screening consists of the examination of an entire infrastructure network to identify sites or road sections where further diagnosis may be relevant. Examples of such sites would be those where design or operations create a higher risk of fatal or serious injury and where there exists potential for risk reduction (Stipancic et al., 2018).

According to WHO (2018), 114 countries currently undertake some form of systematic assessment or star rating of existing roads. Road assessment programmes, such as the well-known International Road Assessment Programme (iRAP), offer a solution for the screening of entire road networks. By working in a systematic and comparable manner, and on a global scale, iRAP offers valuable information to better plan, prioritise and target road safety investments.

Such an assessment is now facilitated by the use of street imagery. IRAP has completed the survey and rating of 940000 kilometres of roads across 54 countries, often relying on camera images collected by dedicated camera-fitted vehicles. IRAP increasingly analyses data which is collected for other purposes. For example, it may analyse data that has been collected as part of road asset maintenance surveys.

Historically, network screening has been limited by two bottlenecks. One is the lack of resources to drive probe vehicles throughout the network. Another is the lack of time to analyse an immense quantity of data collected along the network. Partnerships and technology are now lifting both barriers. 
A complete assessment of infrastructure safety can involve the collection of very specific data on road surface skid resistance and therefore require the use of dedicated instrumented probe vehicles. However, the analysis of street images is at the heart of network screening efforts and could form the core of a simplified approach.

Screening a whole network from street imagery alone no longer requires instrumented probe vehicles, as street imagery datasets already exist for other purposes. Some such datasets are led by the mapping industry (Google StreetView, TomTom and HERE). Others are open platforms, such as Mapillary. Future imagery data sources may be formed by increasingly instrumented road users or vehicles choosing to contribute voluntarily and whose trip destinations have convenient broadband connections. Such crowdsourcing could involve members of the public, the automotive industry, e-scooter operators and public transit operators. A diversified source of images could complement image banks from the mapping industry, revealing night-time lighting condition. Images will be most useful if they are of sufficient quality, offer a wide angle and means of distance calibration to measure distance to off-road hazards.

\section{Figure 9. Computer vision applied to street imagery}

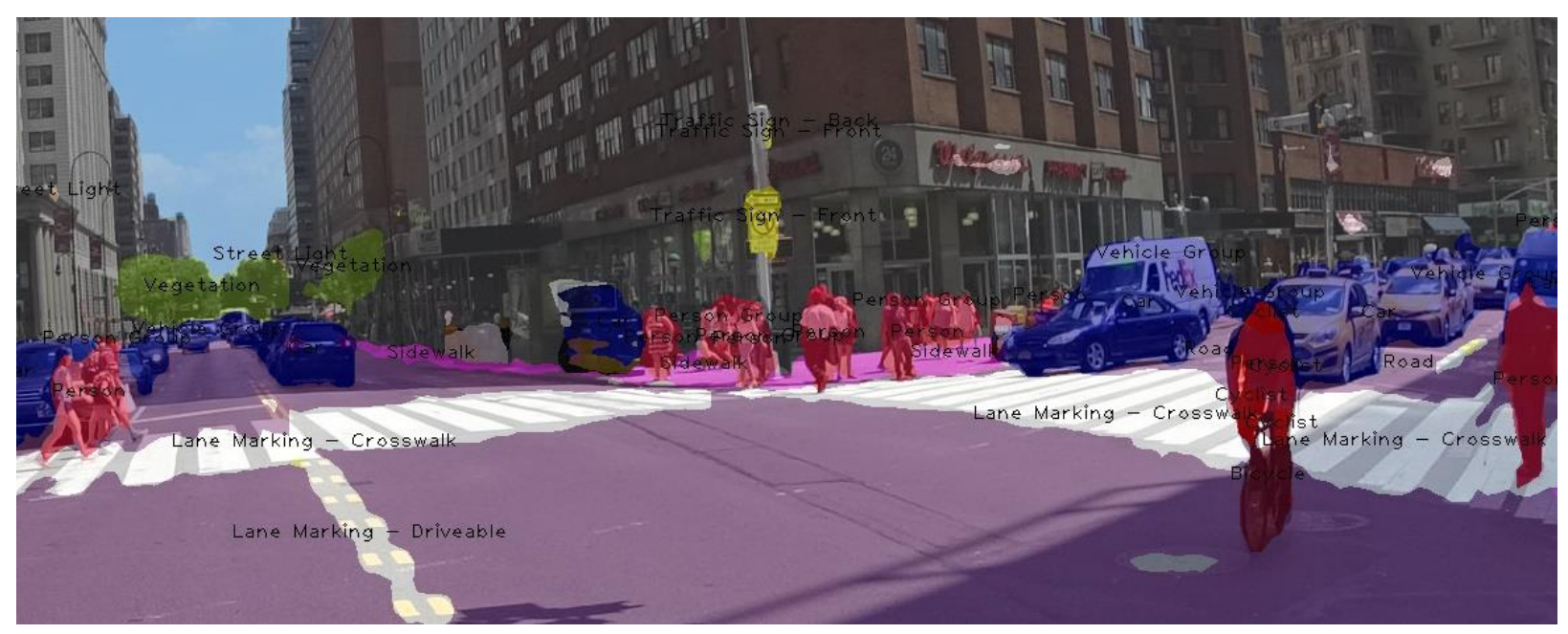

Source: Kontschieder (2017).

Artificial intelligence is another game changer which is boosting network screening capabilities. Using machine learning techniques, computer vision solutions exist to make sense of street imagery. Automating the analysis of images is the key to a much cheaper and faster road safety assessment programme.

Street imagery applications in the area of network maintenance include crash barrier repairs, vegetation maintenance (especially where signs are masked), horizontal and vertical signage maintenance, etc. Images can be used not only for asset inventory but also for asset condition (Immergis, 2016).

Railway tracks and train operators anticipate significant benefits from connected sensing capabilities delivered by IoT solutions. Having installed accelerometers on railway switches, a fragile and safety critical piece of equipment, the French rail network operator SNCF Réseau observed a 30\% drop in incidents along a high-speed line. However, this raises the question of the cost of instrumenting a whole railway network. 


\section{Box 9. Vaccines for Roads assesses and maps road safety problems}

If more than $75 \%$ of travel happens on three-star roads or better by 2030 an estimated 467000 lives will be saved every year worldwide. What's more, 100 million deaths and serious injuries will be avoided over the 20-year life of road treatments.

Vaccines for Roads IV, iRAP's new Big Data Tool, provides for the first time a health check on how safe the world's roads are, the projected human life and economic savings to be gained if countries meet the new UN Global Road Safety Targets, and case studies of success. The Tool, launched in 2018, unlocks insights into the world's largest road infrastructure safety database, summarising star rating and investment plan data - over 400 million data points, with more data integrated each year - based on 358000 kilometres of roads across 54 countries covering over 700 billion vehicle kilometres of travel.

Figure 10. Street with one-star iRAP pedestrian rating

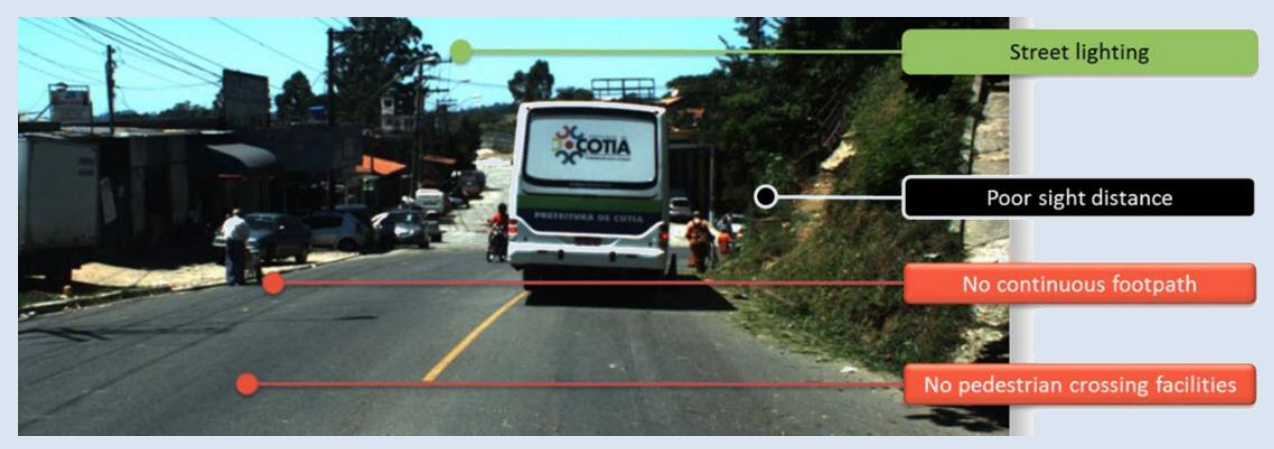

Source: iRAP (2018).

As a complementary approach, trains should also be able to report on potentially dangerous anomalies on the network, and contribute to predictive maintenance efforts. SNCF Réseau operates a train which is dedicated to network inspection. Industrial manufacturing company Siemens is working on inspection solutions whereby regular trains would play a role (Box 10).

\section{Box 10. Safety for rail transport: Siemens Mobility and Strukton Rail's Video Track Inspector}

Rail transport is made more efficient, reliable and safe thanks to condition-based monitoring, data analytics and predictive maintenance based on artificial intelligence. Video Track Inspector - a joint development between Siemens Mobility and Strukton Rail - is one example of how digitalised condition monitoring can optimise the maintenance of rail systems and prevent accidents.

Visual inspection of railway infrastructure elements is typically a slow and dangerous task, performed manually by track workers or special measurement trains. Video Track Inspector makes this process safer, combining data analytics and artificial intelligence knowledge from Siemens with the operational maintenance expertise of Strukton.

Video Track Inspector allows for the automated fault inspection of so-called insulated rail joints: essential safetycritical components in track circuitries that control signalling and prevent the train from derailing. Video cameras are installed below regular operation trains and deliver input for data analytics: at first, the rail joints are automatically identified; secondly, typical failures are identified and classified by an algorithm. On this basis, work orders for preventive maintenance can be automatically created and transferred to a maintenance management system.

Video Track Inspector presents numerous benefits: the automated fault detection allows savings for network operators, as well as higher reliability of fault detection due to exclusion of human error. Furthermore, Video Track 
Inspector eliminates foot patrols by track workers and the safety issues they face. In addition, because regularly operating trains are equipped with data-collecting cameras track closures are no longer necessary and are freed for regular operation trains and higher passenger comfort.

Source: Siemens (n.d.).

Public transport and other municipal fleets could be instrumented in reporting on road surface condition and hard deceleration. Going forward, one could envisage that mobile mapping systems are installed on municipal fleets, combining LIDAR and video equipment, so that quality data is collected and updated as part of routine vehicle movements. Mobile laser scanning could be used for the inventory of road markings and the monitoring of damage caused by wear and tear (Chhatkuli et al., 2017).

\section{A growing role for drones and satellites}

This section outlines several use cases for drones in the realm of transport safety, and touches on the challenges which will need to be addressed if such applications are to be fully unlocked. One of these relates to the safety of drone use itself - there are very real technical challenges surrounding the safety of drone use at scale - especially as concerns the risks of equipment failures, crashes and falling debris.

Collecting data from the sky will increasingly involve drones. In comparison to cameras located on streetlight masts, drones can be easier to deploy, offer more flexibility, a higher range of vision and a better estimation of vehicle trajectories and cinematics. This use case would involve what is referred to as support drones, as opposed to passenger drones and freight drones.

Three main applications for drones in transport safety are:

- Infrastructure inspection: either for predictive maintenance (searching for cracks, pot holes, structural damage before they become serious threats) or for general mapping and inventory purposes, which could be an input into safety audits. The strength of drones lies in potential cost savings and improved data quality compared to other methods. Drones, and especially autonomous drones, could survey a large network more flexibly and cheaply than a ground vehicle having to navigate a congested network.

- Traffic surveys: recording the flows and trajectories of vehicles and pedestrians, vehicle speeds, and close call incidents for limited survey zones, like in junctions. A disaggregation of data by road user type is now possible through computer vision and adds much value to the survey. Traffic surveys typically last over several days if not weeks, with the goal of capturing different flow patterns on different days of the week and at different times of day and night.

- Enforcement: for the rapid and flexible deployment of enforcement capabilities on all network types. Such a covert and all-encompassing enforcement solution could help governments reduce the number of people killed and seriously injured on the roads by filling the geographical gaps between fixed enforcement systems (as speed cameras on board unmarked floating vehicles currently do) but also by having the flexibility to control a greater diversity of offences than fixed equipment. In countries where helicopters are deployed for traffic safety purposes, a conversion to drones would deliver precious savings.

Until a dramatic change in battery technology occurs, support drones will have significant constraints on flight time. Three solutions will most likely be explored for transport data collection:

- A drone could hover for a limited duration, typically 30 minutes, then return to a base where its battery is swapped. A similar solution would be the use of fast-charging stations, either deployed in ad-hoc locations by the survey team, on the roof of a survey land vehicle, or integrated into the 
urban environment (streetlight heads, rooftop of bus shelters, etc.). This would cause interruptions in the data collection.

- A drone could be tethered to a power source with a power cable, enabling the continuous collection of data, but limiting its altitude and, therefore, its vision.

- Balloons could be used to provide a lift to keep a drone in the air longer. Such drones are often called blimps. They emit a much lower level of noise pollution but leave a greater visual footprint.

The most relevant solution for the continuous use of support drones - between fast-charging, tethering and blimps - will naturally depend on the survey environment. Noise pollution remains a major barrier to the use of drones. From a data collection perspective, it should be noted that interruptions in the recording is rarely a major issue. If the goal is to count road users or measure the frequency of close calls, recording 30 minutes in every hour would be acceptable. The loss of data can be compensated by an extension of the survey campaign duration, from, say, one week to two.

The use of drones raises more than just technical challenges. Regulatory issues also accompany any discussion of drone use. While a fixed camera can be left recording information unattended for an unlimited amount of time, collecting transport data from drones over a long period is more complex. Several countries require that a drone remains in the line of sight of a dedicated pilot at all times. In those conditions, it makes no economic sense to use drones for surveys requiring a long observation period on a single location, something which fixed cameras will do for a fraction of the cost.

Developing social acceptance for drones will be vital for their use in transport safety applications. Regulations in favour of medical applications of unmanned flights would likely foster public support for the technology. These applications include the transport of blood and lab samples. On the other hand, the use of drones to enforce traffic rules, however legitimate, could compromise public support for drones.

Drones also raise privacy protection challenges as they are technically capable of recording images from within private properties, at an angle from which building occupants do not expect to be watched.

There are significant barriers to the development of drones in urban areas. These include the risk of falls, noise pollution and privacy issues. Addressing the many challenges in transport safety in human settlements is crutial to building public acceptance for the use of drones.

\section{Satellite data}

Satellites images offer obvious economies of scale in the analysis of network configuration and network usage, particularly in the road sector where information is scarce.

Researchers analyse satellite images to:

- identify junction layout (Barsi and Heipke, 2004)

- map driveways and other private access routes to the road network

- estimate traffic volume by vehicle type (Leitloff, Hinz and Stilla, 2010; Larsen, Salberg and Eikvil, 2013; Gao et al., 2014).

Use of satellite imagery has been limited due to a lack of video data. Photographic images do not allow for parked vehicles to be distinguished from moving vehicles. They do not provide speed estimates. Video sequences that chart movement would facilitate road safety research (EarthNow, 2018) and particularly benefit road assessment programmes. Satellites are also not very suited to detect pedestrians and cyclists.

Satellite and drone data are being considered for the mapping of a winter phenomenon known as "sneckdown", where vehicle tracks in fresh snow indicate real use of road space and often reveals large, 
underused, parts of the roadway that could be reallocated to other uses by, for example, creating wider pavements, shortening pedestrian crossings and calming traffic.

\section{User feedback on infrastructure faults and incidents}

In the age of information technology, collecting active feedback from road users has become easier. Smartphones provide accurate location and can take pictures to document potential issues. Apps specifically developed for cyclists, like PING if you care!, See.Sense Icon, and ORCycle, allow cyclists to report dangerous locations. Solutions could also be developed allowing children to report problems along their route to school.

One could argue that such technologies allow people to report things they perceive as unsafe, regardless of the real crash risk. However, this should not be seen as a weakness. The perception of safety is a fundamental goal for policy makers working to create a liveable environment where people of all abilities feel they may move about safely.

A much greater weakness of such apps is the lack of representativeness of their user pool. In the population using self-reporting apps, any bias towards a given social group and associated neighbourhoods may result in a biased mapping of road safety priorities and risks diverting investments away from where they are most needed.

ITF (2016c) reported that Smartphone penetration rates are not uniform around the world, or even within countries or cities. As ubiquitous as location services are, with almost complete coverage for those with a GPS-enabled mobile device, the data generated is still just a sample of the whole population. Sampling bias is further exacerbated by different penetration rates for specific apps and smartphone operating systems. Transport authorities must carefully consider issues with non-representative sampling when looking to use this type of data.

Biases in the user base can be addressed by controlling for the underlying number of potential contributors who have travelled along a given street where a report is logged. This is the strength of the PING if you care! initiative, where street danger reporting is associated with the collection of cycle traces.

One way to broaden and retain the user base of such apps would be to design a single app to report a wide range of street problems, including cleanliness, lighting, drainage, street furniture and parks as well as safety concerns. In Paris, the faults-reporting smartphone app DansMaRue adopts an even broader scope which goes beyond streets and parks to include municipal swimming pools.

Another way to encourage members of the public to report issues through apps would be to simplify the process by offering a single interface. Municipal boundaries are not always obvious to the population, yet people cross such boundaries on a daily basis. In this context, users would appreciate using a single app regardless of where they are, instead of having to question which local government is responsible for the street fault the user wishes to report. Even where people are aware of the municipality they are travelling through, reports should be directed to different authorities depending on the level of road hierarchy. Not everyone is aware of this. The FixMyStreet Platform is an open-source project that responds to this need, helping to create websites for reporting common street problems to the appropriate authority. It has been running in the United Kingdom since 2007 and receives thousands of reports each week.

Platforms for reporting street faults should be transparent, showing the status of earlier reports made by the entire community of users. Not only does it show users they are not wasting time with duplicate reporting, but it also holds the road authority accountable, an aspect which is especially important for safety critical reports. Transparency, therefore, places an incentive on authorities to resolve issues more quickly, which, in turn, is an incentive for members of the public to use the platform. An additional 
advantage for governments is that information from user reports can be used as evidence to support road safety interventions.

\section{Box 11. Vision Zero Boston Safety Concerns Map}

As part of a Vision Zero agenda, the city of Boston has launched an online mapping tool for members of the public to report safety concerns. The tool is called the Vision Zero Boston Safety Concerns Map. Reports are classified along the following categories:

people are not given enough time to cross the street

people have to wait too long for the "Walk" signal

people have to cross too many lanes / too far

people cross away from the crosswalks

people run red lights / stop signs

people speed

people double park their vehicles

people don't yield while going straight people don't yield while turning

it's hard for people to see each other

there are no sidewalks or they need maintenance

there are no bike facilities or they need maintenance the roadway surface needs maintenance reporting of something that is not listed here

In the first six months after the map was launched, more than 11000 citizen's reports were received. The map also indicates the areas with the highest concentration of crashes, and allows residents to view crashes sorted by type - pedestrian, bicycle, or motor vehicle. (Bosco, 2017)

Source: https://www.boston.gov/transportation/vision-zero\#input-and-data.

Social media is another source of information for road authorities to mine and identify problems. There is, however, considerable uncertainty in the interpretation of social media posts where geolocation and images can be missing. The question must be asked, then, why do people turn to social media to report street defects? Is it because they mistrust official reporting apps that lack public logs of reports made by the community at large? Are they seeking transparency and accountability on social media that they have been unable to find on official channels? Such questions should be investigated and addressed.

\section{Users reporting issues through video evidence}

In Israel, a system called "Safe Lane" empowers drivers to report severe driving infringements in real time (ITF, 2017). Videos recorded by volunteers go through a screening process. Cases that are serious enough and match police criteria are passed to the police for review and enforcement. Also called "Guardians of the Road - Social Change on the Roads", the system results from a joint venture between the National Road Safety Authority, Israel Police Traffic Department and the NGO Nativ Batuach. The goal is to decrease the number of traffic-related casualties through the use of innovative technologies and civic involvement.

In Wales, as part of "Operation Snap", the police collect camera footage from all roads users - from pedestrians to cyclists, motorcyclists, horse riders and drivers of all vehicles (Road Safety GB, 2018). Pioneered by the North Wales Police (2016), the operation simplifies the evidence submission process so to encourage greater public participation. The operation is believed to be an important step towards changing behaviours of road users who become increasingly aware that they are being watched, but also of road users equipped with cameras.

A similar behaviour change in the presence of camera equipment was reported by Ariel et al. (2016) in a different context. The authors reported that when police were equipped with body-worn cameras the 
behaviour of both officers and citizens improved, a phenomenon they call "contagious accountability", and fewer complaints were made against the police.

When developing the use of camera footage, the road safety community should bear in mind the danger of road users getting distracted by their equipment while on the road. Users should be encouraged to report events in off-traffic situations. Camera equipment should facilitate this by offering a single command which marks the time of the event for the recording to be used at a later stage.

One could argue that video footage, like all user reports, are neither uniform nor systematic. The penetration of dash cams and body-worn cameras in the general traffic is nonetheless beneficial in changing the behaviour of all road users. Evidence is reviewed by police teams as if they were witnessing the situation while on patrol, ensuring fair treatment for all road users.

If authorities do not accept video footage they run the risk of misleading the general public to believing that traffic offences are not a serious matter. Behaviours would deteriorate in a similar way as has been observed when and where traffic police numbers drop (Rezapour, Wulff and Ksaibati, 2018).

Close passing and failure to yield

Close passing is a dangerous and intimidating situation where the driver of a motor vehicle overtakes a cyclist at close range. Police in the United Kingdom attribute "passing too close to the cyclist" as a contributory factor in one in four serious collisions between cyclists and large vehicles (Knowles et al., 2009). DfT (2017) states that 59\% of non-cyclists in Britain feel that it is "too dangerous" for them to cycle on roads. When urban motorists close pass, fail to yield at pedestrian crossings or turn from larger roads into side roads in close range of pedestrians and cyclists they put those road users at risk. In rural areas without footpaths, intimidating close passing manoeuvres are frequent. Some communities are putting measures in place to minimise such dangerous behaviour.

In Britain, the West Midlands Police proactively target close pass drivers who endanger riders. Bike patrol officers radio reports of close pass drivers to in-car patrols who stop the infringing drivers. In addition, officers make online reports based on video footage of close pass drivers obtained from dashboard cameras and cycle-mounted cameras. The same footage allows them to monitor dangerous or careless driving and mobile phone use while driving.

Under the project Radmesser in Berlin, 100 volunteers have equipped their bicycles with sensors to measure passing distance (Tagesspiegel, 2018). Results reveal that the intensity of close passes varies according to the time of day and by district and that slower cyclists experience a higher number of close passing events per unit distance travelled. This finding would justify giving higher priority to street design and the physical protection of cycle routes on the grounds of inclusiveness. Radmesser data could also be used by local authorities to identify communities that would benefit from targeted enforcement or education campaigns.

Data collection empowers people to raise an issue otherwise neglected as non-measurable. Video analytics and fixed roadside equipment mentioned earlier in this report could be combined with portable solutions in both rural and urban areas to fully cover all parts of the road network. 


\section{Focusing on individuals and their behaviours}

This section reports on solutions where data is collected to improve driver behaviour. This could be through enforcement, education, nudges, alerts.

Enforcement efforts have diminished in some countries as police resources have been re-allocated to other duties. A data-driven approach to enforcement, whereby high-risk situations are revealed and focused on, could compensate, at least in part, for a fall in resources. Enforcement is one of the cornerstones of road safety, yet it raises recurring questions of acceptability. Governments could encourage the public's acceptance of enforcement by allocating enforcement revenue towards road safety budgets and victim support. Road users would possibly look more favourably on enforcement if it targeted high-risk situations and behaviours. Data could help enforcement concentrate on places and times of day when most offences are observed, leading the public to believe that data is used to maximise revenue. There is therefore a fine line to follow on the route towards data-driven enforcement.

Enforcement and education are often seen as an either/or choice to be made, whereas the two approaches actually complement each other and should be developed jointly. Education without enforcement rarely works, as the lack of enforcement is interpreted as a lack of government interest in an issue. Enforcement without education campaigns leads the population to believe that the government is taking advantage of a minor issue to raise revenue through infraction fines.

Education campaigns benefit from data collection and analysis. With access to relevant databases, researchers can develop a detailed socio-demographic profile of individuals involved in specific crash types. Behaviour change campaigns can then be planned according to localised needs. In the United Kingdom, Ursachi et al. (2018) mined the police crash database for drink-drivers involved in collisions, and matched their home postcodes with a geodemographic segmentation system called Experian Mosaic. Not only did the analysis reveal that drink-related crashes are more common for certain driver groups than others, it also linked the results to a consumer lifestyle segmentation system. The system offers insights on how to reach these groups through marketing channels: specific newspapers or magazines, websites or television channels, etc. With such results, it is possible to design targeted drink-drive compliance campaigns. Advertisement can be targeted, leading to substantial cost savings.

\section{Driver coaching}

Every driver may develop bad habits over time, especially on familiar roads, increasing the probability of crashes. Flagging risky behaviour requires data collection. Offering relevant feedback to drivers requires more: it involves advanced big data analytics.

Driver coaching has the potential to deliver a noticeable, large scale behavioural change. Fleet managers who have implemented driver coaching in the workplace have seen the added benefit of cut insurance costs. Coaching typically involves ad hoc telematics devices or smartphone apps or a combination of both, and records vehicle dynamics: longitudinal and lateral accelerations, speed, etc.

Data collection for traffic safety coaching could equally benefit eco-driving targets. In both fields, similar behaviours deliver best outcomes. It should, however, be noted that vehicle dynamics are not only determined by driver behaviour. External factors such as road layout, signal timing and congestion also play a role. For this reason, driver coaching solutions should involve a thorough statistical work, building on a vast sample of drivers. 
Gathering data from a vast number of drivers requires connectivity. To avoid strain on user's cellular data plans, users could restrict data transfer to times when a Wi-Fi connection is available, such as in the workplace or at home.

Smartphones are gaining popularity in driver coaching applications:

- Smartphones have dual-facing built-in cameras. Road-facing camera images could estimate the distance from the vehicle ahead, while driver-facing cameras could monitor distractions and drowsiness.

- Smartphones enable fleet operators to roll out a coaching solution to an entire fleet of vehicles, regardless of brand or model, without concerns of interoperability.

- Smartphones can capture vehicle dynamics from built-in sensors like accelerometers, gyroscopes and Global Navigation Satellite Systems (GNSS).

Vehicle position data from GNSS is unavailable in tunnels and severely degraded in urban canyons due to blocked lines of sight to satellites or multipath propagation. In such situations, a dead-reckoning approach could be envisaged, whereby position is estimated from earlier known positions and best estimates of speed and course. Vehicle manufacturers could use odometer and steering data to implement dead-reckoning, but smartphone app developers could implement it, as well, with accelerometer, compass, and proximity to known Wi-Fi access points.

Ultimately, driver coaching should be analysed along with crash data to establish evidence-based driving recommendations. The idea is to calibrate models capable of representing which behaviour would minimise crashes and claims. Such analysis would be greatly enhanced through partnerships between insurers and coaching product developers.

The future of driver coaching lies in personalised alerts, feedback and training. A voice interface is preferable for the driving task and would act like a personal assistant providing targeted recommendations.

\section{Sensing driver distraction and drowsiness}

While information technologies offer immense opportunities for transport safety, smartphones and dashboard display equipment can also be a visual distraction. As driving assistance systems and semiautomation develop, there is a risk that road users turn their attention to communication and entertainment devices and away from the road. A lack of evidence makes it currently impossible to ascertain the number of crashes in which such distractions play a central role.

Manipulating a phone when driving increases the risk of a fatal crash by $66 \%$, according to the AAA Foundation for Traffic Safety and the Virginia Tech Transportation Institute (Owens et al., 2018). In spite of this, a 2018 roadside survey noted that $3.4 \%$ of drivers in the US state of Virginia were seen using a cell phone (IIHS-HLDI, 2019a). The survey also revealed that drivers are talking on hand-held phones less but handling them more often than in recent years. Extrapolating from the survey results, several hundreds of fatalities a year in the United States could be attributed to drivers distracted by their phones.

The actual number of crashes which involve driver distraction remains unknown. This number can only be under-estimated in investigations relying on people truthfully telling law enforcement officers what they were doing or voluntarily handing over their phones for inspection. Road safety research in this area certainly needs event data recorder technology (EDR) capable of revealing driver distractions.

Phone manufacturers are offering some solutions - in the form of opt-in options - that limit distracting calls and notifications while retaining other functionality: to serve as a navigation system, for instance.

Smartphone blocker solutions use motion sensors and proximity to known network connections to detect 
driving. The blocker solutions can silence the phone, redirect incoming calls to voicemail or respond to text messages with a pre-programmed message.

The awareness and uptake of such functionalities remains insufficient, as a survey found that only one in five owners of eligible phones had their Do Not Disturb While Driving feature set to automatically activate when driving or when connected to their car's Bluetooth system. "Even though smartphone owners aren't rushing to use blocker apps, they do seem open to reminders nudging them to reconsider," says Ian Reagan, the study's co-author. "Periodic follow-up prompts from Apple might boost use of Do Not Disturb While Driving's automatic mode and help to curb at least one form of driver distraction." (IIHS-

HLDI, 2019b)

The automotive industry is making efforts to integrate smartphones with the cars' infotainment system, enabling the user to place hands-free calls, use voice commands to dictate messages or to hear voiced messages. While this sounds positive, as it allows drivers to keep their eyes on the road, it is nonetheless worrying in terms of users' mental workload and general distraction while driving.

For various reasons automatic and mandatory mobile phone signal suspension in moving vehicles has not been seriously considered. Not least among the reasons why is that phones in a moving vehicle could be operated by passengers and there would be no grounds for preventing such legitimate use.

Seat occupancy sensors, already deployed as part of seatbelt reminder systems, could detect when a driver is alone. In this case, the automotive and smartphone industries could cooperate to devise solutions preventing the excessive use of smartphones in a vehicle which indicates that the driver is alone in the vehicle. Such a system could turn the screen black when excessive eye contact is made with the screen while the vehicle is in motion or could allow full smartphone use in periods of automated driving. This would require communication between the vehicle and the phone regarding a number of variables: seat occupancy, motion, automation level.

When a driver is not alone in a vehicle, which is relatively rare, the problem is more difficult to solve. One can hope that peer pressure from vehicle occupants would regulate driver behaviour to some extent. Alternatively, facial scanners, if and when they are implemented on both smartphones and vehicles, could help smartphone apps distinguish the driver from the passenger. In this case, on a voluntary basis, software solutions could block incoming calls and notifications.

Driver distraction will only get worse in the foreseeable future. Smartphones, smart glasses and vehicle infotainment systems will compete for the driver's attention. There will be little commercial incentive for automated shut-down of those systems during the driving task, and as assisted driving progresses, users will increasingly expect to be entertained.

Solutions will need to be developed to protect road users in light of these technological evolutions. While technically feasible, solutions that allow the vehicle to track a driver's eye movements have not seemed commercially attractive so far. Regulators could require an alert system (similar to seatbelt reminders) to remind drivers about looking ahead. However, simpler solutions may be found.

Usage-based insurance contracts often come with a smartphone app, which has to be turned on every time a person drives. There is an obvious case for such apps to monitor or block incoming calls and reduce distractions. Smartphone apps developed by insurers could, therefore, play a key role in future road safety measures. They could not only block phone usage by the driver, but also monitor driver eye movements and prevent other distractions. Automotive and smartphone industries should combine their resources to jointly develop both practical solutions and awareness campaigns that encourage a cultural change where drivers benefit from the full range of new technologies in an environment that is safe for both themselves and others. 
Alcohol, drugs, painkillers and sleepiness

The consumption of alcohol, drugs and medicine, but also simple fatigue symptoms, all result in various forms of impaired driving which, similarly to distracted driving, increase the risk of fatal crashes. Whereas solutions can be developed independently to address on each individual cause of drowsiness (ethanol concentrations, cumulated driving hour regulations, etc.) comprehensive solutions should also be investgated. Analysing body signals - body temperature, heart rate, the motion of eyes and eyelids - would help detect the general state of a driver's drowsiness, regardless of the cause.

Drowsiness detection will likely develop in two strands:

- $\quad$ One solution could be based on vehicle sensors (Taub, 2017). Sensors placed in the driver seat could monitor vital signs, such as breathing and heart rate (Neteera Technologies, 2018; Cho et al., 2017; Vicente et al., 2016). Sensors facing the driver's eyes could monitor skin temperature and eye activity (Burke, 2018).

For instance, using an infrared camera above the steering wheel, the Citroën DS Driver Attention Monitoring system identifies any reduction in driver alertness (DS Automobiles, 2017). The system continuously monitors:

$\circ \quad$ the eyes for signs of tiredness (blinking)

- the face and head movements for signs of distraction

- the course steered by the car in its road lane (driver deviations or steering movements).

- Another solution could be based on smartphones, smart glasses, and other forms of personal health sensors. As new smartphones and wearables tend to be purchased much more frequently than vehicles, they could offer a more rapid technology solution than vehicle-based technology. This is even more important in countries where the majority of the vehicles on the road are secondhand vehicles from wealthier countries, and where innovations take decades before reaching a significant share of the fleet.

Some systems could monitor heart rates and body temperature as opposed to eye movements. Yet, where they involve eye scanners, drowsiness surveillance systems could offer the added benefit of distraction surveillance, offering a greater use case than other systems. Drowsiness and distraction are closely related, for they can both be revealed by eye movements (Jo et al. 2011). The European Commission (2018a) recommends the implementation of distraction recognition systems on new cars and explains that "Advanced Distraction Recognition may also cover drowsiness and attention detection".

\section{Monitoring the behaviour of professional drivers}

Close to $40 \%$ of all road deaths in the European Union are work-related, according to estimations conducted by Adminaite et al. (2017). The estimation naturally includes professional drivers (e.g. truck, bus or taxi drivers) and travellers (e.g. salespersons), commuters, people killed in crashes involving one of the user categories mentioned earlier, and people who routinely work on or near the road (e.g. road construction workers). The number of work-related road deaths can be identified in police reports when a fatal collision involves a heavy goods vehicle and bus, as it is uncommon to drive these vehicles for nonwork related purposes. However, in many countries the purpose of the journey of van and car occupants and vulnerable road users is not recorded by the police. This section is dedicated to the behaviour of professional drivers, in the context of bus, taxi, truck or other commercial fleet operations.

Businesses purchase a large share of vehicles in most markets. In France, for example, company purchases account for half of all vehicles sales (Lagarde, 2017). Large fleet operators, especially those carrying dangerous goods like oil and chemical products, have elaborated safety management systems and 
systematic approaches to embed the management of risk as a core business function (Box 12). However, large parts of the trucking industry rely on either owner-operator drivers or subcontracted drivers, a situation which is not conducive to safety management systems. This eco-system of small operators (up to $90 \%$ of truck drivers in Mexico or Korea are owner-operators) risks placing the responsibility for road safety squarely on the shoulders of the drivers (ITF, 2018b). Unless industry-wide interoperable standards are developed, it also raises significant barriers to the adoption of fleet telematics.

A first step to influencing the behaviour of professional drivers is documenting their behaviour. One way of doing so is through outcomes, such as crashes. Crashes are rare events at the individual level and do not adquately represent the behaviour of one particular driver. Crashes at an aggregate level, however, are useful in monitoring the performance of a bus company over time, or comparing the performance of different bus operators.

Surrogate safety metrics are therefore essential, and they can be presented in two groups:

- Qualitative: this can be reports by members of the public regarding a bus or ride-sourcing driver

- Quantitative: this can be occurrences of speeding or distracted driving

It is not clear that hard-braking events indicate risky behaviour. Indeed, one could argue that hard braking is a natural occurrence in the driving task, considering the unpredictable behaviour of other road users. A better solution would be to identify which hard-braking situations are caused by distraction (the driver's eyes leaving the road for a sustained duration) or by a poor choice of headway. Such systems are technically feasible using current technologies and could be fitted to vehicles or delivered through smartphone apps.

\section{Box 12. Five components of a safety culture}

Safety culture, as defined by Reason (1997), rests on five components, namely:

1. Informed culture - The organisation generates significant data on incidents and accidents, which are complemented by safety audits and surveys on safety environment

2. Reporting culture - Employees are encouraged to report their errors or near misses and take part in surveys on safety culture

3. Just culture - The establishment of a trust relationship between employees and employer, where reporting mistakes and incidents is encouraged and employees know they will be treated fairly if they make unintentional mistakes

4. Flexible culture - The organisation shows that it is able to adapt its practices when warranted

5. Learning culture - The organisation learns from incident reports, safety audits and internally reported issues, resulting in improved safety.

Inspired by those five components, a Safety Management System (SMS) requires a just culture within a company, where staff can report mistakes, omissions or incorrect decisions without the fear of disciplinary actions but where there will be no tolerance for gross negligence or unlawful acts. SMS efficiency also depends on the ability to collect data and use it in predictive analysis to foresee issues and manage them. However, the collection of this data may raise employee privacy concerns, which must be weighted against the safety benefits.

Source: ITF (2018b). 


\section{Intelligent long-distance road freight and safe truck drivers}

Truck operations are impacted by Cooperative Intelligent Transport Systems (C-ITS) and by the progressive automation of long-distance road freight. ITF (2019a) envisages driver assistance and automation technologies as a way to increase safety, via platooning, for instance. Platooning is a promising technology for long-distance road freight in which a number of trucks autonomously follow a truck ahead. It is likely to be an early application of C-ITS. It is also economically advantageous for the involved parties because it significantly reduces gas consumption. Furthermore, platooning is to be applied in an environment - mostly inter-urban highways - adapted to mature C-ITS technology. However, regulations and solutions to accurately notify non-connected road users of the presence of a platoon are still in progress.

That report also provides both sides of the debate on truck drivers' drowsiness in the context of SAE level three autonomous vehicle operations. SAE level three technology allows a driver not to concentrate on the driving task, so long as they are available to regain manual control of the vehicle at any time. Some stakeholders hold the view that drivers can be more attentive, and perfectly capable of regaining manual control at any time, if technology allows them to perform interesting secondary tasks, on a tablet computer, for instance. Other stakeholders provided evidence that distracted car drivers in autonomous driving conditions would display higher levels of drowsiness and would require a considerable amount of time to regain manual control.

If doubts exist on the safety of SAE level three technology, the rationale becomes stronger for a number of regulatory responses, essentially EDR and systems for drowsiness and distraction monitoring.

Industry experts and professionals have reported on the systematic use of on-boad computers in their truck operations. The use of telematics in professional vehicle fleets was described as easier in countries with lower levels of privacy protection regulations. For instance, while the capture of video images inside the truck cabin is developing in Africa, real or perceived regulatory barriers delay the development of such technology in other countries. This raises a particularly relevant challenge for regulators.

Ride-sourcing drivers in the gig economy

The gig economy involves independent workers contracted for short-term temporary missions. In the United States, some of the major employment sectors of the gig economy are the construction industry and professional and business services. While those have been stable over the last decade, transport services have been increasingly and rapidly affected by the gig economy (BLS, 2018). This includes the transport of persons and goods delivery. This shift is an international phenomenon. Large ride-sourcing and platformbased goods and food delivery companies, such as Uber, Didi Chuxing, or Lyft, play a major role in the industry. They are complemented by a legion of initiatives developed locally in emerging economies, such as Beetaxi in Cameroon, Careem in Egypt and SafeMotos in Rwanda. The safety of delivery and courier operations is not investigated in this section. They are, however, included in a recent survey from the United Kingdom by Christie and Ward (2018).

Ride-sourcing platforms cover most urban centres around the world, which means that the behaviour of drivers providing platform-mediated services has a widespread effect on road safety. Ride-sourcing suffers from a rather negative image in terms of road safety in some countries. In other countries, compared to informal micro-transit, or traditional taxis, ride-sourcing can be seen as the safer option. In the Philippines, for instance, a national regulatory framework imposes more rigorous driver vetting processes and vehicle safety inspections on the ride-sourcing industry compared to the traditional taxi industry. The framework imposes a unique identifier on each driver which is portable across platforms to track issues relating to multi-homing (ITF, 2016d).

Beyond publicised cases of drivers involved in crashes, the actual impact of ride-sourcing on road safety is still uncertain. Studies on that matter offer contradictory accounts (Brazil and Kirk, 2016; Barrios, 
Hocheberg and $\mathrm{Yi}, 2018)$. However, drivers fatigue has been recognised as a severe issue by city transit associations (NYPTA, 2017) and, more notably, by the American Academy of Sleep Medicine (Berneking et al., 2018).

Drivers' drowsiness can be explained by the fact that many drivers work on ride-sourcing platforms as a secondary revenue source, meaning that they often hold two jobs. The nature of drivers' remuneration schemes is another contributor to driver fatigue. Since drivers are paid according to the number of rides they complete, they may be compelled to drive fast and adopt more dangerous behaviours. Some ridesourcing platforms push this logic to the extreme, resulting in situations that could be compared to cities where bus drivers compete for passengers and routes because they are paid on this basis. Yet, ride sourcing platforms have created a number of incentives to encourage their drivers to adopt safe behaviour. Collecting user feedback is a widespread practice and allows platforms to spot dangerous drivers and take appropriate action.

In the past couple of years, ride-sourcing has also introduced a cap on working hours to address the issue of drivers' fatigue. For instance, Lyft shuts off its app after a driver has been online for 14 hours, whether those hours are consecutive or not. The app then doesn't let drivers back to work for the following six hours. In 2017, Uber updated its regulation in Chicago to prevent its drivers from working more than ten hours within a 24-hour period. This working cap is based on online time rather than driving time (Uber, 2017).

Platforms should consider which exact definition of "driving time" to apply. Drivers can simply log off the app for a short amount of time while they take a ride with a competing platform. Such behaviour is equally simple to detect, in comparison to a genuine break, since the driver would log in again in a different part of town. Such behaviour should neither be a solution to bypassing driving time caps, nor become a source of distraction for drivers constantly logging in and out. Further steps could be taken to prevent the gig economy sector from breaching the driving time restrictions applicable to other commercial drivers. ITF (2016d) suggests that regulators require a single, digital, driver ID number that allows for crossplatform tallying of cumulative driver hours. Christie and Ward (2018) similarly recommend that platforms share data on driving and riding time via the licence number of the drivers and riders with built-in alerts if time is exceeded on a separate digital platform.

\section{Contracting responsible bus operators}

London has made ambitious commitments regarding bus safety. Successive Mayor's Transport Strategy declarations set the target of a $70 \%$ reduction in the number of people killed or seriously injured on or by a bus by 2022 (against a 2005-2009 baseline) and zero people killed on or by a bus by 2030. This policy framework is supported by London's Bus Safety Programme, launched in February 2016. (TfL, 2018)

In the framework of this programme, Transport for London awarded funding to several Bus Safety Innovation trials, of which two are specifically powered by new sensors (TfL, 2017b):

- Collision Prevention: Installing a forward facing vision sensor to provide the driver with advance collision warnings and a speed limit indicator. This aims to reduce collisions with pedestrians and cyclists, stationary or parked vehicles and collisions arising from side swipes and lane changes.

- Distraction and Fatigue Monitors: Two separately-run projects installing in-cab sensors that detect eye and face movement to monitor drivers' alertness. If signs of distraction or fatigue are recognised an immediate alert is sent to the driver via a vibration in their chair. This will aim to reduce collisions with other road users and provide invaluable data to better understand the issue of fatigue. 
Bus safety can be a criterion for awarding bus contracts to operators and for the allocation of monetary rewards to operators. In London, driver quality monitoring and engineering quality monitoring are already elements of the tender evaluation process. Introducing safety indicators in Quality Incentive Contracts (QIC), however, remains challenging. For example, basing operators' rewards on avoiding near misses could incentivise drivers to stop reporting them, thereby distorting data that could be used for research and prevention. One has to keep in mind the safety culture core elements and the Safe Management System principles mentioned above in Box 12 when choosing performance indicators.

In regions where bus position and speed can be accurately monitored with GPS through fleet management systems, operators and regulators should monitor and reward speed limit compliance at all times. In addition, user feedback should be facilitated by posting a vehicle reference both inside and outside of each public transport vehicle.

\section{Insurance telematics and their impact on behaviour}

Insurance telematics use information collected from a vehicle and its driver so that insurers analyse driving patterns, estimate risk levels and adjust their clients' premiums accordingly. These telematics are also used to establish stipulations in Usage Based Insurance (UBI) policies.

Pay-as-you-drive (PAYD) and pay-how-you-drive (PHYD) are different UBI schemes whose premiums are affected by how much a vehicle is driven or how well. Offering monetary rewards for safer driving behaviours has a positive effect on behaviours, as suggested by Greaves and Fifer (2011), Reagan et al. (2013) and Mortimer et al. (2018). In turn, safer driving is expected to result in a lower number of fatal and serious crashes. UBI solutions may also include an eco-driving and safe driving coaching dimension, delivering targeted advice and providing a further nudge towards safer behaviours.

There are more than 20 million active UBI policies in over 58 different countries. The United States, Italy and the United Kingdom have traditionally been the largest markets for UBIs, with respective penetration rates of $5 \%, 16 \%$ and $3 \%$. Yet, 2018 has seen a surge in UBI policies in China following the passing of new regulations. In Europe, the two fastest growing UBI markets in 2018 were Germany and France (Ptolemus, 2018). Customer demand for UBIs is expected to grow over 140 million globally by 2023 (IMS, 2018a).

Insurance telematics typically include speed, acceleration, braking and cornering, but also road types, driving times and time of day. Different technology solutions exist to support insurance telematics. Some require dedicated devices, often called black boxes or "journey data recorders", to be physically connected to the vehicle's on-board diagnostic (OBD) port, accessing precious vehicle information such as odometer readings. In Australia, an experimental study was launched which uses data from the OBD port to examine the extent to which direct feedback and incentive-based insurance modifies a driver's behaviour (Stevenson et al., 2018).

While access to the OBD port is highly desirable, implementation of the OBD port varies across the automotive industry, and the port is not readily usable in driving conditions on some vehicles. Insurers increasingly rely on location services and motion-sensing capabilities found on smartphones to replace odometer information from OBD connection.

Insurance telematics solutions often rely on smartphones for their mobile connectivity, as well, making dedicated telematics SIM cards redundant to some extent, and leading to significant cost savings. Be it for data collection or for connectivity, smartphones are becoming the central component of insurance telematics. "Smartphone Only" solutions are likely to support much of the growth in the UBI sector in the foreseeable future. Hybrid solutions should, nevertheless, be mentioned, whereby a device is connected both to the OBD port for vehicle dynamics and to a smartphone for the cellular connectivity. 
Smartphones bring the added benefit of dual-side camera equipment, which insurers could use to collect new information on headways in front of the vehicle and on driver visual distractions inside the vehicle. Smartphones could replace dashcam equipment, popular in some countries. Some models also include facial scanners capable of working in the dark. These could potentially be used to monitor signs of driver fatigue, for example in eye lid movements.

Smartphones can detect which vehicle they are travelling in and activate the journey data recorder app through the use of beacons. Beacons are independent battery-powered devices implanted in vehicles. They send a wireless signal to smartphones which allow them to identify vehicles. The coupling of smartphone solutions with such beacons is now at the cutting edge of technology (IMS, 2018b).

While OBD-based telematics solutions used to be leading the market, smartphones offer much cheaper solutions that are quickly gaining as preferred approaches. Smartphone-based solutions also present the unique advantage of portability. Data for a single person can be collected regardless of the vehicle - or even the transport mode - that individual uses. This opens the pathway for new designs in insurance policies for the future, insuring one person's mobility as a whole.

If insurance telematics are proven to improve behaviours, what could regulators and the industry do to support their uptake? All stakeholders should certainly acknowledge that multiple use cases (such as UBI, eco-driving coaching and road user charging) could compete for access to the same OBD port, making a strong case for a wireless alternative to be standardised and developed.

The physical OBD port is certainly well-suited for garage inspections and crash data retrieval during indepth investigations. However, for all other use cases, there is value in defining a standard protocol governing the secure, one-way data broadcast to devices vetted by vehicle occupants. Such a broadcast is already being standardised as part of a cooperative ITS ecosystem. Could UBI smartphone apps simply listen to C-ITS messages as they are broadcast, and successfully identify the insured vehicle's own broadcast? Integration of OBD messages with existing vehicle infotainment Bluetooth connection could be envisaged to support both coaching apps and insurance telematics.

\section{Driver accountability}

Regulators have imposed a number of obligations on drivers of motor vehicles, in line with the risk they pose to third parties. Driver licensing, graduated licensing, health checks, demerit point systems, vehicle registration, insurance obligation and regular technical controls are some of the most widespread obligations on road safety grounds. Will innovative technology and new transport services impact these regulations, often developed decades ago? Potentially so, and reviewing their definition and implementation could deliver significant benefits and cost savings.

\section{Enforcement}

Beyond the regulations themselves, enforcement capability is following a similar trajectory as new digital technologies. Automatic Number Plate Recognition (ANPR) technology facilitates insurance checks on the roadside, and enables segment-based speed controls.

In France, 700000 people drive without insurance and $7 \%$ of all road deaths occur in crashes involving an uninsured vehicle. From 1 January 2019, police checks have been facilitated by a national file containing key information about each person insured. The details include vehicle registration, the name of the insurer, the insurance contract number and its validity period (DSCR, 2018). ANPR will support police checks and aid in detecting uninsured vehicles. The file may also support awareness and information campaigns targeted at owners of uninsured vehicles. 
While ANPR greatly facilitates law enforcement activities, it is vulnerable to the use of fake, damaged or partly concealed number plates. Companies such as Kapsch TrafficCom suggest supplementing license plates with electronic tags, attached to vehicles by the licensing authorities, as part of a system called Electronic Vehicle Registration (Kapsch, n.d.). Such a system, along with the associated enforcement activity, would prevent the use of vehicles that have failed vehicle safety inspections and are thus uninsured. It would also prevent some drivers from purposely avoiding camera-based traffic enforcement.

Applications like Waze enable users to report fixed and mobile speed camera locations, giving users the confidence that speeding will not be enforced outside of these locations, potentially leading users to adopt excessive and dangerous speeds. For this reason, tactical speed enforcement should evolve. Average speed cameras, using automatic number plate recognition at both ends of a road segment, not only cover a greater share of the road network but also prove to be more acceptable in that they allow road users to go over the speed limit for a short period of time when they see fit.

Applying the principles of average speed cameras, also known as section speed enforcement, to the context of toll roads would lead to a situation where tolling and enforcement equipment would be co-located, at access and exit points. This would no doubt raise the question of a possible integration between tolling and speed enforcement systems.

Hit and Run

In Belgium and in the United Kingdom, over $10 \%$ of injury crashes involve a hit and run driver (Kluppels, 2016). The three main causes to this phenomenon are: 1 ) there is some confusion about the legal requirement to stop at a crash scene; 2 ) in particular when the incident is not considered serious enough to report; and 3) some drivers may not be qualified to drive or may not be insured.

However, evidence suggests that the lack of a valid insurance policy is not the main cause for failing to stop at a crash scene. Hit and run events have not decreased at the same rate as the number of uninsured drivers in the past ten years (West, 2016). Only $4 \%$ to $5 \%$ of survey respondents declared they had left the scene of an accident because they were not insured (Hopkins and Chivers, 2016). In Belgium, the share of insured drivers responsible in a hit and run was only 16\% (Kluppels, 2016).

According to Hopkins and Chivers (2016), only $14 \%$ of hit and run drivers claim they would have stopped if they had known they were recorded on CCTVs. The authors claim that the most effective solution to preventing hit and run would be awareness campaigns to warn people of their duty to stop.

Nonetheless, the deployment of effective solutions for tracing hit and run drivers would contribute to preventing such events and making road users feel more accountable. The growing number of video cameras along road networks would contribute to better policing, but there are doubts as to the investment of human resources that would be necessary for the retrieval and analysis of video footage. Video cameras would be most relevant in hit and run investigations if they had ANPR capabilities. For regulators this raises the question of whether they wish to approve the storage of and allow police access to footage from camera equipment originally dedicated to road user charging, low emission zones or traffic surveys.

eCall technology was described earlier. Where a crash triggers the eCall system, emergency services would have a role to play in preventing a driver from leaving the crash scene out of panic or lack of awareness. Emergency services would also receive exact crash location and time stamp, greatly facilitating police investigations, despite the lack of vehicle identification in eCall.

Vulnerable road users (pedestrians, cyclists and powered two-wheeler riders) are particularly overrepresented in hit and run crashes and make up $68 \%$ of hit and run fatalities in the United States (NHTSA, 2017). To detect impacts with vulnerable road users, the question of eCall trigger sensitivity will be raised again, and so will that of EDR trigger. To facilitate police investigations, regulations could impose that EDRs 
retain data on suspected impacts over a relevant period of time to allow police investigators to identify hit and run drivers before crash data is erased.

In the decades ahead, will new requirements be put on EDRs? One could imagine that new data could help forensically trace road users who didn't stop at a crash scene, whether they are crash participants or witnesses. To do so, EDRs could include not only a video feed, but also the digital signatures (MAC addresses) of all nearby wireless devices: connected cars, on-board entertainment systems, smartphones, smartwatches, smart glasses, etc. The technology would be somewhat similar to that which is used in Bluetooth traffic surveys. 


\section{Expanding Cooperative Intelligent Transport Systems}

Cooperative Intelligent Transport Systems, or C-ITS, offer wireless solutions for vehicles to exchange trusted messages and cooperate with other vehicles, road infrastructure and other road users. Those messages are often time-critical and safety-related. Where C-ITS messages are sent and received by vehicles, they are referred to as V2X, or Vehicle to Everything. Messages can be further classified according to road users and road-side units involved: Vehicle to Vehicle (V2V), Vehicle to Pedestrian (V2P) and Infrastructure to Vehicle (I2V) and communications.

C-ITS are a specialisation of common Intelligent Transport Systems (ITS). Known applications of ITS in the transport safety realm include fleet telematics, insurance telematics, alcohol interlock, variable message signs. Whereas some ITS solutions involve wireless communication, they do not generally consist of the cooperative sharing of information by all traffic participants.

C-ITS services will support, but are not limited to autonomous driving. Their benefits will apply to all connected vehicles, regardless of the level of automation. C-ITS services do not by default integrate smartphones and their applications, such as for pedestrians and cyclists, into the V2X ecosystem. In fact, smartphones do not by default fulfil the requirements of ITS stations, such as implementing the required CITS radio access technology with the necessary performance or the required security policies. Retrofitting with C-ITS stations might enable older vehicles to be connected.

In a typical C-ITS ecosystem, a connected vehicle sends and receives messages through its vehicle C-ITS station. A roadside C-ITS station enables infrastructure managers to receive, relay or send messages. A roadside C-ITS station is typically fixed, hosted by a roadside cabinet, traffic signal or other equipment, but can also be mobile, hosted by a maintenance vehicle. C-ITS information is exchanged publicly, thereby increasing the awareness of each entity about the surrounding traffic situation. A central C-ITS station provides up-to-date information to the roadside C-ITS station and collects traffic data from the roadside stations in order to provide traffic management services. Personal C-ITS stations could conceivably be carried by vulnerable road users, such as pedestrians, road workers or cyclists, but as noted earlier, these users should be detected "in real life", rather than connected, in order to not unduly place responsibility for crashes on the most vulnerable road traffic participants.

C-ITS is based on the principle of cooperation, which means using and contributing to the ecosystem in an equal way. The reception of C-ITS information on existing smartphone via non-C-ITS channels might provide benefits to non-connected users, but is not part of the C-ITS context and is prohibited, for example, by the European Union's draft Delegated Act for the deployment and operation of cooperative Intelligent Transport Systems and Services (C-ITS). Therefore, all receiving parties shall also be contributing parties and registered in the security system.

\section{Road safety use case}

Enhancing road safety is often described as the first ambition of C-ITS services. Hundreds of thousands of fatal and serious injuries could be avoided by 2040 in Europe alone thanks to C-ITS technology (5GAA, 2017).The European Union has defined road safety and traffic management application of C-ITS as "Day 1", i.e. priority services (EC, 2019). Table 2 provides an extensive list of C-ITS road-safety related use cases as defined by the European Union.

Some C-ITS services are expected to provide drivers with essential warning signals and efficiently improve road safety. This is the case, for instance, when road users or an infrastructure operator broadcast a warning of adverse conditions ahead, such as slippery roads or sudden fog. Similarly, information about road work and stationary vehicles allows drivers to avoid collisions. In such instances, road users unable of 
receiving low-latency C-ITS messages could receive them through more traditional, higher-latency channels, such as cellular networks, displayed on smartphone navigation apps, for instance.

Table 2. C-ITS safety-related use cases

\begin{tabular}{|c|c|c|}
\hline Service category & Service/use case & $\begin{array}{l}\text { Type of } \\
\text { communication }\end{array}$ \\
\hline Traffic jam & Dangerous end of queue & V2V \\
\hline \multirow{3}{*}{ Stationary vehicle warning } & Stopped vehicle & $\mathrm{V} 2 \mathrm{~V}+\mathrm{I} 2 \mathrm{~V}$ \\
\hline & Broken-down vehicle & $\mathrm{V} 2 \mathrm{~V}+\mathrm{I} 2 \mathrm{~V}$ \\
\hline & Post-crash/Accident zone & $\mathrm{V} 2 \mathrm{~V}+\mathrm{I} 2 \mathrm{~V}$ \\
\hline \multirow{2}{*}{ Special vehicle warning } & Emergency vehicle in operation & V2V \\
\hline & Stationary safeguarding emergency vehicle & V2V \\
\hline \multirow{5}{*}{ Dangerous situation } & Electronic emergency brake light & V2V \\
\hline & Automatic brake intervention & V2V \\
\hline & Reversible occupant restraint system intervention & V2V \\
\hline & Animal or person on the road & $\mathrm{I} 2 \mathrm{~V}$ \\
\hline & Obstacle on the road & $\mathrm{I} 2 \mathrm{~V}$ \\
\hline \multirow{3}{*}{ Adverse weather conditions } & Fog & $\mathrm{V} 2 \mathrm{~V}+\mathrm{I} 2 \mathrm{~V}$ \\
\hline & Precipitation & $\mathrm{V} 2 \mathrm{~V}+\mathrm{I} 2 \mathrm{~V}$ \\
\hline & Traction loss & $\mathrm{V} 2 \mathrm{~V}+\mathrm{I} 2 \mathrm{~V}$ \\
\hline In-vehicle signage & Dynamic speed limit information & $\mathrm{I} 2 \mathrm{~V}$ \\
\hline Road works warning & Road works - mobile & $\mathrm{I} 2 \mathrm{~V}$ \\
\hline
\end{tabular}

Source: adapted from EC (2019).

Traffic safety applications of C-ITS will be somewhat limited by the commercial penetration of connected vehicles. A transition period should be anticipated during which older vehicles and connected vehicles will coexist. This period could last several decades in low- and middle-income countries. In this context, disseminating messages on road hazards to older vehicles directly upstream would require that variable message signs be fitted to the back of more recent vehicles. This thought experiment illustrates the need for regulators to imagine more practical solutions for making C-ITS inclusive. Smartphones and their apps may be such a solution.

It is not difficult to imagine a future where the vast majority of road users receive C-ITS messages on their smartphones (Qualcomm, 2015). Car insurers proposing smartphone-based insurance telematics could consider offering such a solution as part of their apps. It is, however, unclear how to integrate smartphones into the C-ITS ecosystem since they do not - in current European frameworks - qualify as C-ITS stations.

Earlier work by the ITF (2018c) reported a lack of consensus for C-ITS in live safety-critical applications. Connectivity between autonomous vehicles may not be robust enough in times of crisis or in the face of cyberattacks. In other words, autonomous vehicles should, similarly to human drivers, be able to stop within the range of forward vision, as stipulated in Article 13 of the Vienna Convention (UNECE, 1968).

C-ITS nonetheless provides road safety benefits as it increases domain awareness beyond the range of forward vision, which is likely to deliver better safety outcomes. Care should be taken, though, to prevent this technology from having a rebound effect, whereby drivers of connected vehicles drive at higher speeds.

C-ITS messages are of different kinds. Locally, at a range of several hundred metres, short range communication supports the exchange of several types of messages, of which two are particularly relevant in a traffic safety context and are known in Europe as:

- Cooperative Awareness Messages (CAM): proactive messages broadcast every one to ten seconds with general information about vehicle dynamics, including their speed and position 
- Decentralised Environmental Notification Messages (DENM): event-based, time-limited messages triggered under abnormal conditions, for instance when a traffic jam is detected.

The traffic management centre communicates with on-board units not only through roadside units and short range communication technologies, but also through cellular networks.

\section{Competing solutions for short-range communication}

Two main technologies have been developed for C-ITS short-range wireless communications: ITS-G5 and LTE-V2X. ITS-G5 is based on the Wi-Fi 802.11p protocol and designed for unlicensed radio spectrum and shared band use. LTE-V2X is designed for licenced radio spectrum and exclusive band use. ITS-G5, is widely commercially available and the European Commission supports the use of ITS-G5 solutions for road safety C-ITS. The Commission also recognises that technology is changing rapidly and plans for LTE-V2X to access the market in the near future (EC, 2019).

Figure 11. Wireless technologies mobilised for C-ITS services

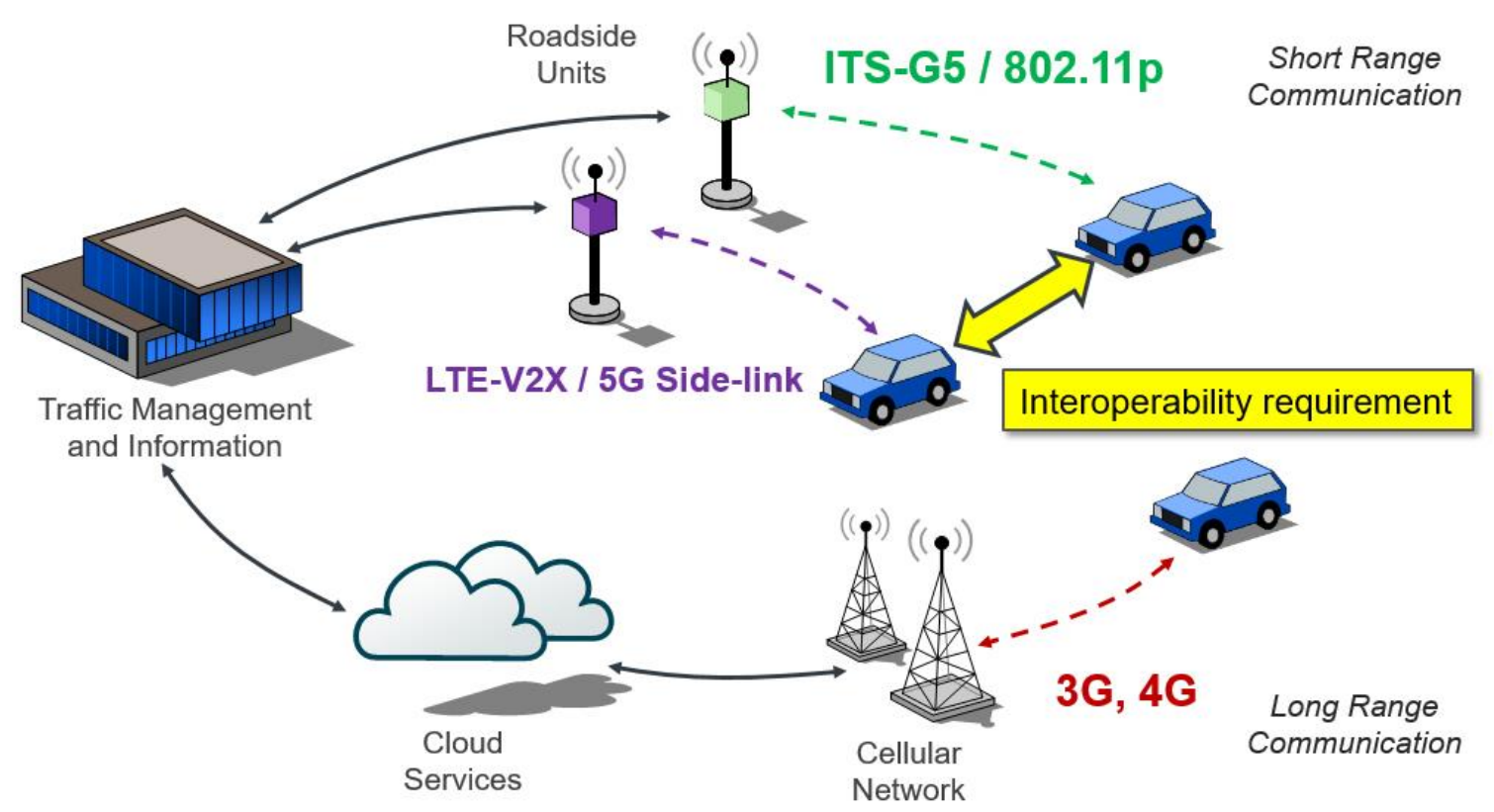

Source: Autotalks, Kapsch, NXP, Cohda Wireless, and Siemens (2018).

The possible coexistence of two wireless technologies is envisioned by the European Commission. It implies the manufacturing of "bilingual" vehicles and roadside units to ensure interoperability. On one hand, this could add resilience to the system. On the other, it could cause channel congestion and generate higher equipment costs for both the automotive industry and infrastructure managers.

\section{Box 13. Coexistence of different standards on the $\mathbf{5 . 9}$ gigahertz band}

In the United States and the European Union, the 5.9 gigahertz $(\mathrm{GHz})$ band is licence exempt and intended for transport safety. In the European Union, the band is a shared space for many technologies, meaning that any technology that would like to operate on this band must take into account any other technology already operating on it. Unlicensed frequency bands are like parks or a public spaces: there are rules to which users must adhere so everybody can use the public space. 
In contrast, licenced band has an owner who has the exclusive right to decide how the band is used and which technology runs there. Technologies for licenced band only need to consider what the band's owner requires of them or are made to operate exclusively on a frequency band. Licenced band is similar to a garden or private space: a set authority establishes the rules that apply there. The owner can be selective and has full authority to decide what may or may not happen in that space.

ITS-G5 is a microwave technology similar to Wi-Fi from the IEEE family of standards. These standards are designed for use on unlicensed frequency bands and sharing with other communication technologies on the same band (other IEEE standards, Bluetooth, etc.). LTE-V2X technologies are derivatives of cellular technologies, which were initially made for licenced-band and exclusive-band use. Without common rules, i.e. mitigation methods, ITS-G5 and LTE-V2X are prone to interfere with each other. LTE-V2X is also prone to interfere with any other communication technology designed for the use on unlicensed bands (eg. in Europe, electronic road charging or the digital tachograph in HGV). A mitigation method is necessary to make co-channel co-existence of LTE-V2X with any other possible technology.

To achieve co-channel co-existence, ITS-G5 and LTE-V2X should not speak at the same moment. One possibility can be a reservation message which includes a rule defining at which intervals messages can be sent. The reservation message asks anybody in the frequency band to give a split second of silence for it to pass. The rule on the interval makes sure that there are always time slots for both (or more) technologies available to broadcast messages. Technology A announces that it would like to transmit a message at a certain moment, the others who receive this message will remain silent for this one moment. The rule on the interval limits the risk of a single entity monopolising the band for an undetermined amout of time and allows others to transmit, as well.

There are several ways this can be technically achieved. However, rules must be established and implemented since there is no authority who decides what technologies may run on the $5.9 \mathrm{GHz}$ band and under which conditions. ITS-G5 "language" could be used for the basic channel coordination and reservation functionalities to avoid jeopardising the functionality of the deployed equipment .

Source: Kapsch.

\section{Protecting vulnerable road users with cooperative systems}

C-ITS have been developed mainly by and for the automotive industry. As a result, C-ITS may be missing out on the opportunity to improve the safety of vulnerable road users (VRUs). Pedestrians, cyclists and powered two-wheeler riders together make up eight out of ten fatalities in cities (Santacreu, 2018a). A majority of experts acknowledged that C-ITS traffic safety applications benefitting VRUs are not as mature as those benefitting car occupants. Applications which require pedestrian smartphones to display alerts may become distractions or give a false sense of protection. Applications which require pedestrians to carry smartphones as signal generator would raise fairness questions. As discussed earlier, the onus should be on the most dangerous traffic participants to detect the more vulnerable. Some sensible C-ITS applications, therefore, include those whereby a roadside observation unit detects and reports unexpected pedestrian or cyclist presence to oncoming traffic.

Similarly, there is a widespread and unchallenged belief that C-ITS services can accurately notify drivers of the presence of VRUs. Problems arise when such a service allows speeding through blind bends and around corners, as shown in Figure 12. Legally, such a service encourages violation of Article 13 of the Vienna Convention on Road Traffic, which specifies that driving decisions must be made "within [the driver's] range of forward vision and short of any foreseeable obstruction" (UNECE, 1968). 
Figure 12. Some C-ITS safety-related use cases
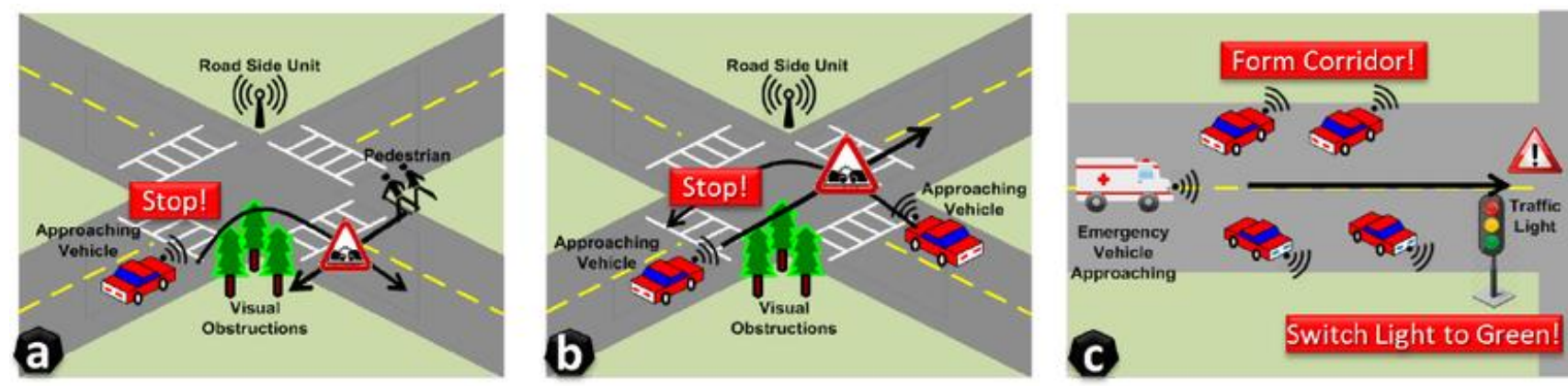

Source: Ben Hamida, Noura and Znaidi (2015).

Transport professionals are actively exploring two solutions to prevent crashes where vulnerable road users are not seen by drivers of larger vehicles, such as buses or trucks. One is the direct vision principle, whereby vehicles should be designed with lower cabs and larger windows so as to keep other road users in the driver's field of vision. Another is through assistance systems that alert the driver when vulnerable road users are in close proximity of the vehicle or when a collision is anticipated.

The two solutions are often in conflict in the sense that one would distract manufacturers and regulators from exploring the other. Nevertheless, they are not mutually exclusive and their combined deployment can be envisaged.

Direct vision design is thought to reduce reaction time in comparison to mirror or camera vision.

Regulations in some countries have limited the overall length of trucks, which resulted in the typical cabover-engine designs that help maximise cargo space. However, the high position of the driver led to an increased blind spot and poor direct visibility around the truck cab. This is a major factor for truck crashes involving vulnerable road users. The number of casualties could be reduced significantly by improving direct vision. Requirements should therefore be introduced to improve the direct vision.

Camera-assisted vision, detection and warning systems are the focus of rapid innovation. The European Commission is proposing that all new trucks and buses be fitted with collision warning signal for pedestrians and cyclists. Questions, however, remain as to how drivers will manage information overload due to false positives or environments with high VRU density. That said, like all systems using data collected by the vehicle for in-vehicle driver assistance, they do not rely on data sharing, and are not further discussed in this report.

Transport for London (TfL, 2018) states that,

Good direct and indirect vision alone will not eliminate all casualties in manoeuvring collisions; the driver must still be looking in the right direction at the right time. Systems that give the driver additional information about the hazards around the bus, or warn of imminent collision, still have an important role to play. How these information and warnings are communicated to the driver is critical to their success and a draft standard accounting for different functionalities, the avoidance of false alarms, and the appropriateness of the human machine interface (HMI) has been developed. 


\section{Box 14. European projects PROSPECT and XCYCLE develop innovative solutions to protect pedestrians and cyclists}

PROSPECT (Proactive Safety for Pedestrian and Cyclists) was a Horizon 2020 funded project which started in May 2015 and ended in October 2018. It was carried on by a variety of partners including OEM and car manufacturers (Continental, Bosh, Daimler, Volvo, Audi, BMW, Toyota), research institutes (BASt, IFSTTAR, TNO, VTI) and universities (Budapest University of Technology and Economics, Chalmers, University of Nottingham, University of Amsterdam).

The European project aimed at improving the effectiveness of vehicles' active safety systems regarding VRUs. The project sought to enhance the quality of crash avoidance technologies with upgraded pedestrian and cyclist detection. To do so, PROSPECT intended to: 1) expand the scope of VRU scenarios addressed; and 2) improve overall system performance. More precisely, PROSPECT targeted five key objectives:

- $\quad$ Better understanding of relevant VRU scenarios (mostly urban)

- Improved VRU sensing and situational analysis

- $\quad$ Advanced HMI and vehicle control strategies

- Four vehicle demonstrators, a mobile driving simulator and a realistic bicycle dummy demonstrator

- $\quad$ Testing in realistic traffic scenarios and user acceptance study.

Another European project, XCYCLE, was a Horizon 2020 EU-funded project which started in June 2015 and ended in October 2018. Running in parallel to the PROSPECT project, it was led by different actors including companies (Volvo, Dynniq, KITE, Jenoptik), research institutes (DLR, VTI), and universities (University of Bologna, University of Leeds, University of Groningen).

XCYCLE sought to contribute to innovative safety measures to reduce the number of crashes between cyclists and motor vehicles. It did so by developing:

- Technologies aimed at improving active and passive detection of cyclists

- $\quad$ Systems informing both drivers and cyclists of a hazard at junctions

- $\quad$ Effective methods of presenting information in vehicles and on-site

- $\quad$ Cooperation systems aimed at reducing collisions with cyclists.

With regards to product outcomes, XCYCLE has developed an in-vehicle detection system with optimized HMI, a system of threat mitigation and risk avoidance by traffic signals, as well as a demonstration bicycle with a cooperative technology.

Unlike PROSPECT, XCYCLE exclusively focuses on cyclist safety. Another main difference with the PROSPECT project lies in the fact XCYCLE installs devices on bike as well as on motor vehicles. This makes bike detection systems more reliable while also allowing for the sending of direct feedback to cyclists. XCYCLE innovations are based on communication and warning systems which could be integrated into a wider cooperative ITS ecosystem. XCYCLE had also developed adaptive traffic light controller and optimal speed advice technology in order to improve cyclist experience and safety by reducing the risk of red light violations and number of stops.

Sources: Gohl et al. (2016), Aparicio (2014), XCYCLE (2018).

Collision warning systems use camera or radar technologies to alert the driver to a potential collision with a pedestrian, cyclist or other vehicle. The intention is that the driver reacts and avoids the collision. 
TRL studied the detection of pedestrians and cyclists near buses and evaluated two detection systems. The first used both radar and camera technologies to detect cyclists undertaking the rear quarter of the bus. The second system used cameras and image processing algorithms to detect both pedestrians and cyclists and had a wider detection area, covering both the nearside and front of the bus. (Edwards et al., 2018)

As part of the Active Safety Collision Warning Project, the state of Washington in the United States recently equipped 38 transit buses with the ROSCO-Mobileye Shield+ System to help drivers avoid and mitigate imminent collisions and protect pedestrians and cyclists. This Collision Avoidance System (CAS) offers a variety of features including pedestrian and cyclist collision warning, forward collision warning, headway monitoring warning, lane departure warning and a speed limit indicator. Dashboard alarms flash when pedestrians enter into the driver's blind spots.

\section{Box 15. Sweden monitors slippery roads with connected vehicles}

In Sweden, connected road vehicles collect data on exceptional weather conditions and loss of traction to improve the planning and execution of road maintenance efforts. With real-time information, the Swedish Transport Administration (Trafikverket) manages the tactical truck deployment for plowing and sanding. The administration will then be able to act quickly and strategically to minimise the period that road surfaces are slippery in cold weather. The Swedish Transport Administration Trafikverket awarded contracts to three different solutions providing friction estimates on a road map every 500 metres. The three solutions rely on different technologies but deliver the same kind of information to the administration:

- $\AA$ F processes data from Volvo vehicles and provides Trafikverket with data on road conditions. All Volvo cars in Sweden fitted with Slippery Road Alert anti-skid technology are involved in the data collection.

- NIRA collects friction data from a taxi fleet and shares the results in real time.

- RoadCloud OY provides road surface friction data to Trafikverket in Gothenburg and Västerås areas. RoadCloud developed a special measurement system that uses an optical sensor and data from the car CAN bus. The measurement system is installed in commercial vehicles, such as taxis, buses and transportation vehicles, operating in Gothenburg and Västerås.

Source: Swedish Transport Administration Trafikverket.

\section{Risk mapping from collaborative intelligence}

Cooperative Awareness Messages (CAMs) are messages exchanged between C-ITS units to create and maintain awareness of each other and to support cooperative applications. CAMs sent by vehicles contain time, position, motion state and activated systems. They also contain attribute information, such as dimensions, vehicle type and role in the road traffic. CAMs should be disseminated between one and ten-times a second, depending on vehicle speed and channel congestion status (ETSI, 2018).

Cooperative awareness messages publicly provide information about vehicle traffic. This information could be collected by roadside units, such as road side cabinets and smart street furniture, but also amateur receivers in roadside properties. This information could later be aggregated and archived to better understand traffic dynamics over space and time.

Services already exist which are sending radio receivers to members of the public in exchange of real-time traffic information via the Internet. MarineTraffic and FlightAware rely on global arrays of receivers to collect detailed positioning data from ships and aircraft (Automatic Identification System and Automatic Dependent Surveillance - Broadcast respectively). A similar business model may develop around road traffic and deliver great benefits to the road safety community seeking to analyse traffic dynamics. This 
technology would become increasingly valuable as and when connected cars reach a greater market penetration, and would compete with video analytics.

While collecting data on hard-braking events is precious, the ability to compute the risk of occurrence is even more so. This involves relating a number of events to a volume of traffic. The relevant denominator in this context, to represent traffic volume, is the number of connected cars passing by a roadside receiver. CITS technology will offer this opportunity through the broadcast of regular CAM messages by all connectedvehicles, even when no particular incident happens.

\section{Railway safety applications at level crossings}

Most fatal crashes in the railway industry occur at railway level crossings. The principle reason for this is that they stand as "uncontrolled" interfaces in an otherwise considerably controlled environment. While the look and feel of level crossings have not changed much in the last few decades, road users regularly find themselves hit by trains at these locations. Whether these accidents are due to deliberate crossing misuse or other causes is unclear.

Current systems could be improved in several ways. First, fixed sensors can detect the presence of road users at level crossings and alert train drivers. The same sensors can also detect a pattern of recurring misuse of the crossing and support countermeasures, like engineering, education or enforcement (UNECE, 2016). Some believe that such sensors could be enabled by combining artificial intelligence with video cameras, infra-red cameras, radar or solid-state LIDAR, the cost of which would be incommensurably less than grade separation.

\section{Box 16. Latvian Railway and Datakom develop smart safety measures for level crossings}

Latvian Railway operates over 3000 kilometres of railway tracks and more than 500 level crossings. The company has started cooperating with the local IT industry to prevent crashes through timely alerts of possible obstacles at level crossings.

In 2018, Latvian Railway participated in the X-Industry Hackathon, organised by the IT Cluster of Latvia. The aim of the hackathon was to bring together various industries with IT experts to develop brand new, custom-made solutions to current problems. In the case of Latvian Railways, this took the shape of a specific solution for railway level crossings.

During the several weeks of the hackathon, Latvian Railway teamed up with Datakom. Together they conceptualised a video analytics system with the ability to follow an object in motion (e.g. vehicles crossing railway tracks at unauthorised times) and warn the infrastructure manager of potential danger. Upon detection of a dangerous situation, the system would immediately broadcast an alert to the railway management centre, to the signalling system, and to the train driver's cabin.

Introduction of this system would decrease the number of casualties on railway crossings. The system is under development and a test-location has been selected.

Source: Latvian Railway.

In addition to technological responses to level crossing crashes, rail traffic control should be able to predict potential crash situations. A typical crash scenario happens at junctions where a passing train may obscure a road user's view of another train arriving from the opposite direction. Once such a situation is predicted:

- visual warnings could be given to users so they do not engage in the crossing after the first train has passed

- C-ITS messages could be generated to the attention of nearby road users. 


\section{Aircraft traffic safety}

The aviation market is growing rapidly. The International Air Transport Association (IATA) forecasts that passenger numbers could double to 8.2 billion per year by 2037. The importance of aviation safety, already a primary concern, will only continue to grow.

According to the International Civil Aviation Organization (ICAO) Aviation Safety Report, there were 50 fatalities (out of 88 accidents) from the scheduled commercial departures in 2017. This level is far lower than for other modes of transport. However, once an aircraft accident occurs, it leads to serious disaster. Constant efforts to ensure aviation safety cannot be overemphasised.

ICAO reported that accidents related to runway and ground safety accounted for more than half of all accidents in 2017. To prevent accidents that could occur on the airport surface, Advanced Surface Movement Guidance and Control System (A-SMGCS) plays an important role in airport operations. ASMGCS is a guidance and control system for ensuring the highest safety level under all weather conditions within the aerodrome visibility operational level. Based on flight schedule, weather information and movement data on the ground, the system provides:

- Surveillance: accurate position of aircrafts, vehicles and obstacles to controller

- Routing: best route for each aircraft and vehicle

- Guidance: guidance necessary for any authorized movement

- Control: resolution when it detects any possibility of incursion.

Incheon International Airport, the largest airport in South Korea, has been operating A-SMGCS at level four for the first time in the world since 2008. The key of A-SMGCS level four is to provide an optimised individual route for each aircraft: from gate departure to runway and from runway to take-off; and from landing to docking at the gate. Moreover, it also controls the aeronautical ground lights to give visual aids to pilots and vehicle drivers to prevent potential conflict with other mobiles. Incheon International Airport is now working on upgrading its A-SMGCS to level five. This will deliver guidance not only to airplanes but to all other airside vehicles as well.

\section{Figure 13. Dashboard of a surface movement control system at Incheon International Airport}

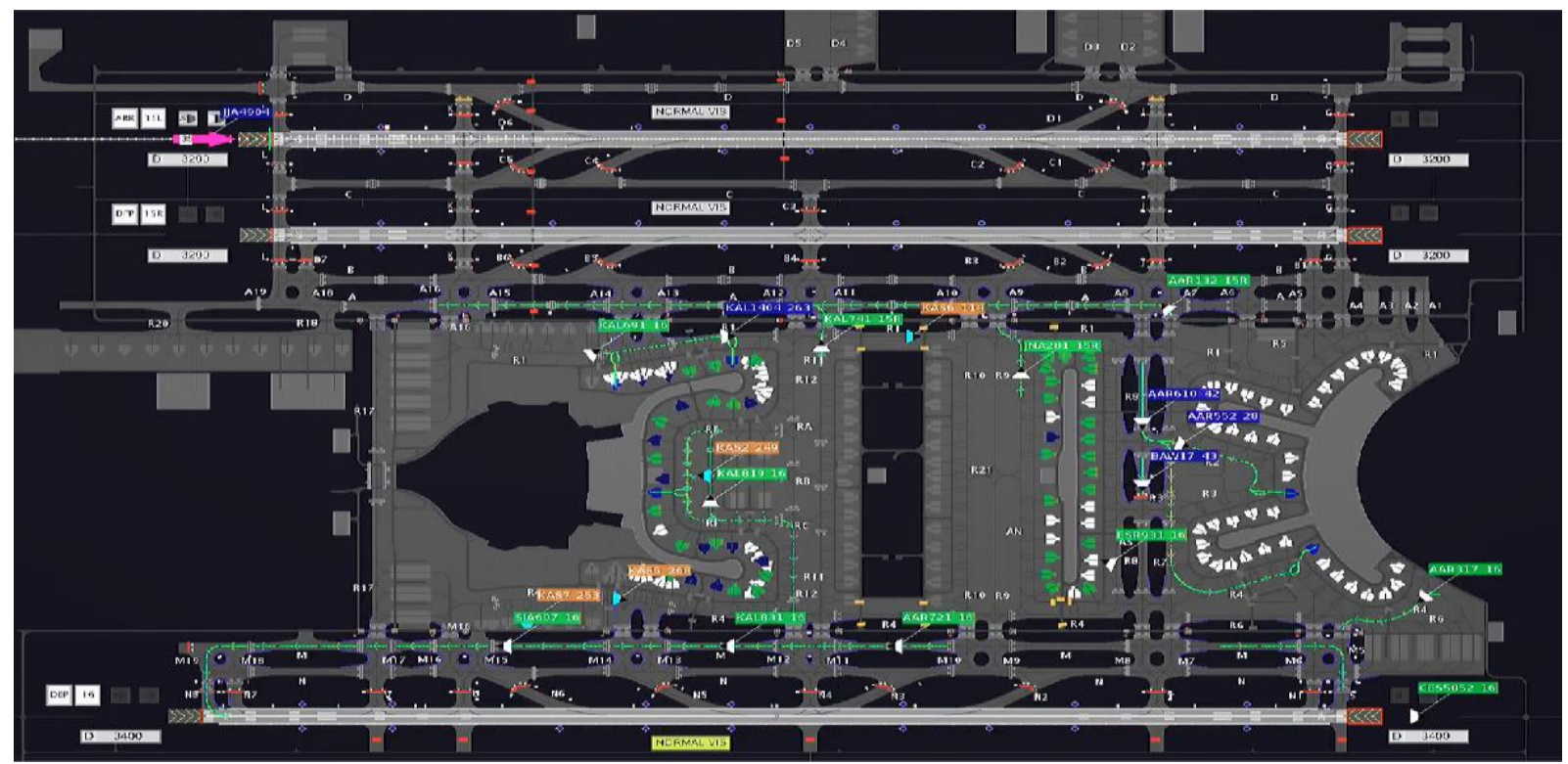

Source: Incheon International Airport. 


\section{Addressing the barriers to data sharing}

This last section is dedicated to addressing the barriers which may prevent the sharing of data for road safety applications. Barriers can be organisational, technical, and are often reinforced by privacy concerns.

As discussed earlier, there would be great value in the transport industry sharing event-based geolocated information for aggregate analysis and mapping. This would include occurrences of active safety system engagement (ABS, ESP and AEB systems in particular) and occurrences of falls automatically detected by micro-mobility devices. The interpretation of such data would require that some form of exposure data was collected from the same vehicles while protecting both commercial interests and road user privacy.

\section{Privacy protection}

The nature of data collection is changing. The access to and use of this data can lead to dangerous outcomes: not least, the unintended and unwanted erosion of privacy rights. Research that contributed to the ITF Corporate Partnership Board report "Big Data and Transport: Understanding and Assessing Options" (ITF, 2015) expressed doubt on whether authorities and regulators - both in and out of the transport sector - have kept pace with the proliferation of new, or newly available, data.

Location data can reveal sensitive information on a person's religion, political affiliation, and health by keeping a log of repeated visits to a place of worship, to political gatherings or to hospitals. Location data should therefore not be linked back to an individual.

However, even anonymised, location data can be used to re-identify an individual, as acknowledged in Article 4 of the European General Data Protection Regulation (GDPR). Indeed, daily routines give away individuals' home and work addresses, tremendously facilitating a re-identification attempt.

This risk of re-identification grows with the number and diversity of exploitable datasets (including social media where people choose to tag their location) and the increasing computing capacity deployed (ultimately with the arrival of quantum computing systems).

\section{De-identification}

Data collectors and processors have at their disposal a multitude of de-identification techniques that range from simple anonymisation to cryptographic protection. Data minimisation is the most important safeguard in protecting personally identifiable information, including for a variety of research purposes and data analysis. The use of strong de-identification techniques, data aggregation and encryption techniques, in particular, are absolutely critical.

If proper de-identification techniques and re-identification risk management procedures are used, reidentification becomes a very difficult task. While there may be a residual risk of re-identification, in the vast majority of cases, de-identification will strongly protect the privacy of individuals when additional safeguards are in place (OIC, 2019).

For the sake of public interest, a trade-off must, therefore, be made between privacy protection and the secondary analysis of location data for transport planning or road safety applications. 


\section{Box 17. Examples of de-identification techniques}

Suppression: removing information that may identify individuals or which, in combination with other information, is reasonably likely to identify an individual.

Rounding: grouping identifiers into categories or ranges. For example, age can be combined and expressed in ranges $(25-35$ years) rather than single years $(27,28)$ or the more detailed date of birth. Extreme values above an upper limit or below a lower limit can be placed in an open-ended range, such as an age value of "less than 15 years" or "more than 80 years", for example.

Perturbation: altering information that is likely to enable the identification of an individual in a small way, such that the aggregate information or information is not significantly affected but the original values cannot be known with certainty. For example, randomly adding or subtracting one to a person's year of birth.

Swapping: swapping information that is likely to enable the identification of an individual for one person with the information for another person with similar characteristics to hide the uniqueness of some information. For example, a person from a particular town in Australia may speak a language that is unique in that town.

Information about that individual's spoken language could be swapped with the spoken language information for another individual with otherwise similar characteristics (based on age, gender, income or other characteristics as appropriate) in an area where the language is more commonly spoken.

Sampling: when very large numbers of records are available, it may be adequate for analysis purposes to release a sample of records, selected through some stated randomised procedure. This creates uncertainty that any particular person is included in the information set.

Encryption or "hashing" of identifiers: information is encrypted or obscured using a scheme that enables accurate analytics to be performed on it, while never revealing the encrypted raw information.

Generating synthetic information: mixing up the elements of an information set-or creating new values based on the original information-so that all of the overall totals, values and patterns of the set are preserved but do not relate to any particular individual.

Source: OIC (2019).

\section{Crash maps}

Road incidents and event data are perhaps the easiest of the applications to deal with, given that only one location is needed and the risk of discovery of individual's identity and trip-making patterns is eliminated. Unlike other sequential location-based data, incident data concerns traffic or road events that have occurred but are not necessarily connected to an individual's movements (ITF, 2016c).

More than ten countries already publish online maps of crash locations, according to a survey conducted among countries represented in the ITF IRTAD group (Owen, 2018). Maps include exact crash location and severity. However, countries have adopted very different approaches to the disclosure of further crash information. Some provide very little information, not even the date and time. Others choose to disclose casualty age, nationality and blood alcohol concentration.

\section{Opening up video analytics}

Besides floating vehicle data, video analysis from a surveillance camera network should be mined for close call events, and support a proactive approach to road safety interventions. This raises several questions:

- Will this be compatible with privacy protection principles? We certainly recommend that video images collected for close call analysis purposes are degraded in such a way that neither faces nor number plates are recognisable. Faces could be replaced by avatars so that head orientation data is 
not lost. In addition, one could discard video sequences where no close call is observed. The detection of close calls could even be done locally at the level of the sensor itself, so that irrelevant video sequences are not sent to the cloud in the first place.

- Will local authorities have the capacity to analyse and take action on the basis of video images? There is probably value in sharing an open video feed for researchers and members of the public to run their own surrogate safety analysis. A platform called Archive of Many Outdoor Scenes (AMOS) stores and archives images from public webcam feeds (Hipp et al., 2013; Hipp et al., 2016). This platform, however, is not designed to store video feeds at a frame rate that would support transport safety analysis.

\section{Box 18. Archive of Many Outdoor Scenes}

The Archive of Many Outdoor Scenes (AMOS) has archived over one billion images of outdoor environments from 30000 public webcams since 2006. Webcam images are captured at the rate of one image per camera per hour and given a time stamp. Many of the locations have seen improvements in the built environment, including complete streets, bike share start-ups or walking school bus programmes. AMOS is able to document and allow quantification of population behaviour changes (such as mode shift) following the changes to the built environment.

Source: Hipp et al. (2013).

\section{Tools, models and partnerships for sharing data}

Making sense of Big Data requires skills and resources. Most safety relevant data could be wasted for lack of analytical resources. This is where common data standards and referencing systems have a major role to play.

Figure 14. Map-matched GPS points generate data on roadway traffic speeds

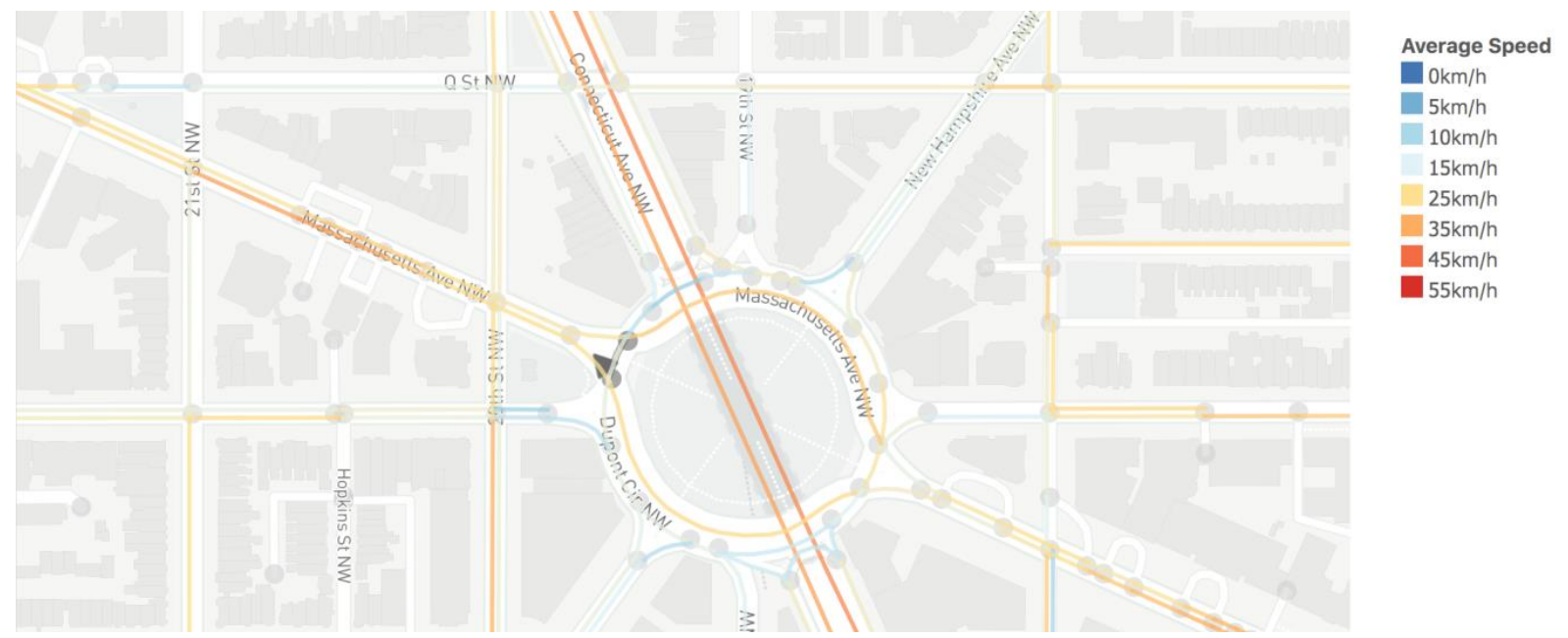

Source: SharedStreets (n.d.).

Launched in 2018, SharedStreets is an open data standard and digital platform for mapping city streets and sharing associated data. It is capable of collecting and making sense of information from various transport operators, even though they all use different base maps (NACTO, 2018; NACTO and OTP, 2018). The protocol is based on an open-source linear referencing system. It ensures accurate location data while being forgiving of different geospatial referencing systems embedded in commercially-sensitive base maps. The platform is used to collect locally binned and aggregated information on hard braking events, pick-up 
and drop-off events, speed, etc., thus enabling public-private collaboration to manage streets, reduce traffic deaths, and prepare cities for emerging changes in mobility services.

Already operating in over 30 cities around the world, the SharedStreets platform and will provide city leaders with new, far-reaching instruments for managing transportation networks. Initial SharedStreets implementations cover:

- producing a universal framework for sharing curb-side passenger pick-up and drop-off counts

- $\quad$ producing the first-ever freely available global data set of vehicle driving speeds.

By introducing a universal language for digitally describing every aspect of city streets, the platform overcomes long-standing legal, regulatory and technological barriers between the public and private sectors' ad hoc and disparate transportation data sources.

\section{Box 19. HERE's Open Location Platform}

Real-time sensor data from vehicles by multiple automakers is already collected by HERE, a map services company. Their Open Location Platform absorbs this information, which can be as simple as location, heading, and speed. Feedback from the use of hazard lights and fog lights, emergency brakes or electronic stability control, or even from cameras built into the cars can all be blended into the Open Location Platform's database.

The platform offers map-matching solutions to convert raw GPS traces to a coherent network representation. Data is also anonymised and aggregated. The Open Location Platform helps actors of the mobility eco-system merge various data sources to develop new products (such as a navigation map for cyclists) and monetise their locationbased data (such as GPS traces and pothole information). The company provides access through the platform to a repository of location data, which includes traffic and weather.

Sources: Davies (2018), HERE (n.d.).

A number of companies have committed to using SharedStreets to share data with cities. In Sept 2018, Ford Motor Company, Uber and Lyft pledged to share some of their road traffic data using the SharedStreets framework.

New models of public-private partnership involving data-sharing

In Data-Driven Transport Policy, the ITF (2016c) dedicates a chapter to new data sharing models and partnerships. Those new models may be necessary to leverage both public and private benefits.

Under existing data ownership rules a significant amount of the actionable data pertaining to road safety, traffic management and travel behaviour is held by the private sector. A growing movement is underway, however, to re-evaluate the legal framework of personal data ownership - with the EU's General Data Protection Regulation being the most broad-ranging example to date. It embodies many of the key elements of Sandy Pentland's "New Deal on Data" - notably that individuals have a right to possess their data, that individuals should have full control over the use of theur data and that people should have the right to dispose or distribute their personal data as they see fit (Hardjono, Shrier and Pentland, 2016). Should data ownership rules change along the lines of "A New Deal on Data", individuals would retain ownership and control use of this data. Under both data ownership frameworks, public authorities will likely continue to be mandated to provide essential services. In this context, much as public authorities have coercive ability to require access to personal data (e.g. on property ownership, personal revenue, criminal records), there may be scope to define public-access data sets that are built on aggregated personal location and trajectory data. 
These data could relate to traffic flows, crash locations and causes (as reported by embarked-vehicle ITS systems), as well as crowd density, location and movement data. This would entail a move away from the supplier-client relationship that some authorities have with data collectors. Recent moves by some data collectors to share their data with public authorities (e.g. Uber in the Boston area) show that more creative partnerships can be developed that enable both the private sector to innovate and the public sector to carry out its mandates. However, work will be required to define the scope and scale of data access by public authorities and, in particular, to ensure that the collection of such data is in line with public mandates.

A range of solutions, either open source or commercial (Box 19), are being developed to collect, harmonise and aggregate mobility data. We encourage all stakeholders to make road safety relevant data freely available through such platforms. 


\section{Bibliography}

5GAA (2017), "An Assessment of LTE-V2X (PC5) and 802.11p Direct Communications Technologies for Improved Road Safety in the EU", White Papers, 5G Automotive Association, http://5gaa.org/news/anassessment-of-Ite-v2x-pc5-and-802-11p-direct-communications-technologies-for-improved-road-safety-inthe-eu/ (accessed on 20 March 2019).

Adminaite, D. et al. (2017), "Tapping the Potential for Reducing Work-Related Road Deaths and Injuries", European Transport Safety Council, Brussels, https://etsc.eu/wp-content/uploads/PIN FLASH33-final.pdf (accessed on 20 March 2019).

Adminaite, D. et al. (2018), "An Overview of Road Death Data Collection in the EU", European Transport Safety Council, Brussels, https://etsc.eu/wp-content/uploads/PIN-FLASH-35-final.pdf (accessed on 20 March 2019).

Ahangari, H., C. Atkinson-Palombo, N. Garrick (2017), "Automobile-dependency as a barrier to vision zero, evidence from the states in the USA", Accident Analysis and Prevention, Vol. 107, pp. 77-85, https://doi.org/10.1016/j.aap.2017.07.012.

Aldred, R. and S. Crosweller (2015), "Investigating the rates and impacts of near misses and related incidents among UK cyclists", Journal of Transport and Health, Vol. 2/3, pp. 379-393, https://doi.org/10.1016/j.jth.2015.05.006.

Aldred, R. (2018), "Inequalities in self-report road injury risk in Britain: A new analysis of National Travel Survey data, focusing on pedestrian injuries", Journal of Transport and Health, Vol. 9, pp. 96-104, https://doi.org/10.1016/j.jth.2018.03.006.

Aparicio, A. (2014), "PROSPECT PROactive Safety for Pedestrians and CyclisTs", presentation by Applus IDIADA, the PROSPECT project received funding from the European Commission's Innovation and Networks Executive Agency, under the frame of Horizon 2020 programme, with grant agreement no 634149, available under "PROSPECT-General Presentation" at www.prospect-project.eu/download/ (accessed on 20 March 2019).

Ariel, B. et al. (2016), "'Contagious accountability': A Global Multisite Randomized Controlled Trial on the Effect of Police Body-Worn Cameras on Citizens' Complaints Against the Police", Criminal Justice and Behavior, Vol. 44/2, pp. 293-316, Sage Publications Inc, https://doi.org/10.1177/0093854816668218.

Austin City Council (2016), "Vision Zero Action Plan 2016-2018", https://austintexas.gov/sites/default/files/ files/Imagine Austin/VisionZero/ActionPlan 5.19.16adoption.pdf (accessed on 20 March 2019).

Autotalks, Kapsch, NXP, Cohda Wireless, and Siemens (2018), "ITS-G5 Technology - A Fact Sheet", https://ec.europa.eu/info/law/better-regulation/feedback/18167/attachment/090166e5c12a3443 en (accessed on 20 March 2019).

Barrios, J., Y. Hochberg and L. Yi (2018), "The Cost of Convenience: Ridesharing and Traffic Fatalities", New Working Paper Series, No. 27, University of Chicago Booth School of Business, Chicago, https://research. chicagobooth.edu/-/media/research/stigler/pdfs/workingpapers/27thecostofconvenience.pdf (accessed on 20 March 2019).

Barsi, A. and C. Heipke (2004), "Artificial Neural Networks for the Detection of Road Junctions in Aerial Images", The International Archives of the Photogrammetry, Remote Sensing and Spatial Information Sciences, Vol. 34, Part 3/W8, pp. 113-118, http://taurus.gg.bg.ut.ee/kalle r/KAUGSEIRE2/2007/ Publikatsioonid/Barsi\&Heipke2004.pdf (accessed on 20 March 2019).

Ben Hamida, E., H. Noura and W. Znaidi (2015), "Security of Cooperative Intelligent Transport Systems: 
Standards, threats analysis and cryptographic countermeasures", Electronics, Vol. 4, pp. 380-423, doi: $10.3390 /$ electronics 4030380 .

Berneking, M. et al. (2018), "The risk of fatigue and sleepiness in the ridesharing industry: An American Academy of Sleep Medicine position statement", Journal of Clinical Sleep Medicine, Vol. 14/4, pp. 683-685, http://doi.org/10.5664/jcsm.7072.

Bloomberg Philanthropies (n.d.), "Data for Health", Bloomberg Philanthropies, www.bloomberg.org/ program/public-health/data-health/\#overview (accessed on 12 February 2019).

BLS (2018), "Contingent and Alternative Employment Arrangements - May 2017", New Release: Bureau of Labor Statistics, Department of Labor (United States), Washington, DC, www.bls.gov/news.release/ pdf/conemp.pdf (accessed on 20 March 2019).

Bolderdijk, J. et al. (2011), "Effects of Pay-As-You-Drive vehicle insurance on young drivers' speed choice: Results of a Dutch field experiment", Accident Analysis and Prevention, Vol. 43/3, pp. 1181-1186, http://doi.org/10.1016/j.aap.2010.12.032.

Bosco, E. (2017), "Map of the Month: Vision Zero Boston", Data-Smart City Solutions, https://datasmart.ash.harvard.edu/news/article/map-of-the-month-vision-zero-boston-1081 (accessed on 20 March 2019).

Brazil, N. and D. Kirk (2016), "Uber and metropolitan traffic fatalities in the United States", American Journal of Epidemiology, Vol. 184/3, pp. 192-198, https://doi.org/10.1093/aje/kww062.

Burke, K. (2018), "Al is my co-pilot: How DRIVE IX helps humans stay focused", Nvidia blog, https://blogs.nvidia.com/blog/2018/06/27/drive-ix-helps-humans-stay-focused/ (accessed on 23 January 2019).

Carnis, L. (2017), Les Politiques d'Automatisation des Contrôles de Vitesse: Entre Logiques Institutionnelles, Formes Organisationnelles et Contraintes Opérationnelles, IFSTTAR.

Chang, A. and K. Wilson (2018), SAE Standardization Activities in Road Safety Data, SAE International (unpublished).

Chang, A., N. Saunier and A. Laureshyn (2017), "Proactive Methods for Road Safety Analysis", SAE Technical Paper, WP-0005, SAE International.

Chhatkuli, S. et al. (2017), "Automated road markings extraction from mobile vehicle borne laser scanning data", International Symposium on Remote Sensing, Nagoya, to request publication please see: www.researchgate. net/publication/317041214 AUTOMATED ROAD MARKINGS EXTRACTION FROM MOBI LE_VEHICLE_BORNE_LASER_SCANNING_DATA (accessed on 23 January 2019).

Cho, H. et al. (2017), "Novel heart rate detection method using UWB impulse radar", Journal of Signal Processing Systems, Vol. 87/2, pp. 229-239, https://doi.org/10.1007/s11265-016-1177-7.

Christie, N. and H. Ward (2018), "The emerging issues for management of occupational road risk in a changing economy: A survey of gig economy drivers, riders and their managers", UCL Centre for Transport Studies, www.ucl.ac.uk/drupal/site news/sites/news/files/a-survey-of-gig-economy-drivers-riders-andtheir-managers 1.pdf (accessed on 20 March 2019).

College of Paramedics (2014), "National Ambulance Patient Record Standards in partnership with the College of Paramedics", College of Paramedics, www.collegeofparamedics.co.uk/news/national-ambulancepatient-record-standards-in-partnership-with-the-college (accessed on 19 February 2019). 
Conderino, S. et al. (2017), "Linkage of traffic crash and hospitalization records with limited identifiers for enhanced public health surveillance", Accident Analysis and Prevention, Vol. 101, pp. 117-123, http://doi.org/10.1016/j.aap.2017.02.011.

Daud, M. et al. (2018), "Using real driving data for transportation policy: A proof of concept", report prepared for Dash labs. Inc, Robert F. Wagner Graduate School of Public Service, New York, https://wagner.nyu.edu/files/about/DASH Report v3.pdf (accessed on 20 March 2019).

Davies, C. (2018), "HERE's crowdsourced road safety data just got its first customer", Slash Gear, www.slashgear.com/here-safety-services-suite-bmw-2018-deal-03513495/ (accessed 12 February 2019).

Deloitte (2017), "Global mobile consumer trends, 2nd edition: Mobile continues its global reach into all aspects of consumers' lives", www2.deloitte.com/global/en/pages/technology-media-andtelecommunications/articles/gx-global-mobile-consumer-trends.html (accessed on 20 March 2019).

DfT (2017), "British social attitudes survey 2016: Public attitudes towards transport, Statistical Release, Department for Transport, London, https://assets.publishing.service.gov.uk/government/uploads/system/ uploads/attachment data/file/640297/british-social-attitudes-survey-2016.pdf (accessed on 20 March 2019).

DfT (2018), "Reported road casualties in Great Britain: 2017 annual report", Statistical Release, Department for Transport, London, https://assets.publishing.service.gov.uk/government/uploads/system/ uploads/attachment data/file/744077/reported-road-casualties-annual-report-2017.pdf (accessed on 20 March 2019).

DS Automobiles (2017), "DS Driver Attention Monitoring", DS Automobiles Official Youtube Channel, www. youtube.com/watch?v=oVxRCUhbAMI\&feature=youtu.be (accessed on 20 January 2019).

DSCR (2018), "Création du fichier des véhicules assurés (FVA) pour lutter contre la conduite sans assurance en facilitant les contrôles des forces de l'ordre ", Direction de la sécurité et de la circulation routières, www.securite-routiere.gouv.fr/medias/espace-presse/publications-presse/creation-du-fichier-des-vehiculesassures-fva-pour-lutter-contre-la-conduite-sans-assurance-en-facilitant-les-controles-des-forces-de-l-ordre (accessed on 11 February 2019).

Dupont, E. and JC. Meunier (2017), "Lésions subies suite à l'implication dans un accident de la circulation : quelles sont les spécificités des cyclistes et des motards? " (Injuries suffered as the result of being involved in a road accident: what are the specific details regarding cyclists and motorcyclists?), Research report no. 2017-R-08-FR, VIAS Institute, Brussels, www.vias.be/publications/Wat $\% 20$ is $\% 20$ specifiek\%20voor\% 20fietsers\%20en\%20motorfietsers\%20bij\%20de\%20verwondingen\%20opgelopen $\% 20$ bij\%20een \%20verke ersongeval/Lesions subies suite a I implication dans un accident de la circulation.pdf (accessed on 20 March 2019).

EarthNow (2018), "EarthNow to deliver real-time video via large satellite constellation", https://earthnow.com/ (accessed on 11 February 2019).

EC (2009), VERONICA - II Final Report, European Commission, Brussels, https://ec.europa.eu/transport/ road safety/sites/roadsafety/files/pdf/projects sources/veronica2 final report.pdf (accessed on 20 March 2019).

EC (2015a), "Commission looks at benefits of Event Data Recorders", Road Safety Newsletter, No. 17, European Commission, Brussels, http://media.wix.com/ugd/fcfed0 b1ffa574d7624a56aa25caa2740 aa1b7.pdf (accessed on 20 March 2019).

EC (2015b), "eCall in all new cars from April 2018", European Commission, https://ec.europa.eu/digitalsingle-market/en/news/ecall-all-new-cars-april-2018 (accessed on 19 January 2019). 
EC (2017), "Road Safety: Encouraging results in 2016 call for continued efforts to save lives on EU roads", European Commission, Press release, http://europa.eu/rapid/press-release IP-17-674 en.htm\# ftn1 (accessed on 20 March 2019).

EC (2018a), "Proposal for a Regulation of the European Parliament and of the Council on type-approval requirements for motor vehicles and their trailers, and systems, components and separate technical units intended for such vehicles, as regards their general safety and the protection of vehicle occupants and vulnerable road users, amending Regulation (EU) 2018/... and repealing Regulations (EC) No 78/2009, (EC) No 79/2009 and (EC) No 661/2009", European Commission, Brussels, https://eur-lex.europa.eu/ resource.html?uri=cellar:f7e29905-59b7-11e8-ab41-01aa75ed71a1.0003.02/DOC 1\&format=PDF (accessed on 20 March 2019).

EC (2018b), "Communication from the Commission to the European Parliament, the Council, The European Economic and Social Committee and the Committee of the Regions: Europe On The Move - Sustainable Mobility for Europe: safe, connected, and clean", European Commission, Brussels, https://eurlex.europa.eu/legal-content/EN/TXT/?uri=COM:2018:293:FIN (accessed on 11 February 2019).

EC (2019), "Commission Delegated Regulation (EU) .../... of XXX Supplementing Directive 2010/40/EU of the European Parliament and of the Council with regard to the Deployment and Operational Use of Cooperative Intelligent Transport Systems" (Draft), European Commission, Brussels, https://ec.europa.eu/info/law/betterregulation/initiative/2045/publication/346736/attachment/090166e5c03ed6f6 en (accessed on 20 March 2019).

Edwards, A. et al. (2018), Analysis of Bus Collisions and Identification of Countermeasures, report prepared for Transport for London, Transport Research Laboratory, Berks, https://trl.co.uk/reports/analysis-buscollisions-and-identification-countermeasures (accessed on 20 March 2019).

EP (2014), European Parliament, www.europarl.europa.eu/RegData/etudes/STUD/2014/529071/ IPOL STU\%282014\%29529071 EN.pdf (accessed on 20 March 2019).

EP (2015), "Automatic emergency call devices in all new car models from spring 2018", European Parliament, Press release, www.europarl.europa.eu/news/en/press-room/20150424IPR45714/automaticemergency-call-devices-in-all-new-car-models-from-spring-2018 (accessed on 19 January 2019).

EP (2018), "Saving lives: eCall mandatory in new car models from this week", European Parliament, Press release, www.europarl.europa.eu/news/en/press-room/20180326IPR00510/saving-lives-ecall-mandatoryin-new-car-models-from-this-week (accessed on 19 January 2019).

ETSI (2018), "Intelligent Transport Systems (ITS); Vehicular Communications; Basic Set of Applications; Part 2: Specification of Cooperative Awareness Basic Service", European Standard, Draft ETSI EN 302 637-2, V1.4.0, https://www.etsi.org/deliver/etsi en/302600 302699/30263702/01.04.00 20/ en 30263702v010400a.pdf (accessed on 20 March 2019).

Fawcett, L. et al. (2017), "A novel Bayesian hierarchical model for road safety hotspot prediction", Accident Analysis and Prevention, Vol. 99/A, pp. 262-271, https://doi.org/10.1016/j.aap.2016.11.021.

Filippi, A. et al. (2018), "Ready to roll: Why 802.11p beats LTE and 5G for V2x", White Paper, NXP Semiconductors, Cohda Wireless, and Siemens, https://assets.new.siemens.com/siemens/assets/ public.1510309207.ab5935c545ee430a94910921b8ec75f3c17bab6c.its-g5-ready-to-roll-en.pdf.

Fishman, E. and P. Schepers (2018), "The Safety of Bike Share Systems", ITF Discussion Papers, International Transport Forum, Paris, https://www.itf-oecd.org/safety-bike-share-systems. 
Ford Smart Mobility (2018), City Data Solutions, Ford of Europe and PRISM International, http://citydatareport.fordmedia.eu/ (accessed on 25 January 2019).

Fu, T., L. Miranda-Moreno and N. Saunier (2018), "A novel framework to evaluate pedestrian safety at nonsignalized locations", Accident Analysis and Prevention, Vol. 111, pp. 23-33, https://doi.org/10.1016/ j.aap.2017.11.015.

Gao, F. et al. (2014), "Moving vehicle information extraction from single-pass WorldView-2 imagery based on ERGAS-SNS Analysis", Remote Sensing, Vol. 6, No. 7, pp. 6500-6523, http://doi.org/10.3390/ rs6076500. Gohl, I. et al. (2016), "Car-to-cyclist accidents from the car driver's point of view", presented at the International Cycling Safety Conference, Bologna, http://www.prospect-project.eu/download//accessed on 19 January 2019).

Götschi, T. et al. (2018), "Towards a comprehensive safety evaluation of cycling infrastructure including objective and subjective measures", Journal of Transport and Health, Vol. 8, pp. 44-54, https://doi.org/ 10.1016/j.jth.2017.12.003.

Grass, M. (2018), "San Diego looks at 'geofencing' to slow e-scooters", Route Fifty, https://www.routefifty.com/smart-cities/2018/10/san-diego-geofencing--e-scooters/152292/ (accessed on 22 March 2019).

Greaves, S. and S. Fifer (2011), Analysis of a financial incentive to encourage safer driving practices, Working Paper ITLS-WP-11-18, Institute of Transport and Logistics Studies, Sydney, https://ses.library. usyd.edu.au/bitstream/2123/19234/1/ITLS-WP-11-18.pdf (accessed on 19 January 2019).

GRR (2018), "ATO has been operated with ETCS during passenger service for the first time", Global Railway Review, www.globalrailwayreview.com/news/67562/ato-etcs-passenger-service/ (accessed on 19 January 2019.

GSA (2016), "Satellite navigation at core of future connected car systems", European Global Navigation Satellite Systems Agency, www.gsa.europa.eu/newsroom/news/satellite-navigation-core-future-connectedcar-systems (accessed 25 January 2019).

GSA (2018), SARHA project: Sensor-Augmented EGNOS/Galileo Receiver for Handheld Applications in urban and indoor environments, GNSS Project Portfolio, www.gsa.europa.eu/sensor-augmentedegnosgalileo-receiver-handheld-applications-urban-and-indoor-environments (accessed 25 January 2019).

GSMA (2018), The Mobile Economy 2018, GSMA Intelligence, London, https://www.gsmaintelligence.com/ research/2018/02/the-mobile-economy-2018/660/ (accessed on 19 January 2019).

Guilbot, M., L. Vaslin, and E. Arregle (2018), "Véhicule connecté, communicant, automatisé et protection des données à caractère personnel des usagers", IFSTTAR presentation at Les Rencontres de la Mobilité Intelligente Conference held on 25 January 2018, Paris.

Hardjono, T., D. Shrier and A. Pentland (eds.). (2016), Trust:: Data: A New Framework for Identity and Data Sharing, CreateSpace Independent Publishing Platform, http://www.visionaryfuture.com/trust-data.html (accessed on 23 April 2019).

Here (n.d.), Here Open Location Platform, Website Homepage, https://openlocation.here.com/ (accessed on 12 February 2019).

Hipp, J. et al. (2013), "Emerging technologies: Webcams and crowd-sourcing to identify active transportation", American Journal of Preventive Medicine, Vol. 44/1, pp. 96-97, https://doi.org/10.1016/ j.amepre.2012.09.051. 
Hipp, J. et al. (2016), "Webcams, crowdsourcing, and enhanced crosswalks: Developing a novel method to analyze active transportation", Frontiers in Public Health, Vol. 4/97, http://doi.org/10.3389/fpubh.

2016.00097.

Hopkins, M. and S. Chivers (2016), "A national survey: Why do drivers fail to stop and report an accident? Interim findings", research commissioned by MIB, University of Leicester, UK, https://www.mib.org.uk/ media-centre/news/2016/april/new-research-shows-why-drivers-hit-and-run/ (accessed on 19 January 2019).

Hydén, C. (1987), The Development of a Method for Traffic Safety Evaluation: the Swedish Traffic Conflict Technique, Lund University, retrieved from UC Berkeley Transportation Library (Accession No. 00422429).

Hynd, D. et al. (2016), Benefit and Feasibility of a Range of New Technologies and Unregulated Measures in the Fields of Vehicle Occupant Safety and Protection of Vulnerable Road Users, report prepared for European Commission, Transport Research Laboratory, http://dx.doi.org/10.2769/497485.

Hynd, D., and M. McCarthy (2014), Study on the Benefits Resulting from the Installation of Event Data Recorders, report prepared for the European Commission, Transport Research Laboratory, http://dx.doi.org/10.2832/66709.

IDTP (2017), "Africa rising in Kigali, Rwanda", IDTP Sustainable Transport Magazine, Issue 28, www.itdp.org/2017/03/06/africa-rising-in-kigali/ (accessed 20 February 2019).

IIHS-HLDI (2019a), "Distracted driving: Cellphone manipulations up 57 percent over prior survey", Insurance Institute for Highway Safety and the Highway Loss Data Institute Status Report, Vol. 54/1, www.iihs.org/iihs/sr/statusreport/article/54/1/1 (accessed on 30 January 2019).

IIHS-HLDI (2019b), "Modest use for iPhone 'Do Not Disturb' blocker", Insurance Institute for Highway Safety and the Highway Loss Data Institute Status Report, Vol. 54/1, www.iihs.org/iihs/sr/ statusreport/article/54/1/3 (accessed 30 January 2019).

Immergis (2016), Support and consulting services for national road assets management, Professional Service brochure, http://immergis.fr/brochures/EN/Immergis ProfessionalServices EN 2016.pdf.

IMS (2018a), "Mobile telematics: The game changer for insurance telematics", Featured Insights Blog, Intelligent Mechatronic Systems, https://www.intellimec.com/insights/mobile-telematics-apps-autoinsurance (accessed on 8 February 2019).

IMS (2018b), "Comparing Mobile Apps, Hybrid Bluetooth and OBD-II: Data Collection Options", Intelligent Mechatronic Systems White Paper, https://www.intellimec.com/ubiwhitepaper.

IMS (n.d.), "How to collect driving data: Powering your insurance telematics programs", Featured Insights Blog, Intelligent Mechatronic Systems, https://www. intellimec.com/insights/how-to-collect-telematicsdriving-data (accessed 11 February 2019).

iRAP (2018), "Vaccines for Roads", 4th edition, International Road Assessment Program, https://www.vaccinesforroads.org/about/ (accessed 11 February 2019).

ITF (2015), "Big Data and Transport: Understanding and Assessing Options", International Transport Forum Policy Papers, No. 8, OECD Publishing, Paris, https://doi.org/10.1787/5jlwvzdb6r47-en.

ITF (2016a), Zero Road Deaths and Serious Injuries: Leading a Paradigm Shift to a Safe System, OECD Publishing, Paris, https://doi.org/10.1787/9789282108055-en.

ITF (2016b), Road Safety Annual Report 2016, OECD Publishing, Paris, https://doi.org/10.1787/irtad-2016en. 
ITF (2016c), Data-Driven Transport Policy, Corporate Partnership Board Report, International Transport Forum, Paris, https://www.itf-oecd.org/sites/default/files/docs/data-driven-transport-policy.pdf.

ITF (2016d), App-Based Ride and Taxi Services: Principles for Regulation, Corporate Partnership Board Report, International Transport Forum, Paris, https://www.itf-oecd.org/sites/default/files/docs/app-ridetaxi-regulation.pdf.

ITF (2017), Road Safety Annual Report 2017, OECD Publishing, Paris, https://doi.org/10.1787/irtad-2017en.

ITF (2018a), Speed and Crash Risk, OECD Publishing, Paris, www.itf-oecd.org/speed-crash-risk.

ITF (2018b), Safety Management Systems: Summary and Conclusions, ITF Roundtable Reports, No. 172, OECD Publishing, Paris, https://doi.org/10.1787/d5fda9c4-en.

ITF (2018c), Safer Roads with Automated Vehicles?, Corporate Partnership Board Report, International Transport Forum, Paris, www.itf-oecd.org/sites/default/files/docs/safer-roads-automated-vehicles.pdf.

ITF (2018d), Cooperative Mobility Systems and Automated Driving: Summary and Conclusions, ITF Roundtable Reports, No. 167, OECD Publishing, Paris, https://doi.org/10.1787/5ca48905-en.

ITF (2018e), Cycling Safety: Summary and Conclusions, ITF Roundtable Reports, No. 168, OECD Publishing, Paris, https://doi.org/10.1787/7f4da6f4-en.

ITF (2019a), Commercial Vehicle On-Board Safety Systems: Summary and Conclusion, ITF Roundtable Reports, No. 163, OECD Publishing, Paris, https://doi.org/10.1787/896c39ab-en.

ITF (2019b), Governing Transport in the Algorithmic Age, Corporate Partnership Board Report, International Transport Forum, Paris.

ITF/OECD (2011), Reporting on Serious Road Traffic Casualties, IRTAD research report, International Transport Forum, OECD Publishing, Paris, https://www.itf-oecd.org/reporting-serious-road-trafficcasualties.

Jo, J. et al. (2011), "Vision-based method for detecting driver drowsiness and distraction in driver monitoring system", Optical Engineering, Vol. 50/12, http://doi.org/10.1117/1.3657506.

Kamaluddin, N.A, et al. (2018), "Self-reporting traffic crashes - a systematic literature review", European Transport Research Review, Vol. 10/26, https://doi.org/10.1186/s12544-018-0301-0.

Kapsch (n.d.), Electronic Vehicle Registration, Kapsch TrafficCom, Vienna, https://www.kapsch.net/ktc/downloads/datasheets/video-sensor/Kapsch-KTC-DS-VDR.pdf?lang=en-US (accessed 30 January 2019).

Kluppels, L. (2016), "Beyond shame and guilt: What's inside a hit and run accident", Belgian Road Safety Institute, http://traffic-psychology-international.eu/wp-content/uploads/2016/01/Beyond-shame-andguilt.pdf (accessed on 22 March 2019).

Knowles, J. et al. (2009), Collisions Involving Pedal Cyclists on Britain's Roads: Establishing the Causes, prepared for RSRSD Department for Transport (United Kingdom), Transport Research Laboratory, www.worthingrevolutions.org.uk/sites/worthingrevolutions.org.uk/files/PPR445.pdf (accessed on 30 January 2019).

Kontschieder, P. (2017), "Releasing the World's Largest Street-level Imagery Dataset for Teaching Machines to See", https://blog.mapillary.com/product/2017/05/03/mapillary-vistas-dataset.html (accessed on 30 January 2019). 
Krambeck, H., S. Job and S. Sultan (2018), "Better Crash Data Can Improve Road Safety", Connections Transport Global Practice, World Bank, http://pubdocs.worldbank.org/en/577571537471058626/ TransportGlobalPractice-Note-September-web.pdf (accessed on 22 March 2019).

Lagarde, J-P. (2017), "Les entreprises dominent toujours le marché automobile", L'Opinion, https://www.lopinion.fr/edition/economie/entreprises-dominent-toujours-marche-automobile-124751 (accessed on 22 March 2019).

Larsen, S.Ø., A.B. Salberg and L. Eikvil (2013), "Automatic system for operational traffic monitoring using very-high-resolution satellite imagery", International Journal of Remote Sensing, Vol. 34, pp. 4850-4870, http://doi.org/10.1080/01431161.2013.782708.

Laureshyn, A. et al. (2017), "In search of the severity dimension of traffic events: Extended Delta-V as a traffic conflict indicator", Accident Analysis and Prevention, Vol. 98, pp. 46-56, https://doi.org/10.1016/ j.aap.2016.09.026.

Leitloff, J., S. Hinz and U. Stilla (2010), "Vehicle detection in very high resolution satellite images of city areas", IEEE Transactions on Geoscience and Remote Sensing, Vol. 48, pp. 2795-2806, http://doi.org/ 10.1109/TGRS.2010.2043109.

Li, S. and P. McAleese (2018), "City Verve and See.Sense understanding the invisible cyclist", presentation delivered at the $4^{\text {th }}$ meeting of the ITF Safer City Streets network in Manchester, 21 Nov 2018, https://www.itf-oecd.org/crowdsourced-sensor-data-helping-improve-cyclist-safety.

Litman, T. (2016), The Hidden Traffic Safety Solution: Public Transportation, American Public Transportation Association, Washington, DC, https://trid.trb.org/view/1422707 (accessed on 20 March 2019).

Litman, T. (2019), "Toward more comprehensive evaluation of traffic risks and safety strategies", Research in Transportation Business and Management, Vol. 29, pp. 127-135, https://doi.org/10.1016/j.rtbm. 2019.01.003.

London Assembly Transport Committee (2017), Driven to Distraction Making London's Buses Safer, Greater London Authority, London, https://www.london.gov.uk/sites/default/files/driven-to-distraction-final.pdf (accessed on 20 March 2019).

Madsen, T. et al. (2017), "Mobile application for naturalistic walking/cycling data collection", Horizon 2020 EC Project, InDeV. Aalborg, Denmark: Aalborg University, https://www.forskningsdatabasen.dk/en/catalog/2396706030 (accessed on 19 March 2019).

Mortimer, D. et al. (2018), "The effect of 'smart' financial incentives on driving behaviour of novice drivers", Accident Analysis and Prevention, Vol. 119, pp. 68-79, https://doi.org/10.1016/j.aap.2018.06.014.

NACTO (2018), "Ford Motor Co., Uber and Lyft announce agreement to share data through new platform that gives cities and mobility companies new tools to manage congestion, cut greenhouse gases and reduce crashes", National Association of City Transportation Officials, https://nacto.org/2018/09/26/ford-uber-lyftshare-data-through-sharedstreets-platform/ (accessed on 30 January 2019).

NACTO and OTP (2018), "National Association Of City Transportation Officials and the Open Transport Partnership launch transportation data standard and platform, building foundation for public-private partnerships in the digital age", National Association of City Transportation Officials and Open Transport Partnership, http://sharedstreets.io/public/SharedStreets Release.pdf.

NEMSIS (n.d.) "History of NEMSIS", NEMSIS Official Website, https://nemsis.org/what-is-nemsis/history-ofnemsis/ (accessed on 19 February). 
Neteera Technologies (2018), "Neteera Company Movie 2018", Neteera Technologies Youtube Channel https://youtu.be/3IuplLHIJFQ (accessed on 25 January 2019).

NHTSA (2005), "Event Data Recorder: Overview", National Highway Traffic Safety Administration, Washington, DC, www.nhtsa.gov/research-data/event-data-recorder\#overview-10516 (accessed on 21 January 2019).

NHTSA (2017), "Fatal Accident Reporting System: Analytical User's Manual 1975-2016", US Department of Transportation.

North Wales Police (2016), "Drivers to be prosecuted using dash cam evidence", www.northwales.police.uk/news-and-appeals/drivers-to-be-prosecuted-using-dash-cam-evidence (accessed on 01 February 2019).

Nowakowski, C. et al. (2015), "Development of California regulations to govern testing and operation of automated driving systems", Transportation Research Record, Vol. 2489/1, pp. 137-144, http://doi.org/ $\underline{10.3141 / 2489-16}$.

NYC DOT (2017), "Data matching:Linkage of traffic crash and hospitalization records", presentation given at the second meeting of the Safer City Streets Network, Brussels, December 2017, https://www.itf-oecd.org/ node/21281.

NYPTA (2017), "NYPTA White Paper on Transportation Network Company (TNC) Issues", White Paper, New York Public Transit Association, https://transittraininginstitute.org/images/NYPTA TNC issues White Paper.pdf (accessed on 11 February 2019).

OIC (2019), "Privacy and de-identification", Office of the Information Commissioner, Queensland, https://www.oic.qld.gov.au/guidelines/for-government/guidelines-privacy-principles/applying-the-privacyprinciples/privacy-and-de-identification (accessed on 11 February 2019).

Owen, R. (2018), "Crash data location and mapping", Presentation at the April 2018 meeting of the Transport Research Committee of the Intenational Transport Forum, Paris.

Owens, J. et al. (2018), Crash Risk of Cell Phone Use While Driving: A Case-Crossover Analysis of Naturalistic Driving Data, AAA Foundation for Traffic Safety, Washington, DC, https://aaafoundation.org/ wp-content/uploads/2018/01/CellPhoneCrashRisk FINAL.pdf (accessed on 01 February 2019).

PBOT (2019), 2018 E-Scooter Findings Report, Portland Bureau of Transportation, Portland, https://www.portlandoregon.gov/transportation/78431 (accessed on 08 February 2019).

Perez, K. et al. (2016), Practical Guidelines for the Registration and Monitoring of Serious Traffic Injuries, D7. 1 of the H2020 Project Safetycube, Loughborough University, Loughborough, https://dspace.lboro.ac.uk/2134/23760.

Perez, K. et al. (2018), "Implications of estimating road traffic serious injuries from hospital data", Accident Analysis and Prevention, https://doi.org/10.1016/j.aap.2018.04.005.

Polders, E. and T. Brijs (2018), How to Analyse Accident Causation? A Handbook with Focus on Vulnerable Road Users, Horizon 2020 EC Project, InDeV, Hasselt University, Hasselt, www.indev-project.eu/InDeV/EN/ Handbook/Handbook-InDeV.html.

Porter, A. et al. (2018), "The digital ambulance: Electronic patient clinical records in prehospital emergency care", BMJ Open, Vol. 8.

Ptolemus (2018), "The state of Usage-Based Insurance (UBI) today", Ptolemus Consulting Group, www.ptolemus.com/content/uploads/2018/07/2018-UBI-Infographic.png (accessed on 11 February 2019). 
Qualcomm (2015), "How Snapdragon and Honda are working to save lives with smartphones", Qualcomm OnQ Blog, www.qualcomm.com/news/onq/2015/06/16/how-snapdragon-and-honda-are-working-savelives-smartphones (accessed on 15 February 2019).

Reagan, I. et al. (2013), "The effects of external motivation and real-time automated feedback on speeding behavior in a naturalistic setting", Human Factors, Vol. 55/1, pp. 218-30, http://doi.org/10.1177/0018720812447812.

Reason, J. (1997), Managing the Risks of Organizational Accidents, Ashgate Publishing.

Rezapour, M., S. Wulff and K. Ksaibati (2018), "Effectiveness of enforcement resources in the highway patrol in reducing fatality rates", IATSS Research, Vol. 42/4, pp. 259-264, https://doi.org/10.1016/ j.iatssr.2018.04.001.

Road Safety GB (2018), "Wales: Operation Snap is extended nationwide", http://roadsafetygb.org.uk/news/ wales-operation-snap-is-extended-nationwide/ (accessed on 01 February 2019).

SAE (2018), "Surface transportation recommended practice: Taxonomy and definitions for terms related to shared mobility and enabling technologies", Product code J3163_201809, SAE International, https://doi.org/10.4271/J3163 201809.

Santacreu, A. (2018a), "Safer City Streets Global Benchmarking for Urban Road Safety", International Transport Forum Working Document, OECD Publishing, Paris, www.itfoecd.org/sites/default/files/docs/safer-city-streets-global-benchmarking-urban-road-safety.pdf.

SharedStreets (n.d.), "National Association of City Transportation Officials and the Open Transport Partnership launch transportation data standard and platform, building foundation for public-private partnerships in the digital age", Press Release, https://sharedstreets.io/public/ SharedStreets Release.pdf (accessed on 11 February 2019).

Siemens (n.d.), Internet of Trains 2.0: Everything you Wanted to Know About Digital Services in Rail Industry, publication available upon request at https://new.siemens.com/global/en/products/mobility/railsolutions/services/digital-services/railigent/internet-of-trains-qa.html.

Stevenson, M. et al. (2018), "The effects of feedback and incentive-based insurance on driving behaviours: study approach and protocols", Injury Prevention, Vol. 24/1, pp. 89-93, http://dx.doi.org/10.1136/ injuryprev-2016-042280.

Stipancic, J. et al. (2018), "Surrogate safety and network screening: Modelling crash frequency using GPS travel data and latent Gaussian Spatial Models", Accident Analysis and Prevention, Vol. 120, pp. 174-187, https://doi.org/10.1016/j.aap.2018.07.013.

Strauss, J. et al. (2017), "Cyclist deceleration rate as surrogate safety measure in Montreal using smartphone GPS data", Accident Analysis and Prevention, Vol. 99/A, pp. 287-296, https://doi.org/ 10.1016/j.aap.2016.11.019.

Sun, T. (2018), "Lime scooters in Santa Monica bring equity, access and smart mobility", LimeCities, https://www.li.me/blog/lime-scooters-in-santa-monica-bring-equity-access-smart-mobility (accessed on 11 February 2019).

Tagesspiegel (2018), "Radmesser Project", https://interaktiv.tagesspiegel.de/radmesser/kapitel7.html (accessed on 31 January 2019).

Taub, E. (2017), "Sleepy behind the wheel? Some cars can tell", The New York Times, www.nytimes.com/2017/03/16/automobiles/wheels/drowsy-driving-technology.html (accessed 30 January 2019). 
Technavio (2017), Global Automotive Event Data Recorder Market: 2017-2021, Technavio, publication available upon request at https://www.technavio.com/report/global-automotive-electronics-globalautomotive-event-data-recorder-market-2017-2021 (accessed on 30 January 2019).

TfL (2017a), "TfL response to GLA Transport Committee Report on Bus Safety", Transport for London, www.london.gov.uk/sites/default/files/tfl response to bus safety report.pdf.

TfL (2017b), "Update on Bus Safety Programme: Transport London response to London Assembly Transport Committee Report", Transport for London, https://www.london.gov.uk/moderngov/documents/s70257/ 12c\%20-\%20Appendix \%202\%20Response $\% 20$ to\%20bus\%20report $\% 20-\% 20$ Safety $\% 20$ Programme $\%$ 20Update\%20FINAL.pdf.

TfL (2018), "Bus Safety Standards: Executive Summary", Transport for London, https://trl.co.uk/sites/default/files/articles/Bus\%20Safety\%20Standard\%20-\%20Executive\%20Summary\% 20-\%20TRL-TfL.pdf.

Uber (2017), "City of Chicago Ordinance: Operating hours cap", Uber website, www.uber.com/drive/ chicago/resources/driving-hour-limits// (accessed on 14 February 2019).

UNECE (1968), Convention on Road Traffic, United Nations Economic Commission for Europe: Inland Transport Committee, www.unece.org/fileadmin/DAM/trans/conventn/crt1968e.pdf.

UNECE (2016), Assessment of Safety at Level Crossings in UNECE Member Countries and Other Selected Countries and Strategic Framework for Improving Safety at Level Crossings, United Nations Economic Commission for Europe: Inland Transport Committee, https://www.unece.org/trans/roadsafe/ wp12017.html (accessed on 14 February 2019).

Ursachi, G. et al. (2018), "Driving while impaired by alcohol: An analysis of drink-drivers involved in UK collisions", https://www.researchgate.net/publication/324835697 Driving while impaired by alcohol An analysis of drink-drivers involved in UK collisions (accessed on 12 April 2019).

Vaa, T., T. Assum and R. Elvik (2014), "Driver support systems: Estimating road safety effects at varying levels of implementation", Institute of Transport Economics, Oslo, http://doi.org/10.13140/2.1.2904.0327.

Vicente, J. et al. (2016), "Drowsiness detection using heart rate variability", Medical and Biological Engineering and Computing, Vol. 54/6, pp. 927-937, http://doi.org/10.1007/s11517-015-1448-7.

Vision Zero Network (2018a), "Core Elements for Vision Zero Communities", Case Study, https://visionzeronetwork.org/resources/ (accessed on 20 March 2019).

Vision Zero Network (2018b), "Vision Zero Equity Strategies for Practitioners", Case Study, https://visionzeronetwork.org/resources/ (accessed on 20 March 2019).

WB (2017), The High Toll of Traffic Injuries: Unacceptable and Preventable, The World Bank, Washington DC, https://openknowledge.worldbank.org/handle/10986/29129.

Welle, B. et al. (2018), Sustainable and Safe: A Vision and Guidance for Zero Road Deaths, World Resource Institute, Washington, DC, www.wri.org/publication/sustainable-and-safe-vision-and-guidance-zero-roaddeaths (accessed on 14 February 2019).

West, A. (2016), "The psychology of 'hit and run'", Chief Executive's Blog, MIB, www.mib.org.uk/ about-mib/chief-executives-blog/the-psychology-of-hit-and-run/ (accessed on 14 February 2019).

WHO (2018), Global Status Report on Road Safety 2018, World Health Organization, Geneva, www.who.int/violence injury prevention/road safety status/2018/en/ (accessed on 20 March 2019). 
XCycle (2018), "The XCycle innovations", Bologna University, Bologna, https://site.unibo.it/xcycle/en/ project-material.

Yin, L. et al. (2015), "Re-Identification Risk versus Data Utility for Aggregated Mobility Research Using Mobile Phone Location Data", PLoS ONE, Vol. 10/10, https://doi.org/10.1371/journal.pone.0140589. 


\section{EInternational Transport Forum}

\section{New Directions for Data-Driven Transport Safety}

This report explores how seamless data collection, analysis and sharing can unlock innovations in transport safety. Most interventions to improve transport safety are reactions to incidents. Connected vehicles, smartphone apps, ubiquitous sensors, data sharing and machine learning make proactive transport safety interventions possible and prevent crashes before they happen. Drawing on the Safe System approach, this report examines how transport stakeholders can make better decisions by using more relevant and timely data. 